\title{
A framework for device interaction in a network of things
}

\author{
Pablo César Calcina Ccori
}

\author{
THESIS PRESENTED \\ TO THE \\ Institute of MATHEMATiCS AND StAtistics \\ OF THE \\ University of SÃo Paulo \\ TO \\ OBTAIN THE TITLE \\ OF \\ Doctor of Philosophy
}

\author{
Program: Computer Science \\ Advisor: Prof. Dr. Laisa Caroline Costa de Biase \\ Co-advisor: Prof. Dr. Flávio Soares Correa da Silva
}

This study was financed in part by the

Coordenação de Aperfeiçoamento de Pessoal de Nível Superior - Brasil

(CAPES) - Finance Code 001

São Paulo, March, 2020 


\section{A framework for device interaction in a network of things}

This version of the thesis contains the corrections and modifications suggested by the Judging Committee during the defense of the original version of this work, in July 8th, 2020. A copy of the original version is available in the Institute of Mathematics and Statistics of the University of São Paulo.

Judging Committee:

- Prof. Dr. Flávio Soares Corrêa da Silva (advisor) - IME-USP

- Prof. Dr. Jan Rabaey - UC Berkeley

- Prof. Dr. Roseli de Deus Lopes - EP-USP

- Prof. Dr. José de Jesus Pérez Alcazár - EACH-USP

- Prof. Dr. Daniel Macedo Batista - IME-USP 


\section{Acknowledgments}

I had the rare honor of having three advisors. Three highly talented professors to whom I am very grateful. This thesis was inspired by the insightful work from Dr. Laisa Costa, who patiently accompanied this work from its inception. Her advice and opportune feedback were essential to conduct the present thesis until a satisfactory conclusion. Whenever I was in doubt in the capricious course of the research path, I could always rely on the accurate advice from my second advisor, Prof. Flávio Corrêa da Silva. After long sessions of conversation accompanied by a cup of good coffee, Prof. Corrêa da Silva offered the wise words I needed. My third advisor, Prof. Marcelo Zuffo, was undoubtedly the cornerstone of this cooperation. As the leader of the Interdisciplinary Center of Interactive Technologies (CITI), he is responsible for the development of cutting-edge technology in Brazil, of which this thesis is a humble sample.

Proving that the whole if greater than the sum of the parts, the Swarm team at CITI has produced a tremendous work during these years, in the form of scientific articles, system design, software architecture, application development and abundant documentation. This thesis has directly benefited from that development and I would like to express my gratitude to all the talented people that participated in the Swarm team. Mr. Geovane Fedrecheski provided the official SwarmBroker implementation and the initial version of the SwarmLib, on top of which I implemented the framework. Mr. Carlos Laschi actively collaborated in the definition of the Geolocation Ontology for the Swarm that is presented in this work. Mr. Gabriel Duarte and Mr. Phillipe Rangel participated in the definition of the economic model for the Swarm, also presented in this thesis. Mr. Guilherme C. Marques and Matheus Guinezi contributed with the initial version of the SwarmAssistant, which is the basis of the case study used in this work. Also, Dr. Samira Afzal kindly helped with the revision of this document.

This work was partially supported by Associação do Laboratório de Sistemas Integráveis Tecnológico (LSI-TEC) and Coordenação de Aperfeiçoamento de Pessoal de Nível Superior (CAPES). 


\section{Abstract}

\section{CALCINA-CCORI, Pablo C. A framework for device interaction in a network of things.}

2020. 103 p. Thesis (Doctorate) - Institute of Mathematics and Statistics, University of São Paulo, São Paulo, 2020.

As devices in the IoT are increasing in number and capabilities, there is an opportunity of creating networks of smart devices that go beyond the current cloud-centric model of data-gathering and actuation. The Swarm project provides a middleware to create a bio-inspired distributed and organic network of heterogeneous devices.

Under the context of the Swarm project, in this thesis, we aim to create a framework for the interaction of devices, consisting of registration in the network, discovery, composition, and mediation of services. Using semantics as a driving technique, we aim to create a communication framework that facilitates the development of IoT applications in the Swarm, as a first step towards constructing a smart self-organizing network for the future IoT.

The proposed framework aims to overcome the problems of interoperability and composition by adapting lightweight open standards with a service-oriented architecture and novel composition and mediation mechanisms. To illustrate the use of our framework, we implemented a use case based on the recruiting of services for a surveillance system.

The significant contributions of this thesis can be summarized as: an architecture and implementation for device interaction in the IoT, a lightweight model for semantic service description and semantic querying, a ranking algorithm for service selection in an economy-based IoT network, an ontology for IoT services, and a declarative composition and mediation. To evaluate our work, we used two methods. First, we performed a quantitative comparison between an implementation with and without the use of our framework, then, we conduct a qualitative comparison of features offered by our framework with other similar works.

Keywords: Internet of Things, device interaction, Swarm. 


\section{Resumo}

CCORI, P. C. C. Um arcabouço para interação de dispositivos em uma rede de coisas. 2020. 103 f. Tese (Doutorado) - Instituto de Matemática e Estatística, Universidade de São Paulo, São Paulo, 2010.

À medida que os dispositivos na Internet das Coisas estão crescendo em número e em capacidades, surge a oportunidade de criar redes de dispositivos inteligentes que vão além do modelo atual de coleta de dados e atuação, centrado na nuvem. O projeto Swarm provê um middleware para a criação de redes bioinspiradas de dispositivos heterogêneos.

No contexto do projeto Swarm, nesta tese temos como objetivo criar um arcabouço para a interação de dispositivos, que consiste no registro na rede, descoberta, composição e mediação de serviços.

Usando semântica como técnica direcionadora, temos como objetivos a criação de um arcabouço de comunicação que facilita o desenvolvimento de aplicações para IoT no Swarm, como um primeiro passo para a construção de uma rede auto-organizável para a IoT do futuro.

O arcabouço proposto tem como objetivo atacar os problemas de interoperabilidade e composição, adotando padrões leves com uma arquitetura orientada a serviços e mecanismos novos de composição e mediação. A fim de ilustrar o uso do nosso arcabouço, implementamos um caso de uso baseado no recrutamento de serviços para um sistema de vigilância.

As principais contribuições desta tese podem ser resumidas em: uma arquitetura e implementação para interação de dispositivos na IoT, um modelo leve para a descrição semântica de serviços e consultas, um algoritmo de ranqueamento para seleção de serviços em uma rede IoT baseada em princípios econômicos, uma ontologia para serviços para IoT, e um modelo declarativo para a composição e mediação de serviços.

A fim de avaliar o nosso trabalho, utilizamos dois métodos para avaliar o arcabouço. Primeiro fazemos uma comparação quantitativa entre a implementação do caso de uso com e sem o uso do arcabouço. Também, fazemos uma comparação qualitativa das características oferecidas por nosso arcabouço com outros trabalhos similares.

Palavras-chave: Internet das coisas, interação de dispositivos, Swarm. 


\section{Contents}

$\begin{array}{ll}\text { List of Abbreviations } & \text { xi }\end{array}$

List of Figures $\quad$ xiii

$\begin{array}{ll}\text { List of Tables } & \text { XV }\end{array}$

1 Introduction $\quad 1$

1.1 Challenges ........................... 3

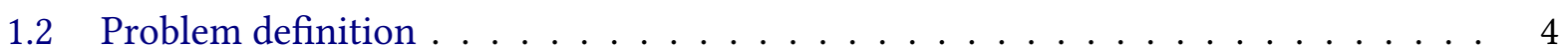

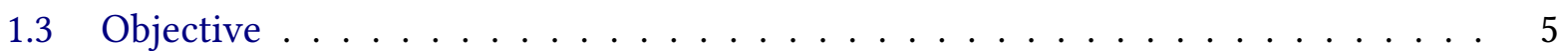

1.4 Hypothesis . . . . . . . . . . . . . . . . . . . . 5

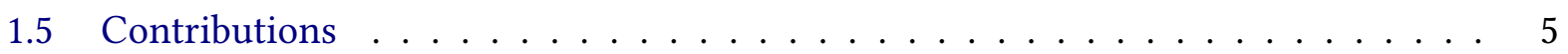

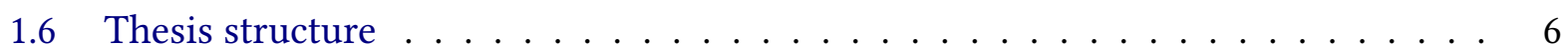

$\begin{array}{lll}2 & \text { Basic concepts and technologies } & 7\end{array}$

2.1 The Swarm . . . . . . . . . . . . . . . . . . . . . 7

2.1 .1 The Swarm project . . . . . . . . . . . . . . 7

2.1 .2 Swarm Broker . . . . . . . . . . . . . . . . . . . . . . 10

2.1.3 Swarm economic model . . . . . . . . . . . . . . . . . 11

2.1 .4 CoAP-HTTP proxy. . . . . . . . . . . . . . . . 12

2.1.5 Swarm minimum broker. . . . . . . . . . . . . . . . . 13

2.1.6 Access control model . . . . . . . . . . . . . . . . . . . . . . 13

2.2 Related visions . . . . . . . . . . . . . . . . . . . . . . . . . 15

2.3 Other related frameworks . . . . . . . . . . . . . . . . . . . . 17

$2.3 .1 \quad$ OWL-S . . . . . . . . . . . . . . . . . . . 17

$2.3 .2 \quad \mathrm{WSMO} \ldots \ldots \ldots \ldots \ldots \ldots \ldots$

$2.3 .3 \quad$ Hydra . . . . . . . . . . . . . . . . . . 18

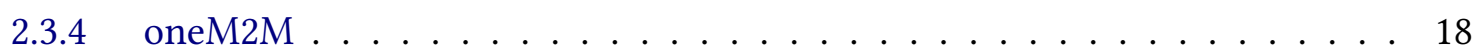

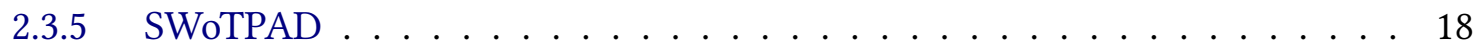

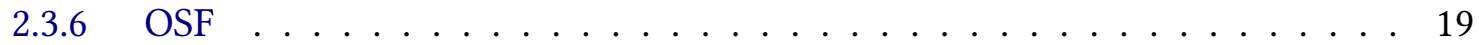

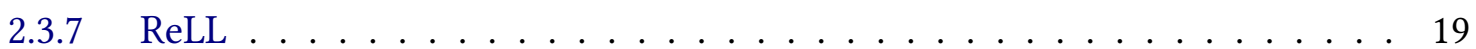

3 Framework definition $\quad 21$

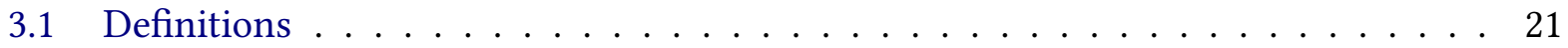




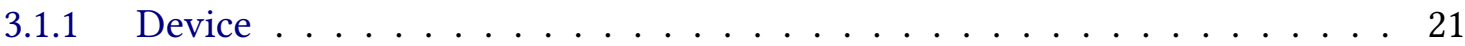

3.1 .2 Network . . . . . . . . . . . . . . . . . . . 22

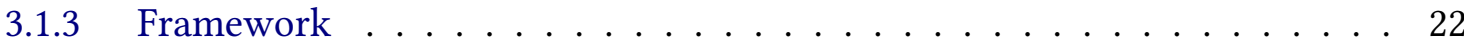

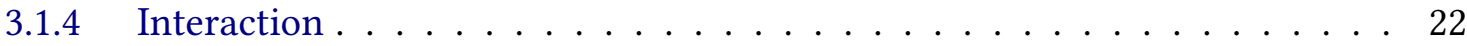

3.2 Main components . . . . . . . . . . . . . . . . . . . . . 24

3.2.1 Service description . . . . . . . . . . . . . . . . . 24

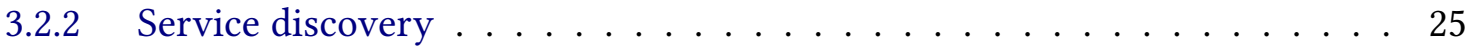

3.2 .3 Semantic query description $\ldots \ldots \ldots \ldots \ldots \ldots$

3.2 .4 Code generator . . . . . . . . . . . . . . . . . 25

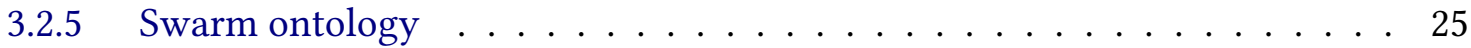

3.2 .6 Semantic registry . . . . . . . . . . . . . . . . . 25

3.2 .7 Mediator . . . . . . . . . . . . . . . . . 25

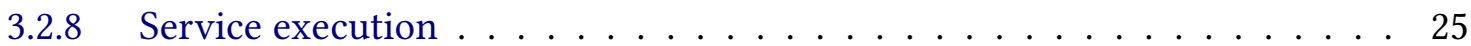

3.2 .9 Service composition $\ldots \ldots \ldots \ldots \ldots \ldots$

3.2 .10 Score computation $\ldots \ldots \ldots \ldots \ldots$

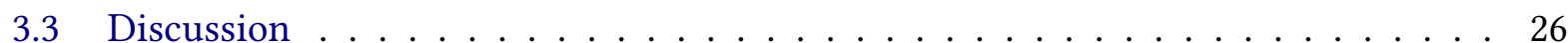

4 Framework architecture $\quad 29$

4.1 Service description model . . . . . . . . . . . . . . . . . . . . 29

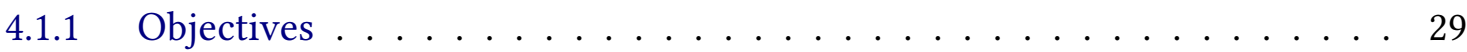

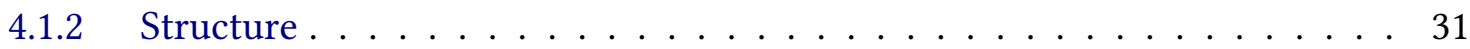

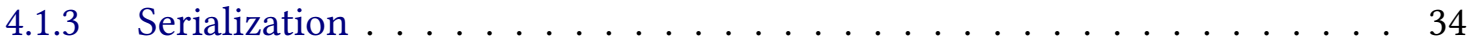

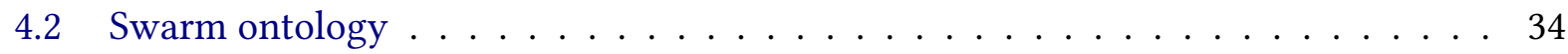

$4.2 .1 \quad$ Structure . . . . . . . . . . . . . . . . . . . 34

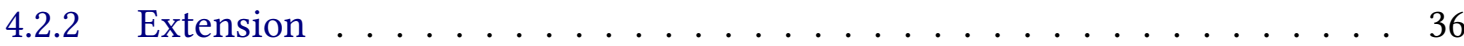

4.3 Query description model . . . . . . . . . . . . . . . . . 36

$4.3 .1 \quad$ Structure . . . . . . . . . . . . . . . . . 37

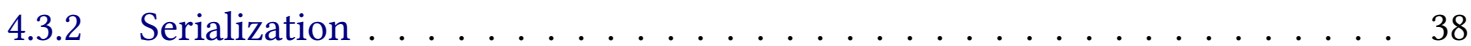

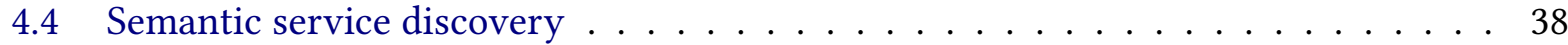

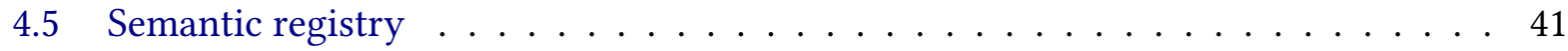

$4.5 .1 \quad \mathrm{RDF}$ generator $\ldots \ldots \ldots \ldots \ldots \ldots \ldots$

4.5 .2 Query generator . . . . . . . . . . . . . . . 42

4.5 .3 Semantic inference . . . . . . . . . . . . . . . . 42

4.6 Score computation . . . . . . . . . . . . . . . . . . . . . 42

4.6 .1 Type equivalence score . . . . . . . . . . . . . . . . . . 43

4.6 .2 Geolocation score . . . . . . . . . . . . . . . . . . 45

4.6 .3 Price . . . . . . . . . . . . . . . . . 46

4.6 .4 Reputation score . . . . . . . . . . . . . . . . . . 46

4.6 .5 Combined score . . . . . . . . . . . . . . . . . . . 47

4.7 Code generation module . . . . . . . . . . . . . . . . . . 47 


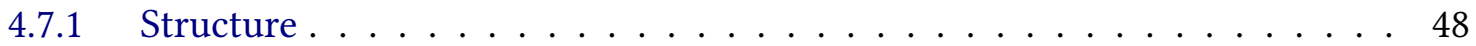

4.7 .2 Outcome ......................... 48

4.8 Service invocation . . . . . . . . . . . . . . . . . . . . . . . 49

4.9 Mediation mechanism . . . . . . . . . . . . . . . . . . . 50

4.10 Service composition . . . . . . . . . . . . . . . . . . . . 51

$5 \quad$ Implementation and use case $\quad 53$

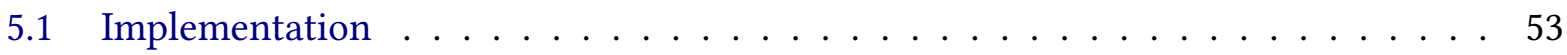

5.1 .1 Code generator . . . . . . . . . . . . . . . . 53

5.1 .2 Semantic registry . . . . . . . . . . . . . . . . . 54

5.1 .3 Swarm ontology . . . . . . . . . . . . . . . . 55

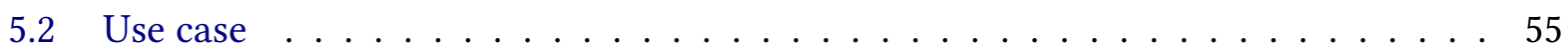

$5.2 .1 \quad$ Use case description: Lost cat . . . . . . . . . . . . . . . . 55

5.2 .2 Participants . . . . . . . . . . . . . . . . 55

5.2 .3 Using the framework . . . . . . . . . . . . . 56

6 Results $\quad 61$

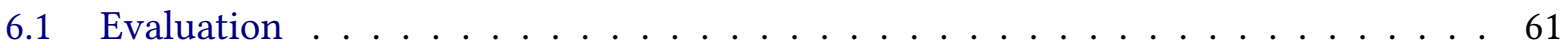

6.1 .1 Programming effort . . . . . . . . . . . . . . . . 61

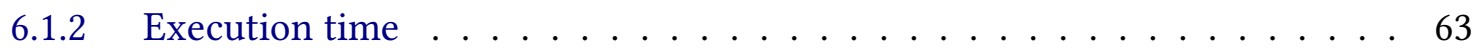

6.1 .3 Storage ........................... 64

6.2 Comparison with other works $\ldots \ldots \ldots \ldots \ldots$

7 Conclusions $\quad 69$

7.1 Synthesis . . . . . . . . . . . . . . . . . . . 69

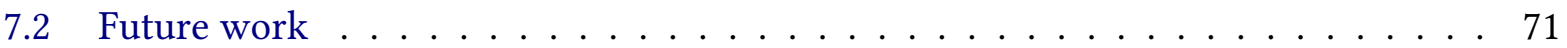

$\begin{array}{ll}\text { Bibliography } & \mathbf{8 1}\end{array}$ 


\section{List of Abbreviations}

$\begin{array}{ll}\text { API } & \text { Application Programming Interface } \\ \text { IoT } & \text { Internet of Things } \\ \text { CoAP } & \text { Constrained Application Protocol } \\ \text { GUI } & \text { Graphical User Interface } \\ \text { HTTP } & \text { Hypertext Transfer Protocol } \\ \text { IDE } & \text { Integrated Development Environment } \\ \text { RDF } & \text { Resource Definition Framework } \\ \text { REST } & \text { Representational State Transfer } \\ \text { SOAP } & \text { Simple Object Access Protocol } \\ \text { SPARQL } & \text { SPARQL Protocol and RDF Query Language } \\ \text { WoT } & \text { Web of Things } \\ \text { XML } & \text { eXtensible Markup Language }\end{array}$




\section{List of Figures}

1.1 Generic Internet of Things architecture . . . . . . . . . . . . . . . . . 1

1.2 Simple communication between human and IoT network. . . . . . . . . . . . . 2

1.3 Overview of the main challenges in the Swarm project. The main challenges of this thesis are highlighted in dark grey. . . . . . . . . . . . . . 4

1.4 Our framework helps programmers to create rich interaction in IoT applications. 4

2.1 Decentralized structure of the Swarm. . . . . . . . . . . . . . . . . 8

2.2 Structure of the Swarm Broker. . . . . . . . . . . . . . . . . . . . . . . . . 11

2.3 Architecture of the CoAP-HTTP proxy for the Swarm. a) High-level diagram of the CoAP-HTTP communication. b) Data workflow in both directions. . . . . . . 12

2.4 High-level diagram of the Swarm Minimum Broker. . . . . . . . . . . . . . . . 13

2.5 Architecture of the access control module of the Swarm. . . . . . . . . . . . . . 14

3.1 Different types of device interaction. . . . . . . . . . . . . . . . 22

3.2 Main components of the proposed framework . . . . . . . . . . . . . . . 24

3.3 Main use cases of the framework from the developer point of view. . . . . . . . . 27

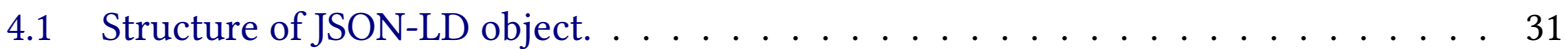

4.2 Example of a semantic service description. . . . . . . . . . . . 35

4.3 Swarm ontology. . . . . . . . . . . . . . . . . . . . . 37

4.4 Example of a semantic query description. . . . . . . . . . . . . . . . . . 39

4.5 Sequence diagram of the semantic service discovery . . . . . . . . . . . . . 41

4.6 Architecture of the Semantic Registry service. . . . . . . . . . . . . . . . . . . 42

4.7 Architecture of the Swarm code generator. . . . . . . . . . . . . . . . . . 48

4.8 Functioning of Service invocation module . . . . . . . . . . . . . . . . . 50

4.9 An example of the mediation mechanism. . . . . . . . . . . . . . . 51

4.10 Declarative composition of services. . . . . . . . . . . . . . . . . 52

5.1 Excerpt from the generated service code, showing the operation implementation. 54

5.2 Services that participate in the use case . . . . . . . . . . . . . . . . 56

5.3 Service description of the VisionFinder service. . . . . . . . . . . . . . . . 57

5.4 Declarative composition of services in the Swarm . . . . . . . . . . . . . . 58

5.5 Excerpt from the ontology used in the Lost cat use case. . . . . . . . . . . . . . . . 59 
6.1 Comparison of lines of code for the same application before and after using the framework. . . . . . . . . . . . . . . . . . . . 63

6.2 Comparison of execution time for tasks with and without the use of the framework. 64 


\section{List of Tables}

4.1 Non-functional properties categorized by O'Sullivan et al. (2005) compared to our service description model. . . . . . . . . . . . . . . . 30

4.2 Properties describing the functionality of a service. . . . . . . . . . . . . . 39

4.3 Parameters used to compute the score for candidate services. . . . . . . . . . . 43

4.4 Matching degrees between concepts. . . . . . . . . . . . . . . . . . . 44

4.5 Partial score matrix for type matching. . . . . . . . . . . . . . . . 44

6.1 Comparison of lines of code between the different tasks performed in the frame-

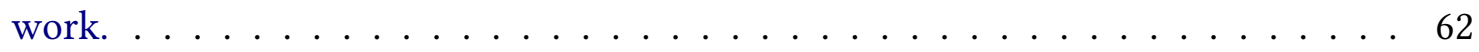

6.2 Comparison of the features of our framework with similar works. . . . . . . . 67 
Chapter 1

\section{Introduction}

Everyday objects are today provided with microprocessors and Internet connection, unveiling the potential of a global network of devices which interact in new ways; this paradigm is called the Internet of Things (IoT). In the IoT, a wide range of devices, mainly resource-constrained, connected to others to cooperate and reach common goals (Giusto et al., 2010). Current applications of the IoT include transportation, logistics, healthcare, smart environments (home, office, manufacturing), and social applications.

As several reviews show (Aggarwal et al., 2013; Atzori et al., 2010; Sethi and Sarangi, 2017), most IoT architectures are mainly cloud-centric. In general, sensor readings are transmitted to the cloud, which stores and analyses the data. After a decision-making stage, actions are taken, by sending commands to the actuators of the network. Figure 1.1 illustrates this generic architecture. Similarly, Taivalsaari and Mikkonen (2017) highlight the evolution of the cloud-centric data-centric Internet of Things towards the so called Programmable World, where smart edge devices become programmable components of complex cyberphysical systems. They also identify several challenges for software developers in the future Internet of Things.

Figure 1.1: Generic Internet of Things architecture.

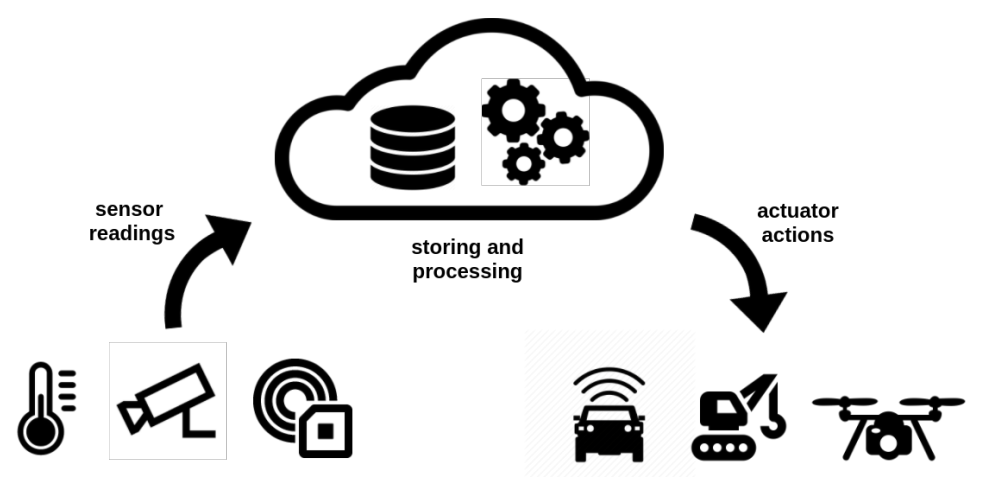

On the other hand, the latest IDC Worldwide Internet of Things Spending Guide forecasts a global spending of USD 1 trillion in 2022 (Siviero et al., 2019). The report also points hardware, software, 
data analysis and services as the main target of investments by companies. The potential number of people involved in the development of the IoT is also increasing. According to the United Nations (UN HABITAT, 2016), urban population will grow 12\% in the next 30 years.

In this context, Tsiatsis et al. (2019) recognize the importance of overcoming the barriers for the creation of IoT solutions in the real world. These barriers are related to the large number of heterogeneous devices and the diverse forms of device organization in an IoT system. Besides the training of skills for IoT development, it is necessary to create new frameworks to overcome the challenges of the future IoT. The present thesis aims to contribute in that direction.

\section{The Swarm project}

In order to provide a software infrastructure for the scenario described above, the Swarm platform (Costa et al., 2015b; Lee et al., 2014) was created aiming to be an organic, heterogeneous and decentralized network of things. The Swarm is an ongoing project with an extensive list of challenges, described in Section 1.1. The ultimate objective of the Swarm is to create an intelligent network of resource-sharing devices for the future Internet of Things. Moreover, to contribute to eliminate the barriers of access to a large network of devices, one of the main commitments of the Swarm is to provide rich and high-level communication with people, while encapsulating the complexity of device communication and organization to accomplish sophisticated tasks. This envisioned communication between human and network is illustrated in Figure 1.2. Our framework is designed with the objective of providing concrete advances towards the realization of this communication. Nonetheless, given the complexity of such effort, at this point we focus on providing the necessary tools for a programmer to create this device interaction in an effective way, as depicted in Figure 1.4.
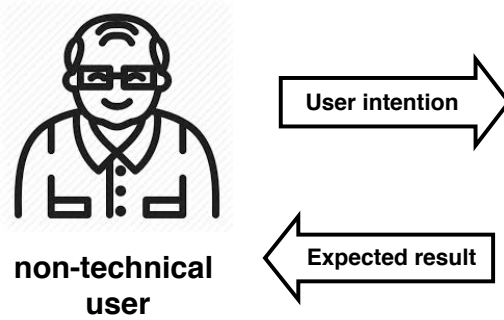

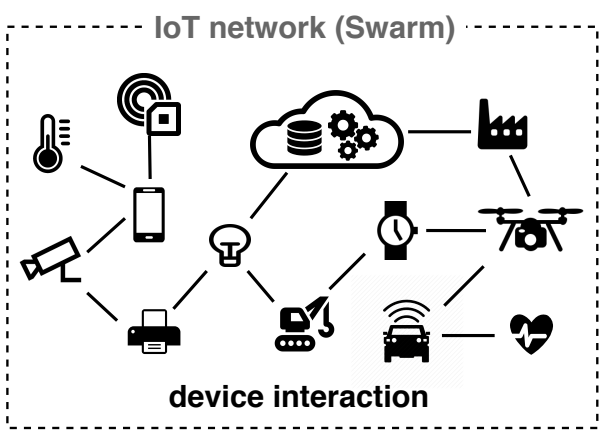

Figure 1.2: Simple communication between human and IoT network. 


\subsection{Challenges}

The framework proposed in this thesis aims to solve the challenges related to device interaction in the Swarm. Our understanding of device interaction refers to different forms of communication between devices with the purpose of performing a more complex task. We identify four types of interaction, summarized as follows:

- Registration is the process of a new device to enter into an existing IoT network, using a service description model.

- Discovery is the search of a specific device in the network that fulfills the desired characteristics. It involves a service and query description mechanism.

- Composition is the combined use of more than one device to perform a task.

- Mediation is the communication of otherwise incompatible devices with the help of an adaptation layer.

These interaction types are fully described in Section 3.1. The long-term objective of this framework is to provide the necessary models, specifications, libraries and tools to create an intelligent network of cooperating devices. Figure 1.3 shows the challenges of the Swarm project (ellipses), highlighting the challenges of this thesis in (dark grey ellipses), which are summarized as follows:

- Interoperability - The proliferation of heterogeneous and incompatible applications is a common problem in current Internet of Things. To contribute with the solution of this problem we propose the use of a semantic-based unified model for service API, which can be adopted with minimum effort, while not requiring previous knowledge on ontologies from the developer.

- Composability - After having interoperable devices, the next challenge is to combine the functionality of several devices to accomplish a larger task. Our framework aims to create models and tools for service composition, including registering, discovery, and mediation.

- Rapid application development - As Giang et al. (2015) identifies, there is a lack of tools for agile development of IoT applications, that include the distributed and heteroge- 


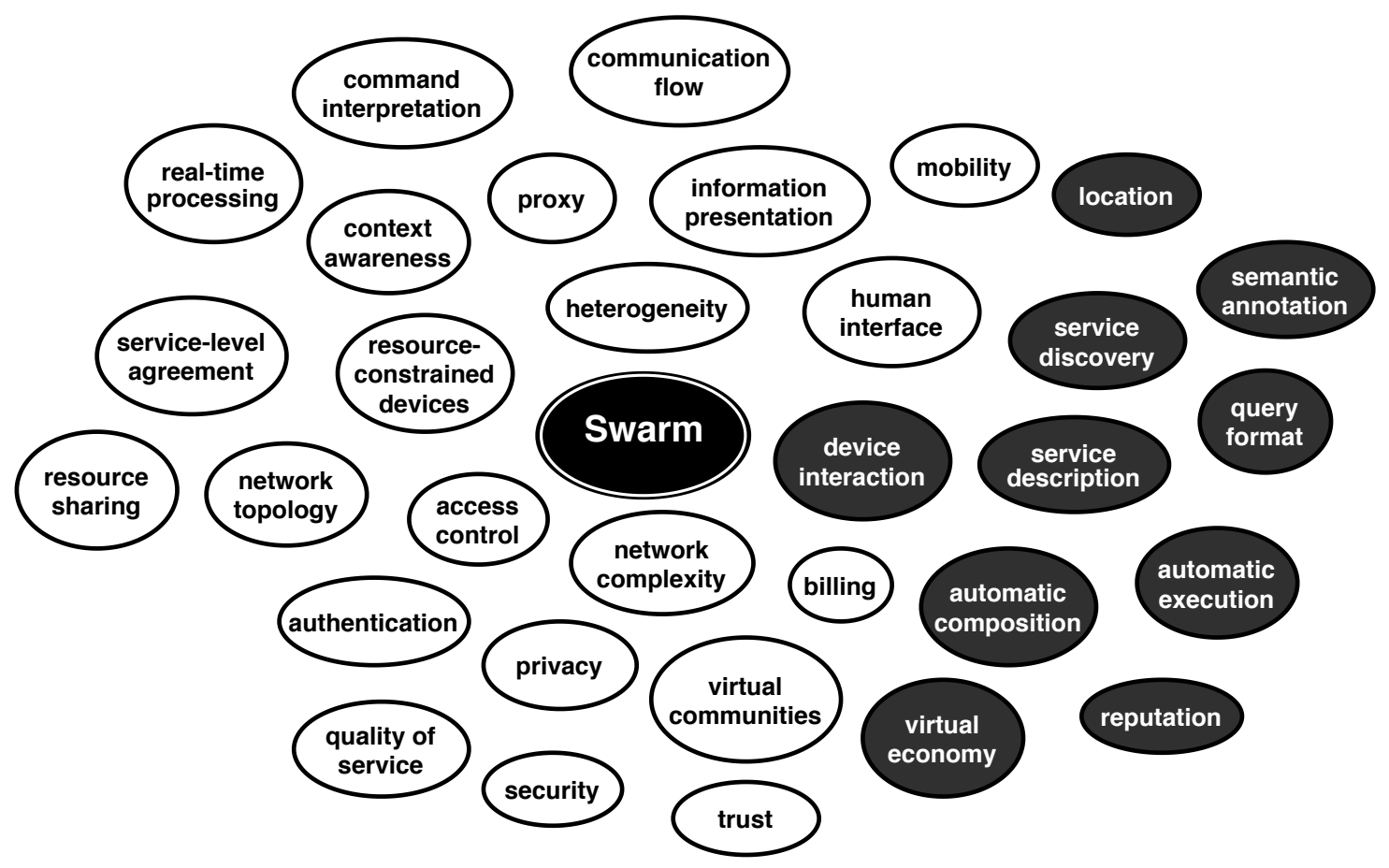

Figure 1.3: Overview of the main challenges in the Swarm project. The main challenges of this thesis are highlighted in dark grey.

neous nature of devices. Creating a general purpose set of tools for IoT applications is a challenging task, and in this work we focus on produce a framework adapted to the Swarm model. These ideas can be adapted and generalized to other types of IoT platform.

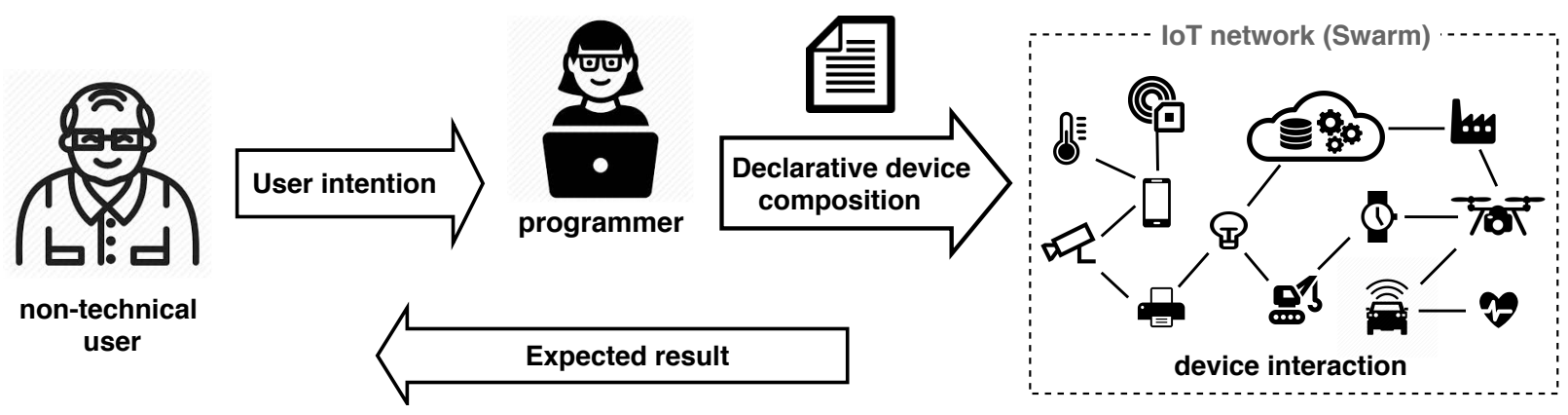

Figure 1.4: Our framework helps programmers to create rich interaction in IoT applications.

\subsection{Problem definition}

The problem that this thesis intends to solve can be stated as follows:

how to create a framework that permits the agile development of IoT applications with flexible interaction among devices.

In this problem definition, we consider the following concepts

- Agile development - Referring to the use of simple, legible, easy-to-understand models 
for the description of the application. It also includes the creation of tools that automate repetitive, redundant and verbose tasks to save programming effort.

- Flexible - Related to the possibility of getting equivalent results for a query, thus extending the coverage of the target results.

- Interaction - Related to the diverse forms of communication among devices, which include discovery, composition, and mediation. It also includes the social aspect of interaction, such as the motivation and incentives of devices to choose the conditions for cooperation with other devices.

\subsection{Objective}

The main objective of this work can be stated as:

1. Provide the Swarm with platform service to find other services based on a taxonomy of functionalities.

2. Provide a mechanism for automatic invocation of services in the Swarm, based on a machinereadable API.

3. Create a mechanism for services in the Swarm to autonomously organize and combine functionalities in order to perform high level tasks.

\subsection{Hypothesis}

Based on recent successful uses of semantics in diverse aspects of the Internet of Things, such as interoperability (Noura et al., 2019), discovery (Zorgati et al., 2019), data exchange (Kolbe et al., 2019) we propose the following hypothesis:

using semantics as a driving technique, we can create a communication framework that permits flexible discovery, automatic execution, and composition of services in an IoT network.

\subsection{Contributions}

The contributions of this work are summarized below:

- The definition and implementation of a framework for discovery, execution, and compo- 
sition of services in an Internet of Things network. This is the main contribution of this work and comprehends the other contributions.

- A simple description format for declarative description of semantic services.

- A basic ontology for semantic service description.

- The concept of service mediators, that permit communication among otherwise incompatible services.

- A declarative mechanism for service composition.

- A tool for code generation from declarative service description.

- A ranking mechanism for choosing the most suitable device in an interaction process.

- An ontology for geolocation of devices in an IoT network.

\subsection{Thesis structure}

This thesis is structured as follows. Chapter 2 introduces the Swarm project and explores relevant work related to this thesis. In Chapter 3 we provide an overview of the main concepts and the functioning of the proposed framework. Chapter 4 explains in detail the components of the framework. Next, in Chapter 5 we describe the implementation of the framework and its integration into the Swarm platform. The results of this work are presented in Chapter 6, and Chapter 7 provides a final discussion on the thesis. 


\section{Basic concepts and technologies}

\subsection{The Swarm}

The swarm term was first proposed by Rabaey (2011) to refer to sensory found at the edge of the cloud, and identified the opportunity for the realization of areas such as cyber-physical systems, cyber-biological systems, immersive computing and augmented reality. Rabaey also identified the key challenges to leverage the potential of this swarm. Those challenges include the transparent integration of sensors with the environment; the self-contained and self-sustaining nature of the nodes; the non-linear, non-deterministic adaptive and complex nature of the swarm network; and the creation of a general framework that enables a seamless communication among heterogeneous devices. Subsequent work led to a more concrete definition of the Swarm. Lee et al. (2014) proposed an initial architecture for the Swarm in the context of a larger project called TerraSwarm. They also outlined a common framework for resource sharing among devices, called SwarmOS. The architecture of the SwarmOS framework was further developed by Costa et al. (2015b), complementing the already existing distributed storage system (data plane) with a module responsible for the sharing and management of resources (control plane).

\subsubsection{The Swarm project}

The Swarm project ${ }^{1}$ is the umbrella project where this thesis has its basis. This project is under active development in the Centre of Interdisciplinary and Interactive Technologies of the University of São Paulo (CITI-USP) ${ }^{2}$ in cooperation with the SwarmLab at University of Berke$l^{3} y^{3}$. The main objective of the Swarm project is to overcome the limitations of cloud-centric IoT architectures.

The Swarm is defined as a self-adaptive network for autonomous smart objects, where devices do

\footnotetext{
${ }^{1}$ http://iotswarm.info/

${ }^{2}$ http://www.lsi.usp.br/citi/en/

${ }^{3}$ http://swarmlab.berkeley.edu/
} 
not rely on the cloud for storage and processing, but instead, part of this work can be performed in the device itself. The Swarm is a heterogeneous network constituted of different kinds of devices, with variable computing power and energy capabilities. In Figure 2.1 we illustrate the general structure of the Swarm. Making a parallel with swarm of bees, with specialized bees contributing to a common goal, the Swarm is composed of specialized devices whose interaction solves a common problem. The Swarm network behaves like an organism and shows an organized behavior that results in an emergent collective intelligence.

Figure 2.1: Decentralized structure of the Swarm.

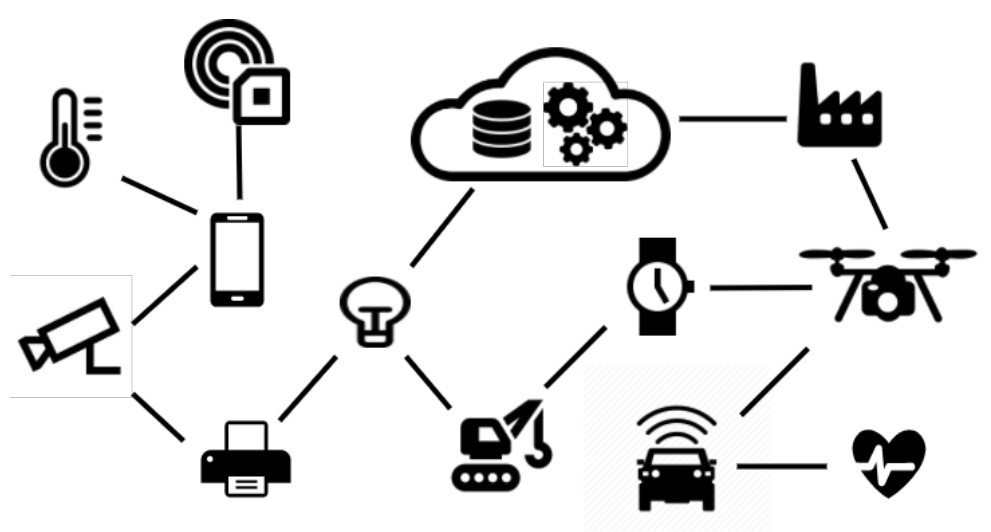

Device functionalities are exposed and shared to the network as services. Thus, the Swarm can be seen as a large network of interacting services. Every service is specialized in a specific functionality, and many services can perform similar or equal functionalities. The true potential of the Swarms resides in the composition of services, which dramatically extends the functionalities offered by the network. Given the intersections with the microservices architectural style, we adopted many of its concepts for the Swarm, such as the use of services as the main building block; loose coupling and high cohesion; decentralized governance; decentralized data management; and evolutionary design (Fowler and Lewis, 2014).

Interaction among devices is performed opportunistically, with no prior agreement. The connection among devices is established in real-time, based on the availability of devices in the network. As a response to an external event, devices in the network form groups in order to perform an action or give an answer. Although those groups formed are transient, the success of each interaction is recorded in the network and serves to build a measure of reputation for each device. 


\section{Key challenges}

Key challenges to achieve the Swarm vision, include a large variety of aspects that are currently under development, which are summarized as follows:

- Heterogeneity - Heterogeneity in the Swarm is present in many aspects. Differences in participant devices can be found in functionalities, computing power, description formats, communication protocols, and response time, among others. The project aims to provide a common communication framework that takes advantage of existing open and mature standards. A particular focus on resource-constrained devices is given, since the installation of software on those devices is not straightforward, and must be done with minimum impact.

- Security - Although the security challenge in computer systems is not new, in the context of the IoT and the Swarm it is even more challenging. Many devices do not have enough computing power and energy to support strong cryptographic algorithms, used in authentication and authorization. Additionally, the need for rapid development and release of products in the consumer market precludes the exhaustive testing of basic security capabilities in new products. Finally, as the Swarm network is composed of autonomous devices, policies for a fair interaction must be provided to avoid abuse.

- Device interaction - Once the barriers of heterogeneity and security have been overcome, the next challenge consists in guaranteeing the device interaction. This interaction happens in the service level, as services are the building block of the Swarm network. To effectively create an organic and self-organizing network of services, they must be capable of composing automatically, based on network demand. The first step to initiate an interaction is to perform a search for services (discovery). Then, services must be capable of invoking others automatically, based on its machine-readable description. Finally, services must be capable of automatically combining its capabilities to perform a higher level task.

- Resource sharing - After the device interaction is guaranteed in the Swarm network, the next step is the creation of a rewarding mechanism for service invocation. This effectively constitutes a network of cooperation among devices. New concepts arise in the 
network such as trust, virtual currency, billing, reputation, and a full virtual economic system. This phenomenon represents a perfect parallel to a growing trend in current world economy called sharing economy, which favors the sharing of physical resources over the acquisition of new ones. Resource sharing in the Swarm represents its digital equivalent. A consequence of resource sharing is a reduction of uncontrolled consumption and a better global use of resources.

- Human interface - The Swarm described so far is composed mainly of machine-tomachine (M2M) interactions. Nonetheless, the human component is essential as it is the main source of external events and request to the network. Innovative ways of interaction with this large network constitute a major challenge. Humans should have no barriers to take full advantage of the network, by asking actions in a high-level language. The format of the response and the establishment of a communication flow must also be carefully studied. The Swarm network must take advantage of the diversity of devices for acquisition and presentation of information.

\subsubsection{Swarm Broker}

One of the most important components in the Swarm platform is the Swarm Broker, which is a software installed in every IoT device and acts as a communication facilitator. Some functions provided by the Swarm Broker include service registry, semantic service discovery, access control policy enforcement, service contracting, and blockchain-based reputation. Additionally, the Swarm Broker provides a transaction model that mediates contracts between service consumers and providers which are through the service discovery mechanism. That interaction creates an economic model for resource sharing in the IoT. We define two categories of services in the Swarm: platform service, that constitute the core functionalities of the Swarm network; and application services, which are all other services that participate in the Swarm. Thus, the Swarm Broker can be seen as the collection of platform services. Figure 2.2 illustrates the landscape of platform services that constitute the Broker. As the Swarm network is composed of heterogeneous devices, the number of platform services varies across devices, according to its capabilities. Less powerful devices will provide fewer platform services. Current implementations include C, Lua, Java, and Elixir programming languages, with further targets are expected. 


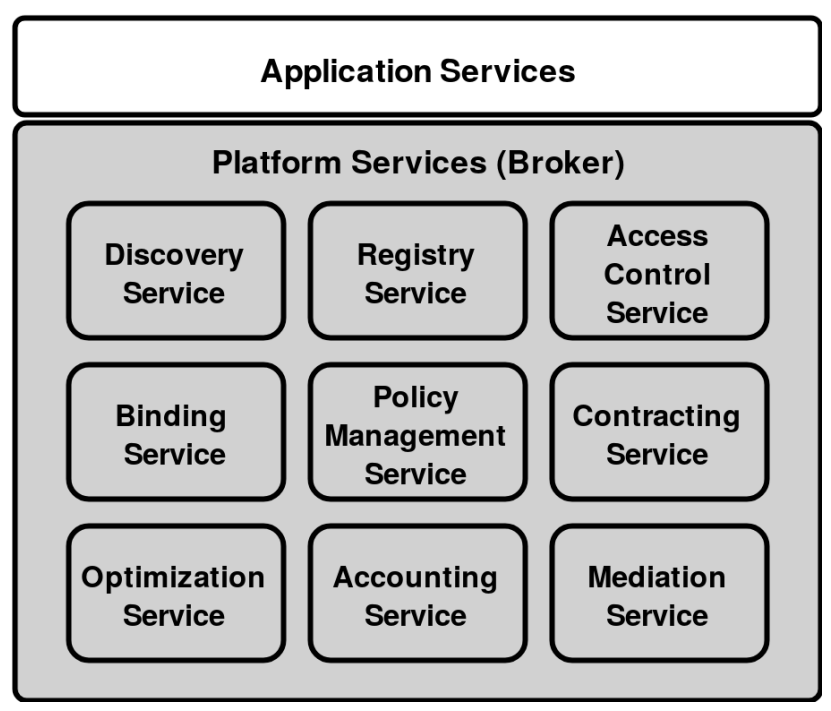

Figure 2.2: Structure of the Swarm Broker.

\subsubsection{Swarm economic model}

The rich interaction among the participants of the Swarm network poses important challenges to guarantee the soundness and integrity of device interactions. As a result, De Biase et al. (2018b) proposed an economic model for the Swarm, that aims to regulate the transactions of services in the network. Relevant concepts are part of this model, such as trust, reputation, rewarding mechanism, and billing. The introduction of a rewarding mechanism in the Swarm platform is of major importance for the service ecosystem, as it leverages a concrete incentive for resource sharing. Devices can share its functionalities either in exchange for the use of other devices, or to accumulate credits in the SwarmCoin currency. In the economic model of the Swarm, the Swarm Broker is responsible for linking the parties and for facilitating transactions. The price of a resource represents the number of credits necessary for the service provider to grant access to the service consumer. Credits belong to the owner and are used by the service to contract or purchase any service on behalf of its owner. The Swarm economic model is based on the price of a service and the reputation of both service consumer and provider, hence it is called the pricereputation model. A service provider defines the number of credits necessary to allow a third party to use it.

During the transaction process, reputation points evaluate the success of the operation. The price-reputation transaction is the simplest transaction defined for the Swarm framework: the consumer gets the service by paying a number of credits settled by the provider, depending on 
their behavior, they both get reputation points during the process. Regarding security, the distributed and decentralized nature of the Swarm requires a new model for transactions. Traditional technologies, such as the public key infrastructure (PKI), are not suitable as they demand a centralized certificate authority (CA). Accordingly, the Swarm adopted the blockchain technology, used in the Bitcoin cryptocurrency, to create a decentralized mechanism for trust in the economic model of the Swarm network (De Biase et al., 2018b). The advances in an economic model for the Internet of Things go in the same direction of a growing trend in the world economy: sharing economy. As in the physical world, the Swarm favors the digital sharing of resources over the acquisition of dedicated devices. As a consequence, it produces a reduction of device consumption and better global use of resources.

\subsubsection{CoAP-HTTP proxy.}

The Constrained Application Protocol (CoAP) is a standard application protocol, designed to implement RESTFul services in constrained devices, thus being an optimized alternative to HTTP. Esquiagola et al. (2017) proposed a proxy mechanism for the Swarm that permits seamless communication between services that implement both protocols. Figure 2.3-a illustrates the highlevel communication between services in the Swarm, grouped by the implemented protocol. Services send a request to the associated broker, which forwards it to the proxy for message translation and, in turn, forwards the message to the target service. That way, none of the services needs to know in advance that the communication is being performed through another protocol. Additionally, Figure 2.3-b shows the bidirectional implementation of the proxy, which permits CoAP-HTTP and HTTP-CoAP communication, thus extending the coverage of the Swarm network for resource-constrained devices.

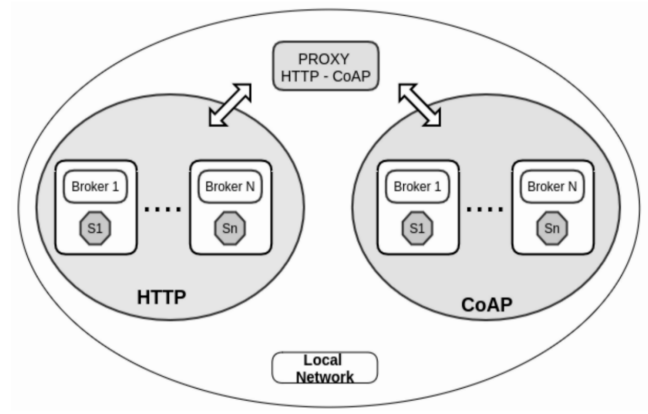

a)

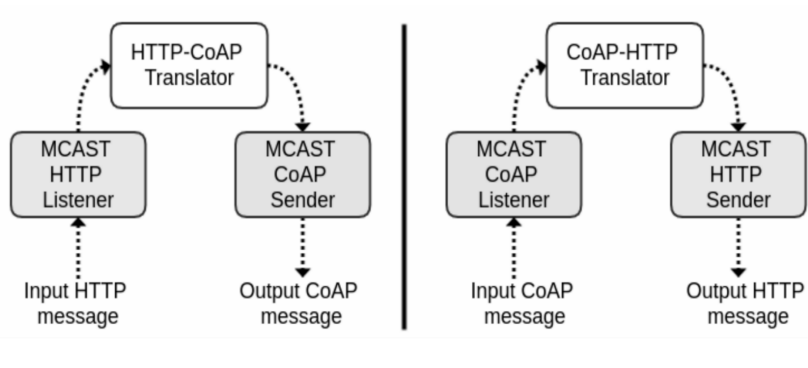

b)

Figure 2.3: Architecture of the CoAP-HTTP proxy for the Swarm. a) High-level diagram of the CoAP-HTTP communication. b) Data workflow in both directions. 


\subsubsection{Swarm minimum broker.}

To overcome the challenge of heterogeneity in the IoT, a Swarm Minimum Broker (MB) contains the core features necessary for a device to be part of the Swarm network. That way, devices with resource-constrained resources can use an external software proxy to translate communication (De Biase et al., 2018a). The basic requirements for Swarm participants are being discoverable and usable. Thus, a simplified implementation can provide support for requests through different protocols, such as HTTP and SSDP. Figure 2.4 illustrates the interaction between Minimum SwarmBroker and common Swarm Broker, focusing on the discovery process.

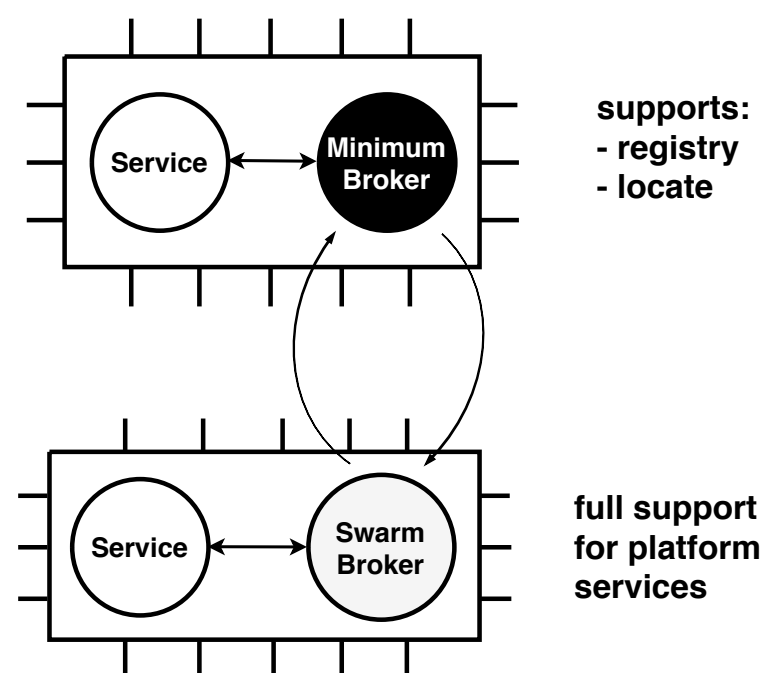

Figure 2.4: High-level diagram of the Swarm Minimum Broker.

\subsubsection{Access control model}

Security in the Swarm platform includes both the protection of exchanged messages and a flexible access control mechanism over shared resources. Furthermore, managing the access control of a large number of devices becomes a significant challenge. The access control mechanism of the Swarm platform is based on the Attribute-Based Access Control (ABAC) model (Hu et al., 2015). In $A B A C$, a subject requests to perform operations on objects and is granted or denied, based on attributes of the participants. The referred attributes can be related to the subject, the object, environment conditions, and policies. The ABAC-based model created in the Swarm platform is called ABAC-them (Fedrecheski et al., 2018) and focuses on combining simplicity and expressiveness. The main characteristics of ABAC-them model are: 
- Enumerated policies - attribute enumeration allows the creation of policies that are easy to parse and to embed into small devices.

- Hierarchical attributes - allow the creation of high-level policies that are easier to write and understand. During execution time, low-level attributes present in access requests benefit from attribute hierarchies, which allow them to match with the high-level policies.

- Typed attributes - provides a counterbalance to policies that can grow large when using enumeration, such as those involving numerical ranges.

- Multiple attributes - very specifically, this feature allows easy creation of conjunctions when using enumerated policies.

The ABAC-them model was implemented within an architecture based on the NIST recommendation for ABAC systems (Hu et al., 2014). The architecture contemplates four main components. The Policy Decision Point (PDP) evaluates policies that are managed through the Policy Administration Point (PAP), while the Policy Information Point (PIP) is responsible for gathering context and other attributes, and the Policy Enforcement Point (PEP) intercepts requests and verify their permission with the PDP. While the original NIST architecture proposes that PDP, PIP, and PAP reside in an authorization server, the SwarmOS implementation puts all points inside the IoT device, thus enhancing its autonomy and security. Figure 2.5 illustrates the current architecture of the ABAC-them model in the SwarmOS.

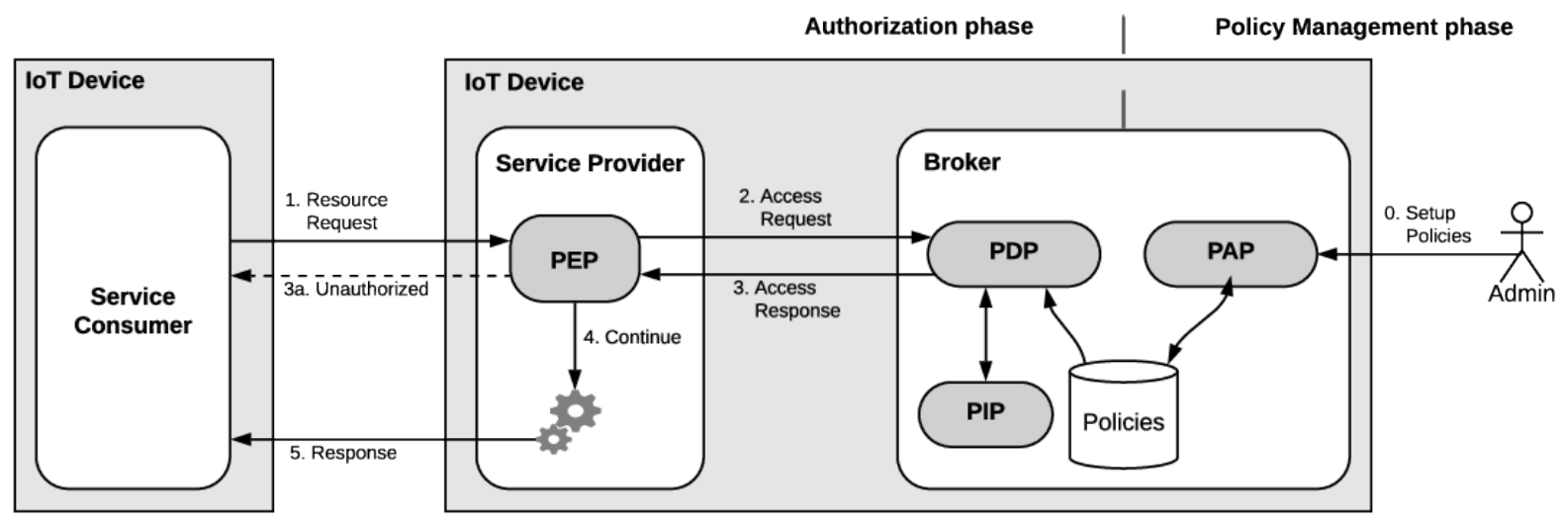

Figure 2.5: Architecture of the access control module of the Swarm. 


\subsection{Related visions}

Several ideas and initiatives intersecting with the Swarm network were previously proposed. In this section we review work with focus on decentralized, self-adaptive and organic systems, which we consider the features that better characterize the differentiation of the Swarm from centralized IoT systems.

Weiser (1995) proposed the term Ubiquitous Computing as a future reality with computers strongly present in human activities while being almost invisible. Such invisibility refers to not having prominence in the interaction, but acting as a facilitator. Weiser envisioned computers as invisible and ever-present tools for human tasks. Despite the experimental work done to demonstrate this concept, the technological enablers were not enough at that time.

Horn (2001) while working at IBM, identified the important challenge that complexity represented for the future of computing systems. It manifests in overlapping connections, interactions, and dependencies of heterogeneous systems, whose managements goes beyond human capabilities. According to him, the solution to manage complexity is to create systems that mimic the functioning of the human autonomic nervous system. An essential feature of an autonomic system is its self-management. It is analogous to the regulation of heart rate or body temperature in response to external conditions, to maintain a steady internal state. A system of this nature should implement four basic features: self-configuration, self-optimization, self-healing, and selfprotection. Elements of an autonomic system are also autonomic and interact according to its own goals. The need for self-managed systems gains a renewed importance in the context of increasing complexity of the Internet of Things. The Swarm vision shares many concepts with Autonomic Computing.

Boley and Chang (2007) proposed an analogy between biological and digital ecosystems. Several different species co-exist in an environment. Species get necessary resources from environment and also preserve it. Members of a species are individuals. Four aspects are essential for ecosystems: interaction and engagement, that includes sharing resources and forming groups, thus guaranteeing the social well-being; balance, that maintains the harmony of the ecosystem and contributes to its sustainability; domain clustered and loosely coupled, individuals, on their own 
choice, form groups according to similar interests and objectives; and self-organization, which implies the autonomy of species, and the coordination through swarm intelligence. In digital ecosystems a swarm is defined as a set of agents interacting and engaged agents that solve a common problem. Also, Boley and Chang highlight the advantages of using semantic web technologies for information exchange, modeling of attributes and integrity check. The interaction among devices inspired in swarm intelligence and the self-organization capabilities also served as inspiration for the device interaction in the Swarm.

Atzori et al. (2012) work share some similarities with the Swarm vision. They study the relationships of cooperation among IoT devices. Unique identification and description of resources are necessary for this cooperation. However, the proposed architecture for Social IoT relies on a central server that is responsible for composing services. That server has three modules a database with device information; a module for device interaction; and an application layer that provides service composition. The Swarm vision shares the treatment of devices as participants of a social group and extends this idea to create the concepts of trust, reputation and even a virtual economy.

Zambonelli (2012) calls superorganism a system formed by a group of organisms that exhibit intelligent and adaptive collective behavior, giving as examples, colonies of ants, honeybees, and termites. Zambonelli foresaw the emergence of urban superorganisms, composed by sensing devices deployed across the city, enriched by information from human users, in order to create an aggregate system capable of providing value added to its environment. The author also highlights the challenges to achieve this vision, such as models and software for sociotechnical processes, the understanding the human-device interaction in large scale, and, incentives for sharing, including a monetary approach. The Swarm vision shares some challenges with this approach, and many of the ideas served as inspiration.

In the last decade, the cloud has emerged as the main responsible for storing and analyzing information. The IoT adds a communication layer between the physical and logical world, by adding sensor and actuator devices to software systems. In a first stage of the IoT, resource-constrained devices act as providers of data that feeds the cloud. Currently, IoT devices are empowered and less dependent on third parties. Evidences of this new paradigm can be found in recent work, 
named as fog computing (Bonomi et al., 2012) and edge computing (Shi et al., 2016). In fog computing, part of the processing and storage is delegated to general purpose computers and network equipment closer to IoT devices. In edge computing, devices themselves run the services needed for the operation of the network. The definitions of edge and fog have points of intersection and agree on performing computing closer to the devices. The Swarm vision has many coincidences with the edge computing paradigm. Both aim to make the devices less dependent of the cloud, and favors a more decentralized IoT. The Swarm, however, goes beyond the ideas of independence and decentralization. We stress the cooperation of devices and the emergence of collective intelligence.

\subsection{Other related frameworks}

In this section we review other related frameworks, in order to explore the similarities with the present work. In Chapter 6 we provide a comparison and in Table 6.2 we present a summary of this analysis.

\subsubsection{OWL-S}

OWL-S was one of the first initiatives to integrate the Semantic Web into existing Web service technologies (Martin et al., 2004). As a result, OWL-S proposes an ontology to describe a service using the Web Ontology Language $(\mathrm{OWL})^{4}$. Due to the time of creation of OWL-S, it was designed to interact with SOAP Web services, which are considered heavyweight and are not suitable for IoT applications. Several efforts based on the OWL-S project proposed important contributions, such as automatic service composition (Klusch et al., 2005; Sirin et al., 2004) and service mediation (Vaculín and Sycara, 2007).

\subsubsection{WSMO}

The WSMO project was a European initiative (Jos de Bruijn et al., 2005) that aimed to provide a complete platform for the development of semantic Web services, including an ontology (WSMO), a powerful modeling language for the specification of service descriptions and queries (WSML), an Eclipse-based programming environment (WSMT), and a runtime execution environment (WSMX). Similarly to OWL-S, the WSMO project was targeted to SOAP projects, nonetheless,

\footnotetext{
${ }^{4}$ https://www.w3.org/OWL/
} 
further initiatives proposed integration with RESTful services, such as WSMO-lite (Vitvar et al., 2008) and hRESTS (Kopeckỳ et al., 2008).

\subsubsection{Hydra}

The Hydra ${ }^{5}$ project (Lanthaler, 2013) aims to provide a semantic description of Web APIs for RESTful services. This project was created by the same author of the JSON-LD model ${ }^{6}$, and was built on top of it. JSON-LD provides a general purpose format to describe linked data, in the form of Web resources. The Hydra project constitute a common vocabulary to describe Web APIs with semantic annotations, by modelling a service, its operations, input and output parameters, grounding information, and human-friendly textual descriptions. The service description that we propose in this thesis has been inspired by the Hydra Core Vocabulary.

\subsection{4 oneM2M}

The oneM2M ${ }^{7}$ initiative consists on an international partnership that aims to provide a global M2M service platform, by developing a set of technical specifications that constitute a service layer to be used in M2M applications. The range of specifications developed by the oneM2M project includes protocols, APIs, security and privacy, interoperability, data collection, and device management. Additionally, oneM2M provides a set of tools to aid in the creation of applications, including clients in different programming languages. Regarding the interoperability efforts, the oneM2M project provides a Base Ontolog $y^{8}$ created in order to be extended by external organizations when creating specific domain applications. Also, oneM2M provides semantic discovery of devices through the use of SPARQL queries.

\subsubsection{SWoTPAD}

The SWoTPAD project (Silva et al., 2019), proposes a semantic framework to aid the development of IoT applications. SWoTPAD is inspired by the Semantic Web of Things (SWoT) paradigm, that uses RESTful services to model the functionalities of IoT devices. Another inspiration for SWoT$\mathrm{PAD}$ is the WSMO project, that provides a rich language (WSML) for creating semantic Web services. Despite being powerful, the WSML language has several disadvantages, related to its

\footnotetext{
${ }^{5}$ https://www.hydra-cg.com/spec/latest/core/

${ }^{6} \mathrm{http}: / /$ www.w3.org/json-ld11

${ }^{7}$ https://www.onem2m.org/

${ }^{8}$ https://git.onem2m.org/MAS/BaseOntology
} 
complexity. In response to that, the SWoTPADL language offers a simplified, yet powerful notation for service description, including service hierarchies and instances. One of the main highlights of the SWoTPAD framework is the implementation of automatic composition of services through the use of a hierarchical task planning (HTN) algorithm, and the generation of modules for automatic discovery.

\subsubsection{OSF}

The OSF project (Mayer et al., 2017) was proposed to leverage the use of semantic technology into the Internet of Things. The main tasks that OSF framework provides include the acquisition, augmentation, maintaining, and reasoning over semantic knowledge. The OSF contains a set of core ontologies, common to several application domains, which are distributed in knowledge packs. Additionally, OSF provides a visualization tool, including 2D and 3D visualizations. That project was tested with an Industrial IoT application, integrating models from different subdomains of an application for workplace safety.

\subsubsection{ReLL}

In contrast to the focus on WS-* style Web services of initial projects, such as OWL-S and WSMO, the Resource Linking Language (ReLL) (Alarcon et al., 2011) proposed a mechanism for RESTful service composition. The ReLL annotation for services is based on XML and later transformed to RDF, to be stored and queried through SPARQL. Additionally, ReLL uses a model based on Petri Nets for static service composition. 
Chapter 3

\section{Framework definition}

In this chapter we present a high-level overview of the functioning of the framework, introducing the main concepts and how they relate to accomplish the objectives of this work. The framework we propose was designed to assist developers to build IoT applications with rich device interaction. Although this framework was initially conceived to be integrated into the Swarm platform, the ideas presented here can be applied independently without loss of generality.

We begin by stating our understanding of device interaction, then we present the basic building blocks of the framework, and discuss the use of the framework.

\subsection{Definitions}

The Internet of Things in the forthcoming years will be composed of smarter devices that form decentralized networks. Accordingly, the Swarm vision, presented in Section 2.1, stresses the importance of device interaction to accomplish the goal of producing a large self-organizing network of smart agents. Next we define the concepts used in our work.

\subsubsection{Device}

We consider as device any piece of hardware with enough computing power and networking capabilities to send and receive messages to other devices. This definition also corresponds to the commonly used for thing in the Internet of Things literature. Similarly, as we adopted a serviceoriented approach for the framework architecture, every device that participates in the network exposes its functionality through a service, particularly a RESTful web service (Fielding and Taylor, 2002). As such a service is a representative of the underlying device where it runs, therefore, in this work we use the terms device, thing and service interchangeably as we are focused on the interactions the devices have through the services that represent them. 


\subsubsection{Network}

We define the network as the set of devices that are mutually reachable through a common communication protocol. In the context of our work, we consider that these services also share an implementation of our software library and exchange messages following according to the object models specified by our framework.

\subsubsection{Framework}

We define the framework as the collection of all object model specifications, their communication flow, and the respective software implementation, which runs on the devices of the network.

\subsubsection{Interaction}

We define service interaction as the different types of communication among devices that occur in a network of things. The ultimate goal of device interaction is to combine the functionality of individual things in order to produce an articulated behaviour of the participants that eventually creates an intelligent network. Figure 3.1 shows the device interaction considered in the context of this thesis.

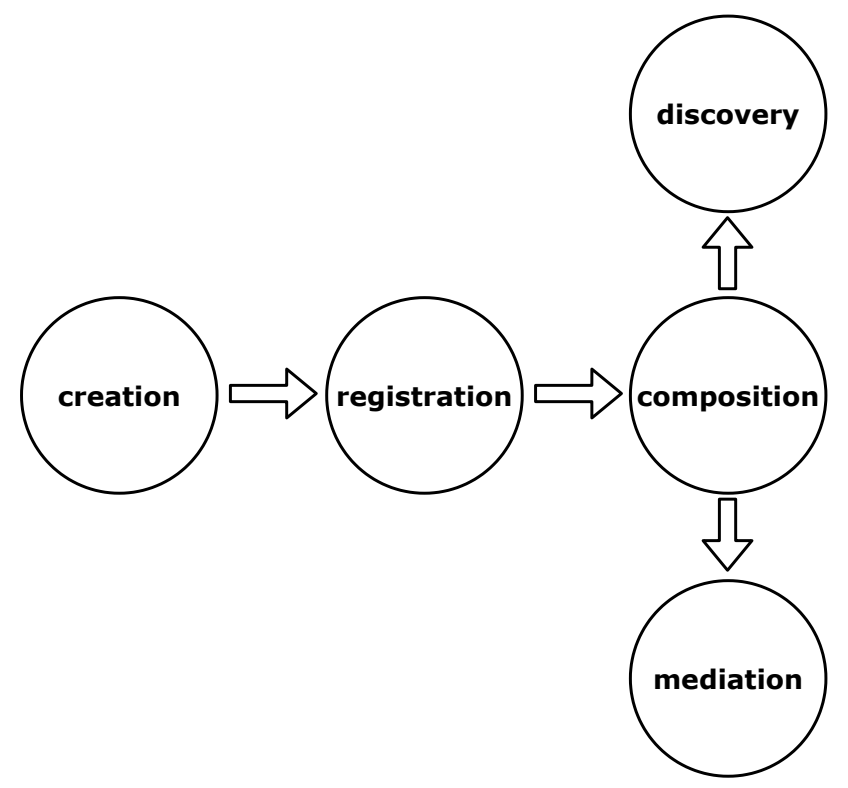

Figure 3.1: Different types of device interaction. 


\section{Creation}

Although it is not properly an interaction among devices, the creation of a service is the starting point in the lifetime of a service, and it represents the initial contact for the programmer with the framework. The service creation includes the definition of the service API, its implementation and execution in a concrete hardware.

\section{Registration}

Once a service is running, it needs to find other services in the network to interact. To accomplish this, the service scans the network to find other devices and sends its service description file to them. Among the network participants, there is one special service called semantic registry, that acts as a service directory, storing the description files of other participants.

\section{Discovery}

The discovery interaction consists in finding a service in the network that matches the desired functionalities based on a query file that specifies these parameters. The discovery interaction occurs with the participation of the semantic registry service, which contains the information of other participants in the network.

\section{Composition}

Service composition is one of the most powerful interactions and consists in combining the functionality of several services to act as a single software agent. Our framework helps the developer to define the composition of services in a declarative way. By defining the characteristics of the services to be composed, the framework performs a discovery process and then compose the execution of those services.

\section{Mediation}

A mediator service $M$ helps the composition of a pair of services $A$ and $B$ with different input and output (incompatible). To accomplish this, $M$ is specialized in transforming data. Formally, we express the input and output datatype of a services $A$ and $B$ as: $A: A_{\text {domain }} \rightarrow A_{\text {range }}$ and $B: B_{\text {domain }} \rightarrow B_{\text {range }}$. Then, the mediator service $M$ is defined as $M: A_{\text {range }} \rightarrow B_{\text {domain }}$. 
and applied to a service-oriented architecture. Mediator services are treated as special services in our framework, as there is no need to specifically declare them in a composition list. The use of service mediators greatly extends the composition potential of the network. It is important to note that the benefits of the mediation mechanism will depend on the active participation of the developers of the Swarm community to create and share these services.

\subsection{Main components}

Based on the interaction types defined in previous section, we identify the key components that serve as building blocks for the framework. A high-level overview of these concepts is depicted in Figure 3.2 showing the main relationships.

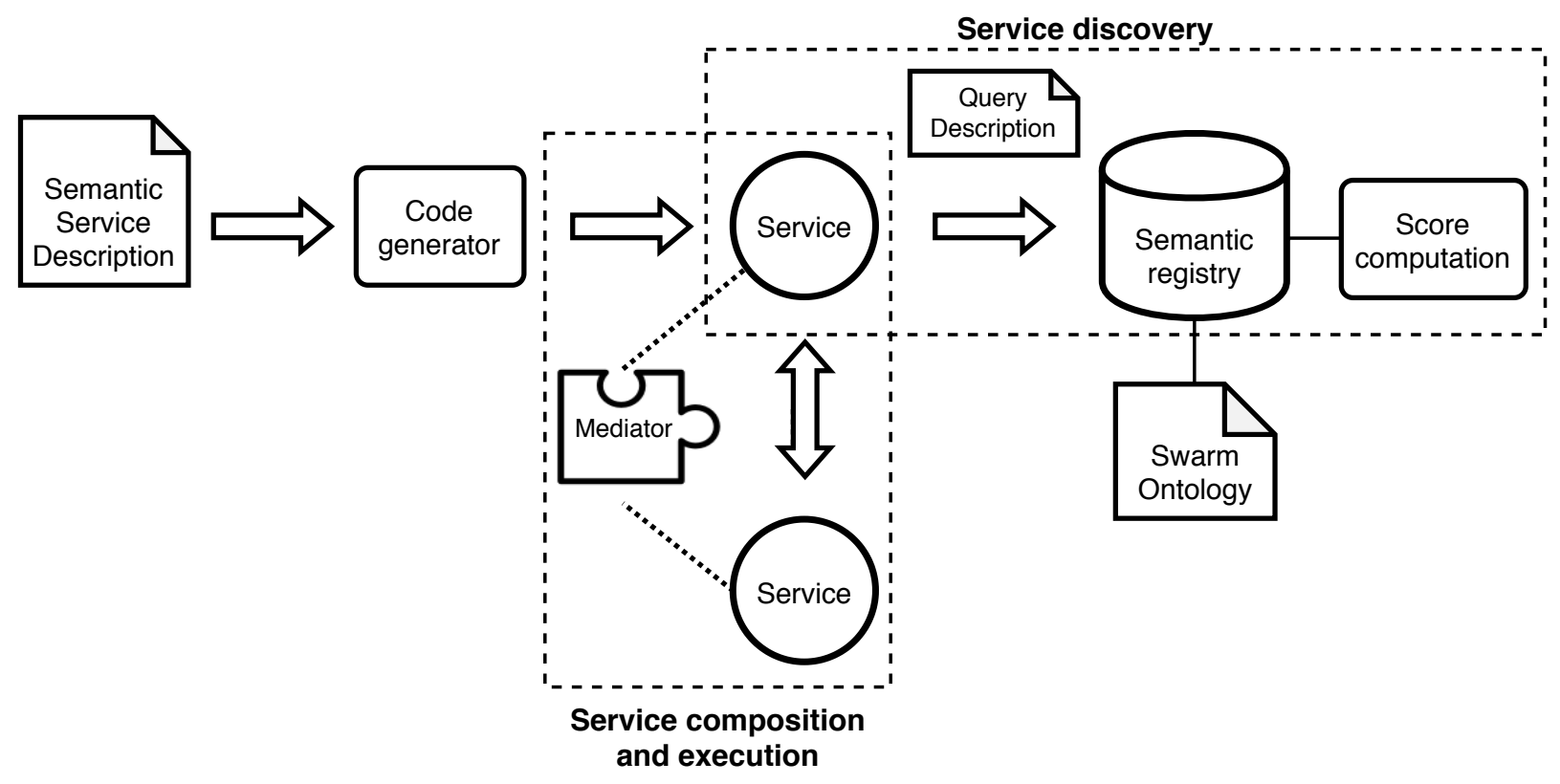

Figure 3.2: Main components of the proposed framework.

\subsubsection{Service description}

A simple human-readable file that contains essential service information, including the provided operations. This file contains semantic information about the service types and exchanged data. Writing this file does not require previous knowledge about semantics from the service creator. Additionally, this file permits the developer to declaratively define a list of services to be discovered and composed. This service description model is presented in detail in Section 4.1. 


\subsubsection{Service discovery}

The process of searching a service in the network, given expected functional and non-functional properties, such as geolocation, input and output type, service and operation type, price, and reputation.

\subsubsection{Semantic query description}

A simple human-readable file that contains the necessary information to find a service in an IoT network. The format of the Semantic query description is presented in detail in Section 4.3.

\subsubsection{Code generator}

A module that takes a semantic service description file as input and produces a service implementation ready to run in the IoT network, thus facilitating the development of IoT applications. This module is presented in detail in Section 4.7.

\subsubsection{Swarm ontology}

An ontology that models the structure of services in the Swarm network, including operations, geolocation, reputation, and price. This ontology is used as the basis for a full taxonomy of services, and data types, ready to be extended by the community of developers. The Swarm ontology is introduced in Section 4.2

\subsubsection{Semantic registry}

A service that stores and retrieves the description files of other services in the network through the processing of query description files. The Semantic registry is presented in Section 4.5

\subsubsection{Mediator}

A powerful concept inspired on the Adapter pattern (Gamma et al., 1995). Mediator is a special type of service that permits the communication among services with incompatible data types, thus enriching the possibilities of service composition. Several mediators can exist for the same task and are ranked according to their relevance.

\subsubsection{Service execution}

A module responsible for automatically invoking a service based on the service description information with no prior knowledge of the API. This mechanism is explained in Section 4.8. 


\subsubsection{Service composition}

A simple mechanism for declarative service composition, based on operation, input, and output types. The composition of services is enriched by the participation of mediator services, as explained in detail in Section 4.9.

\subsubsection{Score computation}

A ranking is performed to select the best candidates in a service discovery process, based on the score of each candidate against the requested service. The computation of this score considers the relevant information in service and query description models. The complete description of this score is explained in Section 4.6.

\subsection{Discussion}

From the developer point of view, we can summarize the main uses of the framework in Figure 3.3, showing the main use cases, the action required from the developer, and the expected functionality provided by the framework. 


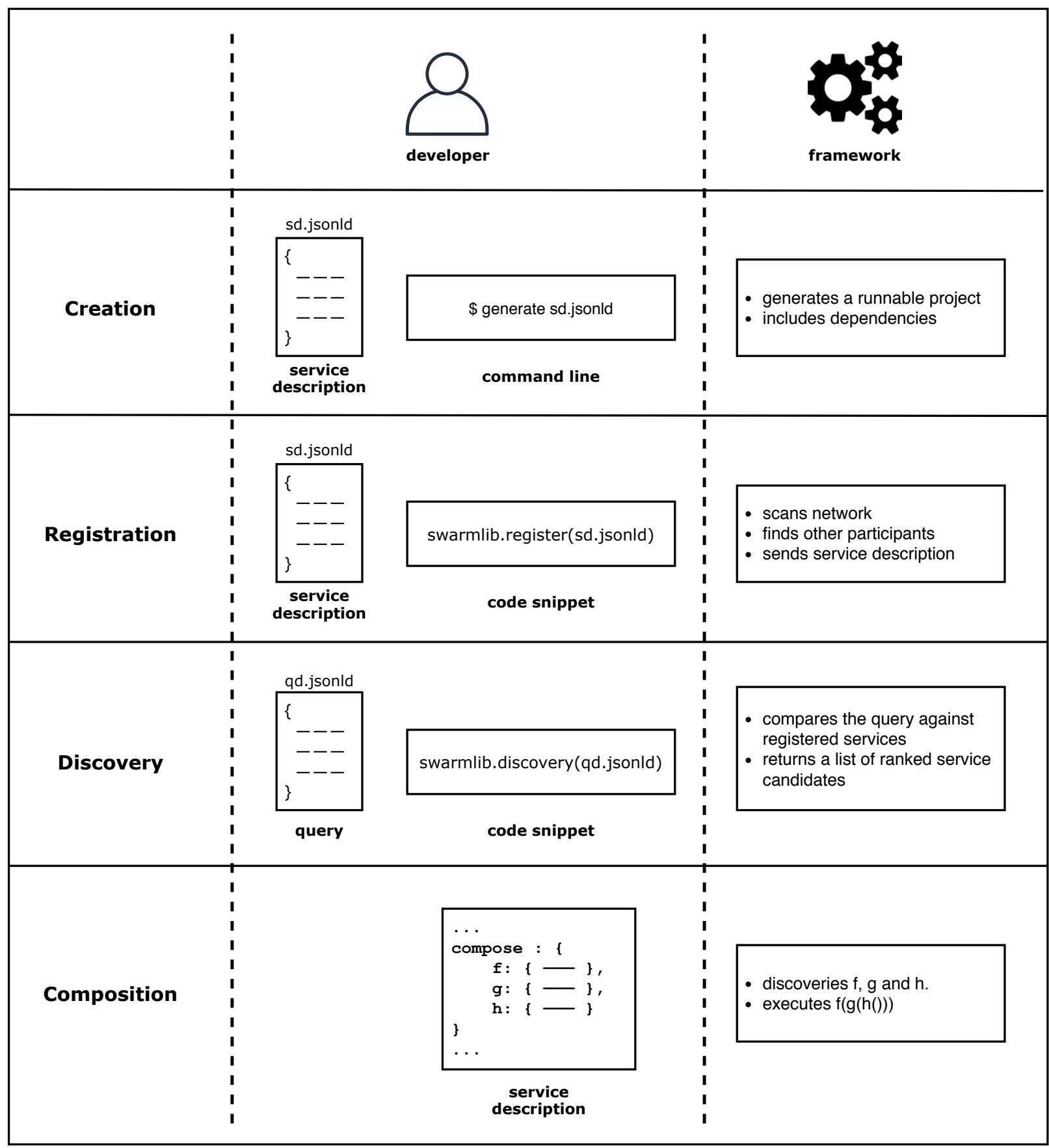

Figure 3.3: Main use cases of the framework from the developer point of view. 
Chapter 4

\section{Framework architecture}

In Chapter 3 we presented a high-level panorama of the framework, describing the main concepts and its relationships. This chapter provides a detailed description of the rationale and functioning of these concepts. An entire section is devoted for each component, explaining the rationale, architecture and examples.

\subsection{Service description model}

A service description file serves to document and advertise the service functionalities in a network. It contains all the information for the service to be discoverable and usable by other software agents. The properties contained in a service description file are usually classified in functional and non-functional. Functional properties are related to the execution of a service and constitute the actual service API, such as endpoint, input, output. All other properties are considered non-functional and serve to specify and constrain the use of the service, including properties such as price, quality of service, and reputation (O'Sullivan et al., 2002). The functional properties of the service are contained in operations section. Accordingly, the non-functional properties of our framework are summarized in Table 4.1, based on the thorough analysis of non-functional properties by O’Sullivan et al. (2005). Thus, geolocation, pricing, reputation and @id, in conjunction with operation serve to fully describe a service in our framework. Regarding the temporal model in Table 4.1, the Swarm access control model, proposed by Fedrecheski et al. (2018) and described in Section 2.1.6, defines a policy-based mechanism to constrain the temporal use of a service. In future versions of this framework, both models will be unified in a single comprehensive block.

\subsubsection{Objectives}

We have identified the following objectives to accomplish through the use of the service description model. 


\begin{tabular}{c|c|c} 
Category & Description & Framework equivalent \\
\hline \hline Locative model & Physical and digital service location & geolocation \\
Service provider & Unique identification of providers & @id \\
Obligations & Mutual service responsibilities & pricing and reputation \\
Temporal model & Availability described in time units. & $\begin{array}{c}\text { In access control model } \\
\text { Service availability }\end{array}$ \\
Combination of locative and temporal & defined on demand by Broker
\end{tabular}

Table 4.1: Non-functional properties categorized by O'Sullivan et al. (2005) compared to our service description model.

1. Advertise the service functionality in an IoT network. That way the service is discoverable and executable by other services in the network. The service description file must be sent to the network and stored in a service directory for further search (Section 4.5).

2. Define a common API for services. Third-party services must be able use this model to describe their operations and interact in the Swarm network through the semantic discovery mechanism (Section 4.4).

3. Facilitate agile service implementation across several platforms. Through the use of a Code generator tool (Section 4.7), a software project for the service must be generated, thus saving development time. Additionally, specific code for different target platforms can be generated.

4. Serve as an API documentation. As the service description model contains the detail of the service operations, including input and output data types, it can be used as a humanreadable documentation, similar to the popular Swagger ${ }^{1}$ model.

5. Permit automatic service invocation. The model must contain the necessary information for the service to be executed without prior knowledge of its API. A similar initiative for documenting APIs is the Hydra Vocabulary ${ }^{2}$ (Lanthaler, 2013), which was proposed to follow the hypermedia as the engine of application state (HATEOAS) principle (Fielding and Taylor, 2002).

6. Declarative service composition. In an initial step towards automatic service composition, we consider the manual description of a service composition, specifying the characteristics

\footnotetext{
${ }^{1}$ https://swagger.io

${ }^{2}$ https://www.hydra-cg.com
} 
of the services that compose the declared service. (Section 4.10).

\subsubsection{Structure}

The structure of the service description model closely follows the Swarm ontology, presented in Section 4.2, thus, it contains the sections: root, operations, pricing, and geolocation. Additionally, as the structure of the service description model is based on JSON-LD (Section 4.1.3), it inherits its basic structure, illustrated in Figure 4.1.

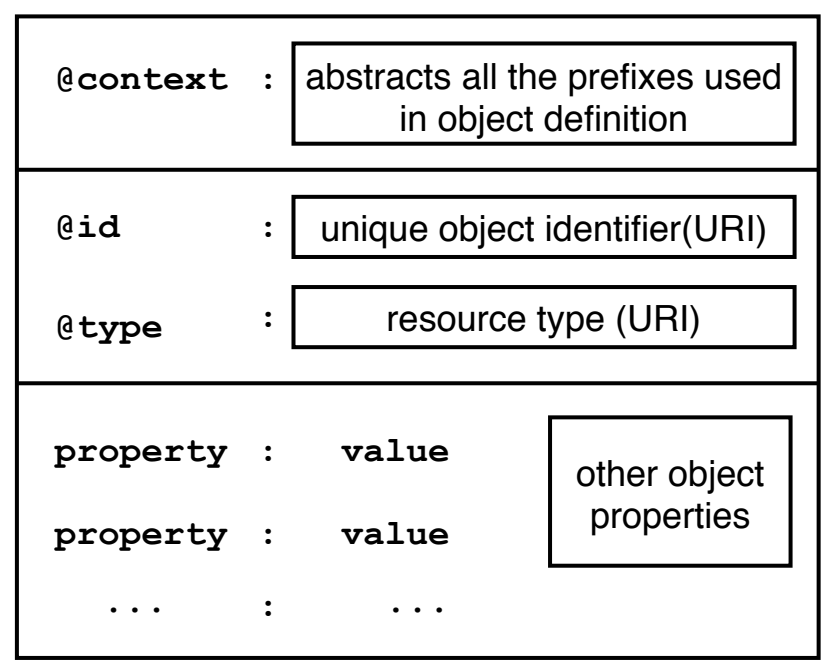

Figure 4.1: Structure of $7 S O N-L D$ object.

\section{- Section root}

The most external section contains general properties related to the service.

- @ context - The @ context field is inherited from JSON-LD format. It can be an URI or an inline object that defines the vocabulary used in the document. The default @context for Swarm services is a link to the Swarm Ontology (Section 4.2), which defines the default concepts used in the Swarm.

- @id - A valid URI that identifies the service in the network. The @id value can be an IP or a resolvable URL that points to the service.

- @type - A valid URI specifying the type of the service. This should inherit the Swarm Service concept in Swarm Ontology.

- broker (URI) - The URL of the Swarm Broker associated to this service (Section 2.1.2). 
- description (text) - A human friendly description of the service.

\section{- Section operations}

Operations are the functional units of the service, that execute atomic actions. This section contains the list of operations that the service provides. Operation information is used for service discovery. Each operation provides the necessary information to be invoked by other agents.

An operation is defined through the following fields:

- @type (URI) - A valid URI specifying the type of the operation. The Swarm Ontology provides four default operations, based on the basic Hydra ${ }^{3}$ (Lanthaler, 2013), which in turn map to the basic functions for persistent storage: CreateOperation, ReadOperation, UpdateOperation, and DeleteOperation (CRUD). When extending those operations, the value of this field must inherit from the hydra:Operation class.

- entry (URL path) - A valid URL path used in conjunction with @id field to identify the address of the service operation for further use (invocation).

- description (text) - Human friendly text that describes the provided operation. This field serves as a documentation for the service API.

- expects (URI) - A valid URI that describes the type of the input parameter. Only one parameter is supported, which must contain all the required information for the service execution. It is equivalent to the body of an HTTP request.

- returns (URI) - A valid URI that describes the type of the operation output. An only response is returned. It is equivalent to the response of an HTTP request.

- method (HTTP method) - A valid HTTP method (GET, POST, UPDATE, DELETE). Following the REST principles it should be chosen according to the semantics of the operation.

\section{- Section pricing}

\footnotetext{
${ }^{3}$ https://www.hydra-cg.com/spec/latest/core/
} 
The pricing section establishes the economic requirements for possible interacting services. This includes price and reputation level.

- minPrice - The minimum price to contract this service, expressed in SwarmCoin.

- maxPrice - The maximum price to contract this service, expressed in SwarmCoin.

- trustThreshold $<0,5>-$ A decimal value between 0 and 5 , which expresses the minimum reputation a requester service must have to contract this service.

- walletId - A unique identification of the payment wallet of the owner of the service. It represents the identity in the blockchain-based structure used for payment and reputation in the Swarm (SwarmChain). The value type is compatible with a Bitcoin address.

\section{- Section geolocation}

This section carries the outdoor location information of the physical device that hosts the service. At this time, our model uses outdoor location, including a single indoor location property (room) for disambiguation if needed. This section and all its fields are optional.

- country - A 2-digit code that represents the country in which the physical device hosting the service is located. This field inherits the addressCountry from Schema ontology.

- city - The name of the city where the device is located. This field is based on to the addressLocality property from Schema ontology.

- state - The name of the state where the device is located. This field is based on to the addressRegion property from Schema ontology.

- postalCode - A postal code. For example, CEP in Brazil. Usually this information helps to more precisely locate the region of an address in the map.

- streetAddress - Based on the property of the same name in Schema

- number - The number of the place in the respective house.

- latitude - The latitude of the device that hosts the service. 
- longitude - The longitude of the device that hosts the service.

- room - This is the only field that shows indoor location, and refers to a simple label to distinguish rooms inside a definite building. The main objective of room is to resolve ambiguity in the outdoor specification of a device location, when based on address.

\subsubsection{Serialization}

Serialization of the service description model consists in transforming the data structure into a format that can be transmitted, stored, and reconstructed to its original structure. Several formats exist to represent an object, such as $\mathrm{XML}^{4}$ and $\mathrm{JSON}^{5}$

The serialization adopted for the service description model is based on the JSON-LD ${ }^{6}$ serialization model, which is in turn based on the popular Javascript Object Notation (JSON) ${ }^{7}$ format. Authors of JSON-LD identified the need to overcome the lack of adoption of various semantic description models, in a phenomenon called semaphobia, due to the complexity of frameworks and serialization formats Lanthaler and Gutl (2011). The JSON-LD serialization model importance is due to the seamless data transformation from a 2-dimensional JSON object (key, value) to a 3-dimensional semantic graph (subject, predicate, object), while maintaining the structure of the initial object.

\subsection{Swarm ontology}

We created the Swarm Ontology in order to model the necessary concepts and relationships used in the framework, such as the service description model, semantic registry module, and ranking algorithm. The ontology was designed as a minimum structure to be extended by developers from the Swarm community, whose structure is depicted in Figure 4.3. The current version of the Swarm Ontology can be found in http://iotswarm.info/swarm-ontology.jsonld

\subsubsection{Structure}

As the Swarm uses a service-oriented architecture (Section 2.1), services have an important role in the framework. We already stated that services conceptually represent the underlying devices

\footnotetext{
${ }^{4}$ https://www.w3.org/TR/xml

${ }^{5} \mathrm{http}: / /$ www.json.org

${ }^{6}$ https://json-ld.org/spec/latest/json-ld/

${ }^{7}$ https://www.json.org/
} 


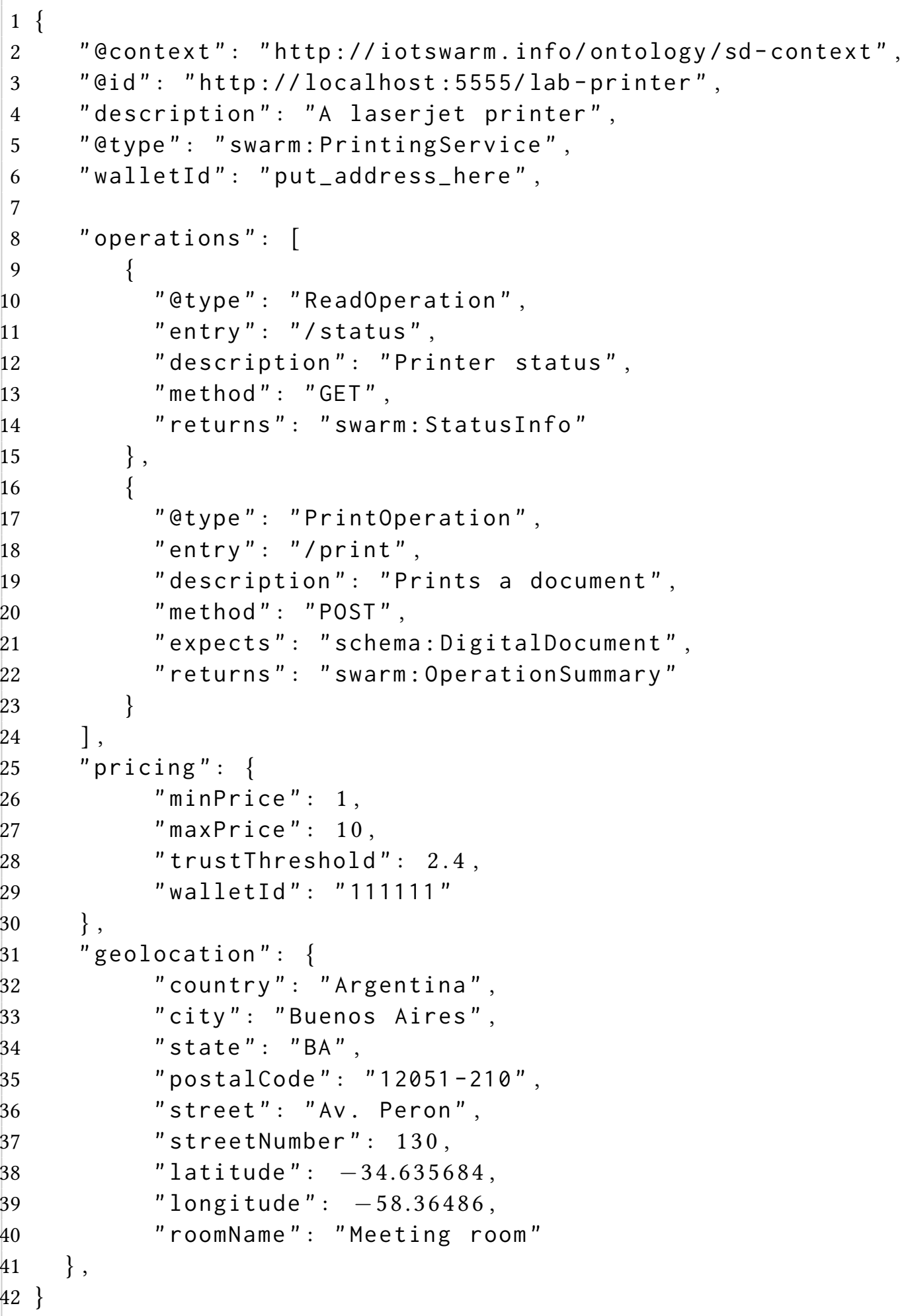

Figure 4.2: Example of a semantic service description. 
in our work (Section 3.1.1). In the Swarm ontology, the entity SwarmService has a cornerstone role, as it defines the structure of the service description model. A SwarmService defines the same properties presented in Section 4.1, and in this section we focus in showing the extension capabilities.

\subsubsection{Extension}

The Swarm Ontology constitutes the base for further development and extension. For this task, new classes can be created using the JSON-LD notation and including them into the @context of the service. Figure 4.3 shows four main points of extension in the Swarm ontology: 1) Swarm Service hierarchy, 2) Operation hierarchy, 3) and 4) Input and Output hierarchy respectively.

SwarmService hierarchy SwarmService is the base class for further services created in the framework. Three essential services are shown in Figure 4.3. Broker represents the Swarm Broker introduced in Section 2.1.2, Semantic Registry is a special service responsible of storing and querying the list of services in the network (Section 3.2.6), and Mediator is a category of services that are used in the mediation mechanism (Section 4.9).

Operation hierarchy The Operation hierarchy is inspired on Operation from Hydra Vocabulary (Lanthaler, 2014). We propose to use the Operation concept with two purposes: to better describe the functionality of a service and to be extended through a hierarchy of operations. We provide four basic operations that map to CRUD (create, read, update and delete) functions, as most services will implement at least these functionalities.

Input and Output The Input and Output properties in Operation constitute the integration point with external ontologies, such as Schema.org ${ }^{8}$, Friend-of-a-Friend ${ }^{9}$, The Music ontology ${ }^{10}$, among others.

\subsection{Query description model}

The query description model is used for finding services in a network. This model is used to filter the services by using the properties defined in the service description model (Section 4.1).

\footnotetext{
${ }^{8} \mathrm{http}: / /$ schema.org

${ }^{9} \mathrm{http}: / /$ www.foaf-project.org/

${ }^{10} \mathrm{http}: / /$ musicontology.com/
} 


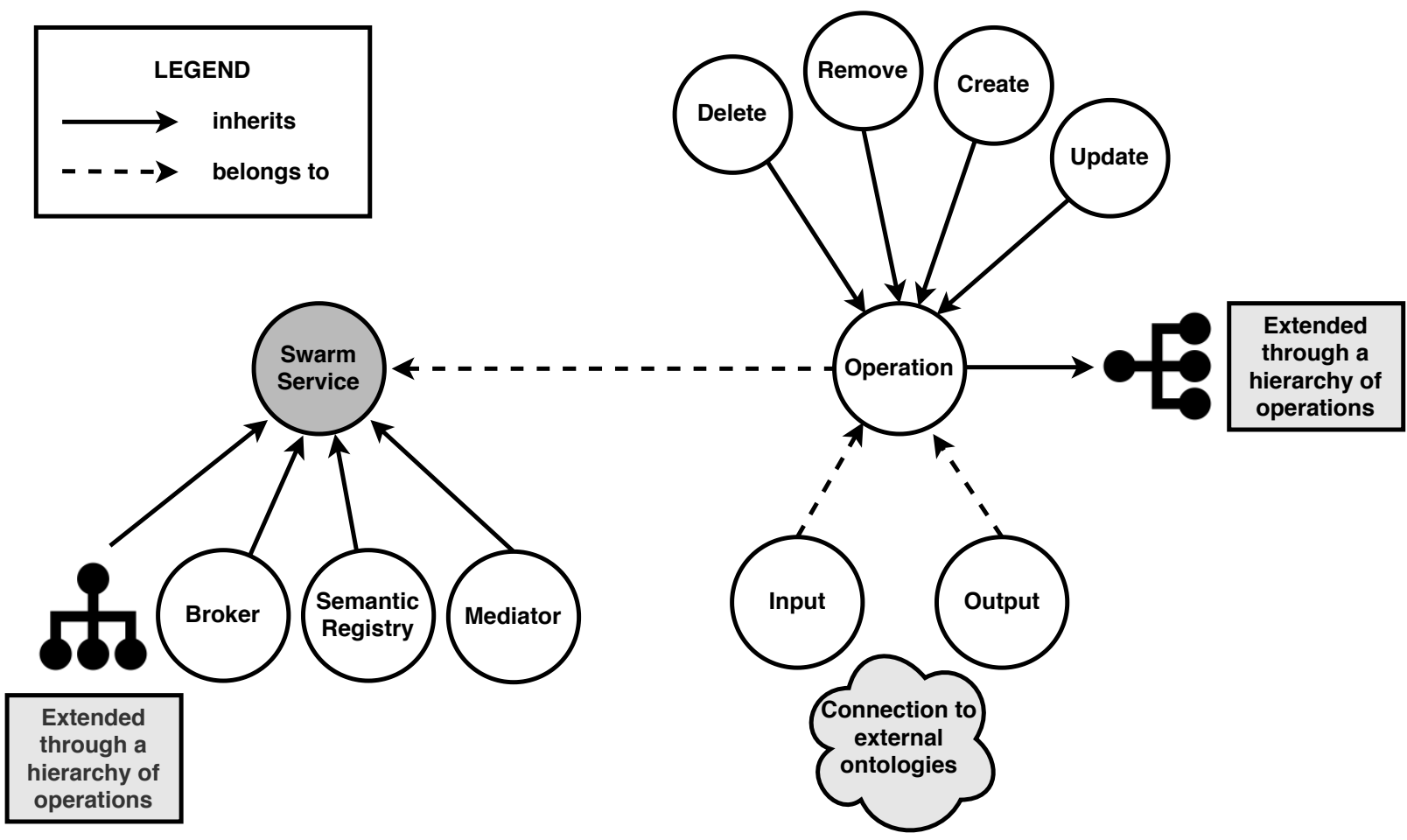

Figure 4.3: Swarm ontology.

\subsubsection{Structure}

The query definition model is divided in three sections that mirror homonymous sections in service description.

\section{- Operation}

- @type - A valid URI describing the type (or subtype) of the service being searched.

The type used here does not need to be specific, and will be searched in a type hierarchy using semantic inference. The exact mechanism of search is explained in Sections 4.4 and 4.5 , while the score obtained by exact or approximate equivalence is presented in Section 4.6.

- expects / returns - Valid URIs that describe the expected input and output of the operation. Similar to operation type, this value can be subtype in the SwarmOntology.

- Geolocation - Fields in this section can be divided in two: address-based and geographicbased. Address-based fields (country, state, city, street, number and postalCode), are not mandatory, although the more detail is given in the query, the more specific the results will be. Geographic-based fields (latitude, longitude and radius) when used, are mandatory 
all three. They serve to describe a circumference with center in latitude/longitude and the given radius of search.

- latitude - The latitude of the location to search. Example: -23.559316

- longitude - The longitude of the location to search. Example: -46.731255

- radius - The radius of the circumference given in meters.

- Pricing - This section serves to filter the candidate services according to the price they charge. This section also includes the trustThreshold, which filters by candidate reputation.

- minPrice / maxPrice Represents the price interval $\left[q_{\min }, q_{\max }\right]$ the requester $q$ is willing to pay for the service. The relationship between the minimum and maximum price of the requester $q$ and the candidate service $s$ guarantees that the expectations of both services are matched: $q_{\min }<s_{\max }$ and $s_{\min }<q_{\max }$.

- trustThreshold - This is the minimum reputation value required for a candidate service to be eligible for interaction. This value is compared to the reputation of the candidate service as defined in Section 4.6.4 (Equation 4.11).

\subsubsection{Serialization}

Analogous to the choose of a serialization format for service description (Section 4.1.3), we use JSON-LD for query description as it is simple, standardized, and does not require to learn a new notation. Figure 4.4 shows an example of this model.

\subsection{Semantic service discovery}

The service discovery mechanism is the main facilitator of interaction in our framework. This mechanism permits two services in the network to find each other in order to start an interaction, which consist in one service (requester) using the functionality of other service (provider). This way, a collaboration network among devices is created. We adopted an approach based on a service oriented architecture (SOA) in our framework, specifically RESTful Web services (Fielding and Taylor, 2002)) as it provides a full set of open protocols and standards to build on top. These are the same principles the Swarm project followed to adopt such architecture.

The most important criterion to find a service in its functionality, as this guarantees the requester 


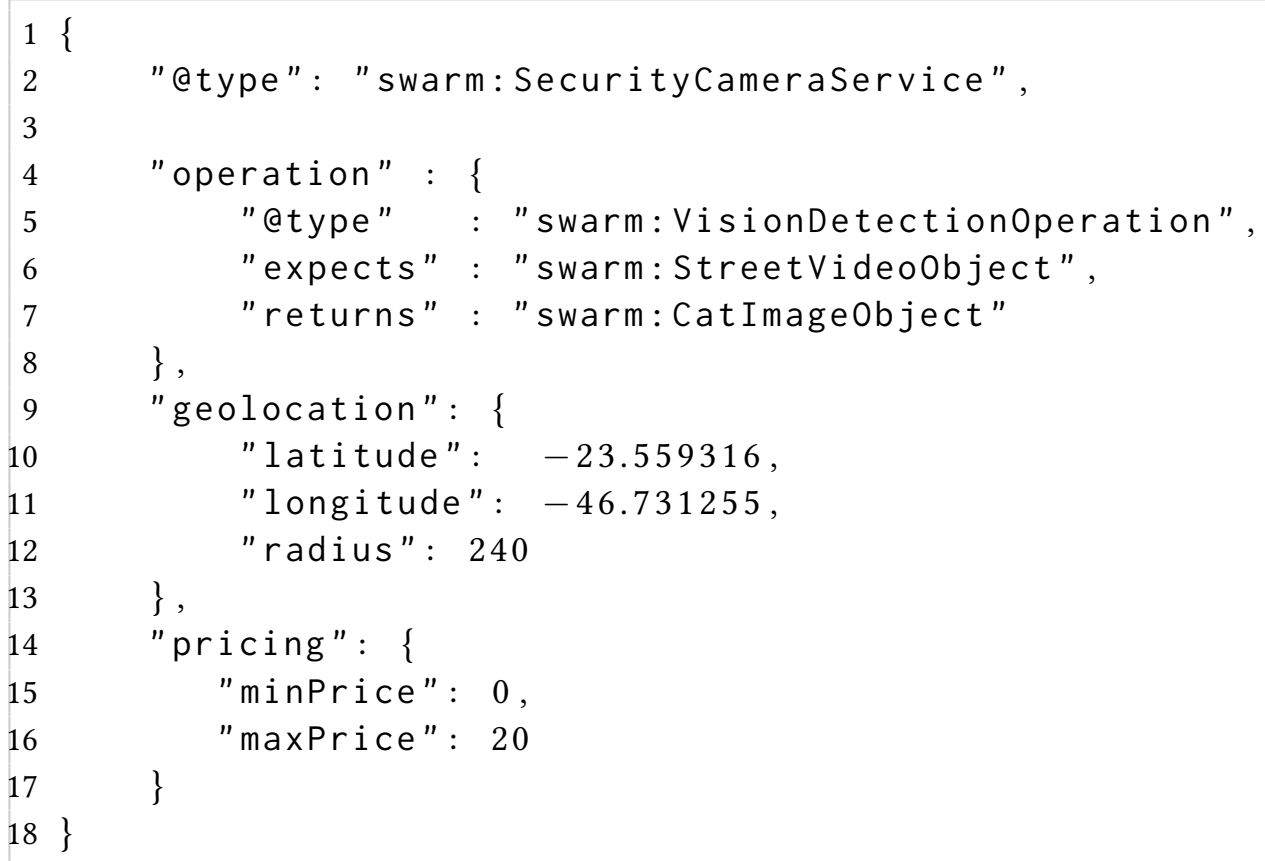

Figure 4.4: Example of a semantic query description.

service will get the expected result. In Section 4.1 we described in detail the structure of the service description format, which use the operation concept to describe the functionality of a service. In Table 4.2 we summarize the properties that define the functionality of a service which we use for discovery. As we explained in Section 4.2 the @type, expects, and returns properties of an operation generate a full taxonomy of types related by equivalence, subsumption, intersection, and disjunction. We recognize the search of a specific type in this graph of types as an ontological problem, as described by Von Rosing et al. (2014).

\begin{tabular}{l|l|l} 
& Property & Description \\
\hline \hline service & @type & service type \\
\hline \multirow{3}{*}{ operation } & $\begin{array}{l}\text { @type } \\
\text { expects } \\
\text { returns }\end{array}$ & $\begin{array}{l}\text { operation type } \\
\text { input data type } \\
\text { output data type }\end{array}$
\end{tabular}

Table 4.2: Properties describing the functionality of a service.

\section{Stages of discovery}

In a previous work (Calcina-Ccori et al., 2018) we proposed the semantic discovery mechanism for the Swarm, which can be generalized for any IoT network. In Figure 4.5 we show the stages of semantic service discovery of our framework. 
1. Register - All the participating services must register in the network at the beginning of its lifetime, in order to be discoverable. To register, a service sends its own description in the format defined in Section 4.3 to a registry. In the Swarm, this registration is mediated by the Broker component, which acts as a local storage, and also sends those descriptions to the semantic registry service. The Swarm network uses an opportunistic policy, so that when semantic registry service is not available, the broker will act as a registry, performing a non-semantic (syntactic) register based on the exact matching of types.

2. Service request - Service request is the trigger of interaction among services. In this stage, the requester service initiates the discovery process by sending a query description file containing the properties described in Section 4.3. In the Swarm network, the query file is sent to the associated broker component, which implements the policies about spreading the request over neighbor brokers and the semantic registry service.

3. Locate - Broker sends the query file to a semantic registry (Section 4.5) service present in the network. Additionally, the broker component stores the query file internally for local search and also redirects the query to other brokers.

4. Selection - In this stage the broker receives a list of candidate services ranked by the score computation detailed in Section 4.6. Broker then tries to establish a contract with the best candidate. In case of failure in the process of negotiation, the next candidate is used.

5. Invocation - In this stage, the information about the best candidate arrives to initial requester service, which has all the information necessary to invoke the service. 


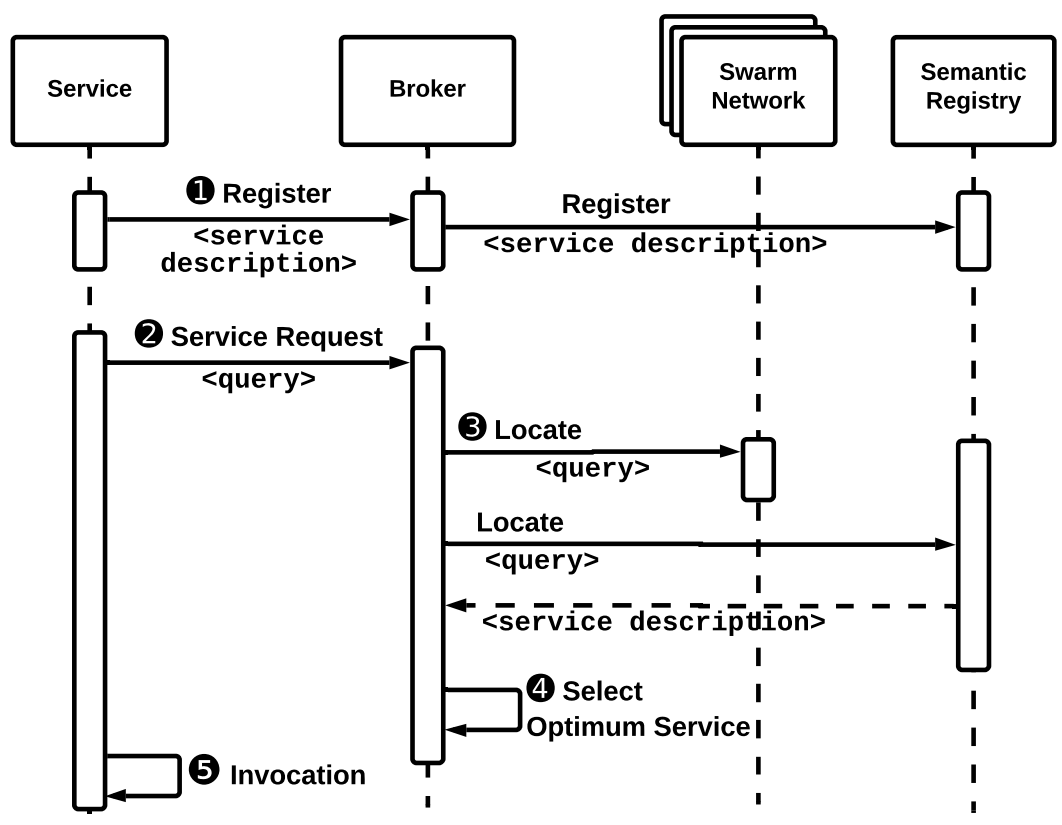

Figure 4.5: Sequence diagram of the semantic service discovery.

\subsection{Semantic registry}

The semantic registry service is one of the core modules of the framework and its functionalities can be summarized as follows:

- Stores the service description files of devices in the IoT network.

- Performs a semantic-based search in the service repository.

- Provides a score for service candidates.

The architecture of this module is shown in Figure 4.6. Next, we describe the main components of this module.

\subsubsection{RDF generator}

This component takes a semantic service description as input and translates it into an RDF graph. This module also imports additional ontologies used in the service definition, by taking advantage of the structure of a JSON-LD file, which separates all the namespace definitions under the @context property. We created an algorithm that imports the referenced ontologies and creates an equivalent RDF graph. The algorithm is based on the JsonLD-TO-RDF procedure from official jsonld-lib software library, and adds a recursive layer for this purpose, which is shown in 


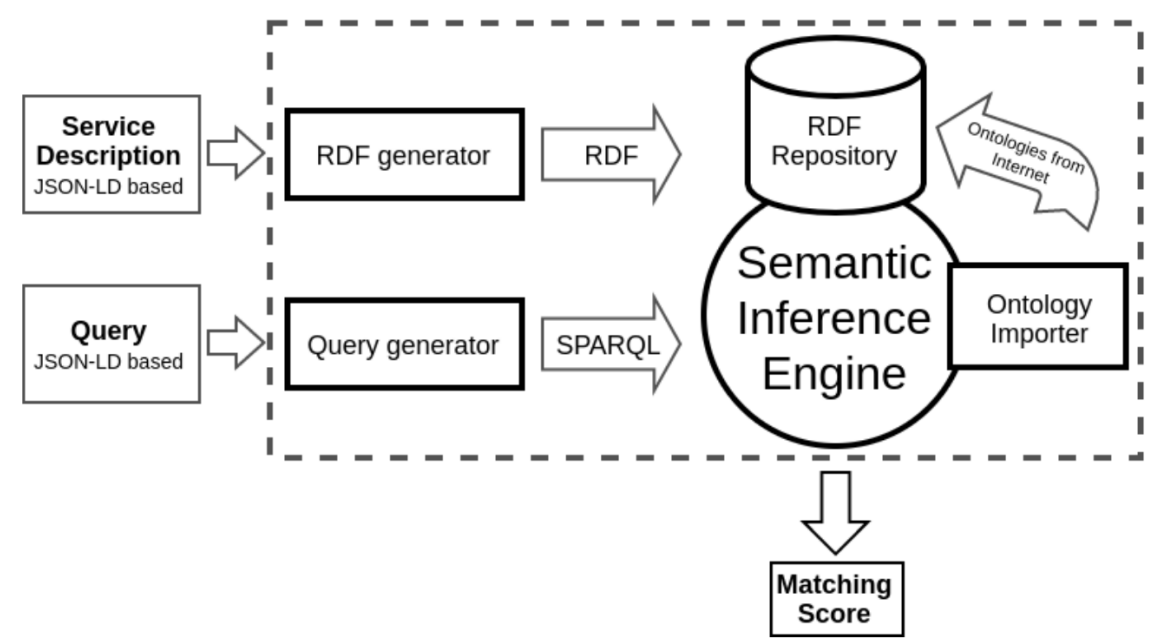

Figure 4.6: Architecture of the Semantic Registry service.

Algorithm 1.

\subsubsection{Query generator}

The query generator component takes a query description file (Section 4.3) as input and produces an equivalent SPARQL query that is sent to semantic inference module. In Appendix A we show an example of a generated query from this module.

\subsubsection{Semantic inference}

This module is the responsible for providing an inference engine that execute SPARQL queries and produces a list of candidate services based on the computed score. To implement this module we created a layer of interation with the Apache Jena Fuseki server ${ }^{11}$, which in turn encapsulates the Apache Jena ${ }^{12}$ inference engine.

\subsection{Score computation}

To classify candidate services in the service discovery process we use a score based on functional and non-functional properties of services. The score of candidate services is calculated according to a combination of the following parameters shown in Table 4.3. Service, operation, input and output type correspond to functional properties of the service and are ranked according to the degree of matching between candidate and query description. The other non-functional properties can be classified in two types: geolocation score measures the proximity of the candidate and queried service and is dependant on the properties of the underlying physical device. Finally,

\footnotetext{
${ }^{11}$ https://jena.apache.org/documentation/fuseki2/

${ }^{12}$ https://jena.apache.org/
} 


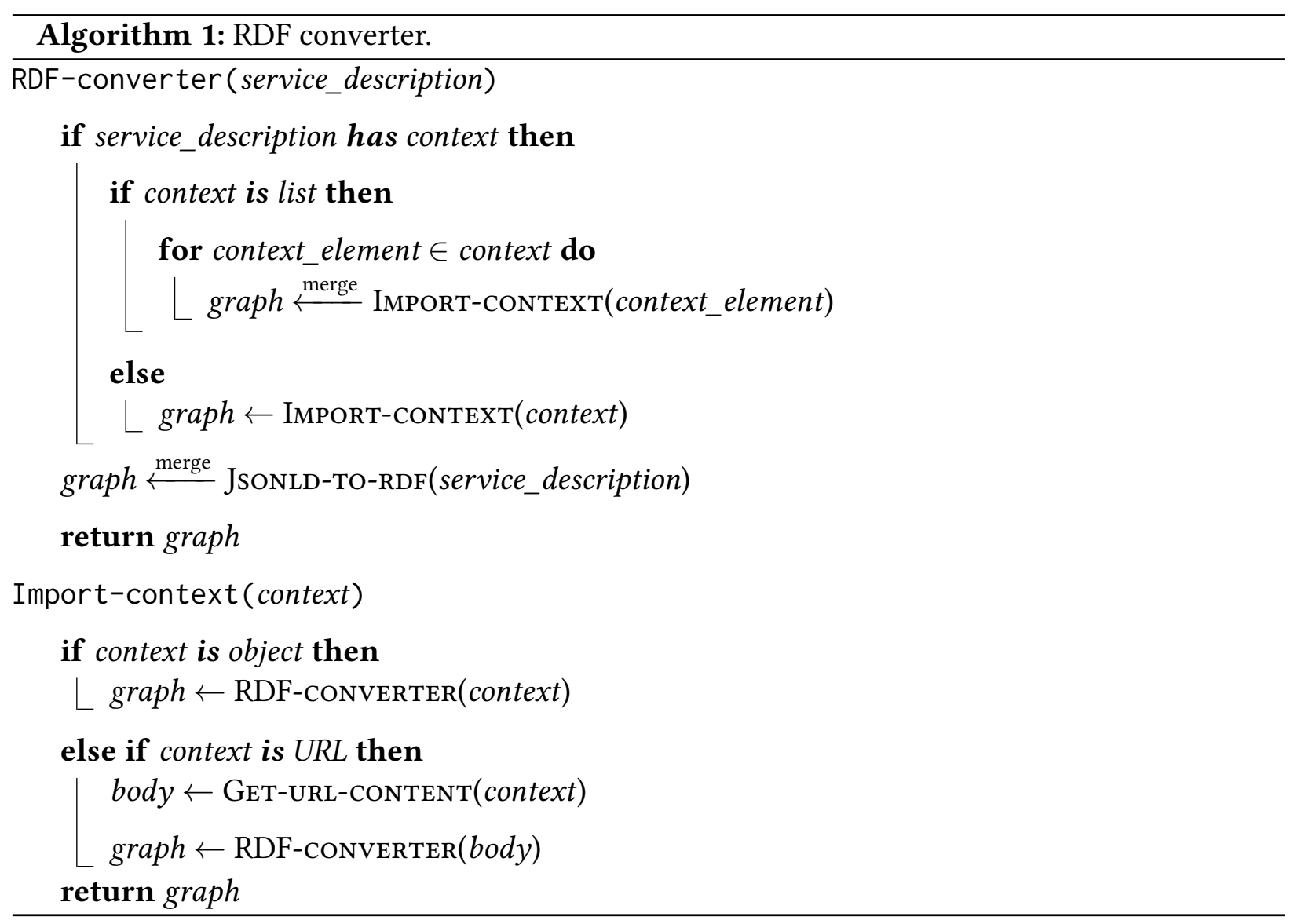

reputation and price constitute the social aspect of the score computation, as the accumulated reputation of a service in the network depends on its score of previous interactions, and this value influences in the service price.

\begin{tabular}{|c|c|}
\hline Parameter & Category \\
\hline $\begin{array}{c}\text { Service type } \\
\text { Operation type } \\
\text { Input type } \\
\text { Output type }\end{array}$ & Functional properties \\
\hline Geolocation & Cyber-physical systems \\
\hline $\begin{array}{c}\text { Reputation } \\
\text { Price }\end{array}$ & Social network \\
\hline
\end{tabular}

Table 4.3: Parameters used to compute the score for candidate services.

\subsubsection{Type equivalence score}

This score is based on the degree of semantic equivalence between types of candidate and query service properties. The same computation formula applies to service, operation, input, and output 
properties. In a prior work we explored the use of semantic equivalence in the Swarm platform, showing how it is possible to create a punctuation according to the degree of semantic equivalence between types (Costa et al., 2015a).

To design the type equivalence of score we adapted the matchmaking algorithm by Li and Horrocks (2004), that considers five matching possibilities for two types $A$ and $B$, summarized in Table 4.4 .

\begin{tabular}{|c|l|c|}
\hline Name & Description & Representation \\
\hline \hline Exact matching & Both types are equivalent & $A \equiv B$ \\
Subsumption & All members of $A$ are also $B$. & $A \sqsupseteq B$ \\
Disjoint & No common members & $S \sqcap Q \equiv \perp$ \\
Plugin & All members of $B$ are also $A$. & $A \sqsubseteq B$ \\
Intersection & There are common members in A and B & $A \sqcap B$ \\
\hline
\end{tabular}

Table 4.4: Matching degrees between concepts.

In our score we consider exact, subsumption, and disjoint matching. In Table 4.5 we show the score matrix applied to compare the types from a candidate service $S$ and the queried service $Q$.

\begin{tabular}{|c|c|c|c|c|}
\hline & Service & Operation & Input & Output \\
\hline \hline Exact & 1 & 1 & 1 & 1 \\
Subsumption & 0.5 & 0.5 & 0.5 & 0.5 \\
Disjoint & 0 & 0 & 0 & 0 \\
\hline \hline Weight & 1 & 1 & 1 & 1 \\
\hline
\end{tabular}

Table 4.5: Partial score matrix for type matching.

From Table 4.5 we define the score for type matching for each of the criteria: service, operation, input, output.

$$
\text { score }_{k}= \begin{cases}\text { score }[\text { exact }][k] & \text { if } S \equiv Q \\ \text { score }[\text { subsumption }][k] & \text { if } S \sqsupseteq Q \\ \text { score }[\text { disjoint }][k] & \text { if } S \sqcap Q \equiv \perp\end{cases}
$$

for $k=\{$ service, operation, input,output $\}$ 


\subsubsection{Geolocation score}

Geolocation score belongs to the interval $[0,1]$. The maximum value of 1 is attributed when the requested latitude and longitude exactly coincide with the candidate service. The score 0 , corresponds to a candidate service outside the search radius.

In previous work (Calcina-Ccori et al., 2019a,b) we introduced the location-aware discovery mechanism, including the ontology and the integration with the Swarm platform. Here we explain in detail the score rationale. First, we define the following variables:

- latitude $e_{s}$ and longitude $_{s}$ : the latitude and longitude of the candidate service

- latitude $_{q}$ and longitude $_{q}$ : the latitude and longitude specified in the query.

- distance $_{q}$ : the search distance defined in the query.

- $R$ : the Earth radius $(6371 \mathrm{~km})$,

We first convert the specified distance $_{q}$ to a compatible search radius, using the sphere equation:

$$
\text { radius }=\frac{180}{\pi} \cdot \frac{\text { distance }_{q}}{R}
$$

Then, we use Equations 4.3 and 4.4 to filter the candidates inside the search radius.

$$
\begin{array}{r}
\mid \text { latitude }_{s}-\text { latitude }_{q} \mid \leq \text { radius } \\
\mid \text { longitude }_{s}-\text { longitude }_{q} \mid \leq \text { radius }
\end{array}
$$

In order to create a score for latitude that maximizes when the distance is minimum, we start from Equation 4.3 to get the expression in Equation 4.5.

$$
\begin{array}{r}
0 \leq \frac{\mid \text { latitude }_{s}-\text { latitude }_{q} \mid}{\text { radius }} \leq 1 \\
0 \leq 1-\frac{\mid \text { latitude }_{s}-\text { latitude }_{q} \mid}{\text { radius }} \leq 1
\end{array}
$$

From Equation 4.5 we mirror this result for the longitude computation and define the latitude and longitude:

$$
\begin{aligned}
& \text { score }_{\text {lat }}=1-\frac{\mid \text { latitude }_{s}-\text { latitude }_{q} \mid}{\text { radius }} \\
& \text { score }_{\text {lon }}=1-\frac{\mid \text { longitude }_{s}-\text { longitude }_{q} \mid}{\text { radius }}
\end{aligned}
$$

Then, we combine the partial scores defined in Equations 4.6 and 4.7 to compute the total score 
for geolocation. Due to the absence of a proper square root function in SPARQL query language, we adapted the computation of the score to avoid the use of that function. We finally define the geolocation score in Equation 4.8.

$$
\begin{gathered}
0 \leq \text { score }_{\text {lat }} \leq 1 \\
0 \leq \text { score }_{\text {lat }}^{2} \leq 1 \\
0 \leq \text { score }_{\text {lat }}^{2}+\text { score }_{\text {lon }}^{2} \leq 2 \\
\text { score }_{\text {geo }}=\frac{\text { score }_{\text {lat }}^{2}+\text { score }_{\text {lon }}^{2}}{2}
\end{gathered}
$$

\subsubsection{Price}

In a previous work we created an economic model for the Swarm (De Biase et al., 2018b), which includes a formula to compute the price of using a service, based on the work by Bogliolo et al. (2012). In this framework we define the price $P(p, c)$ of a transaction between two services: provider $p$ and consumer $c$. Additionally, the price a service $s$ asks for its use belongs to the interval $\left[P_{\min }(s), P_{\max }(s)\right]$, depending on the reputation $R(q)$ of the requester $q$, and on the reputation threshold $R_{t h}(s)$ defined by $s$. In Equation 4.9 candidate service $s$ that acts as the potential provider, and a requester service $q$ has the role of consumer.

$$
P(s, q)= \begin{cases}P_{\text {min }}(s)+\left(1-\frac{R(q)}{R_{t h}(s)}\right)\left[P_{\text {max }}(s)-P_{t h}(s)\right], & R(q)<R_{t h}(s) \\ P_{\text {min }}(s), & R(q) \geq R_{t h}(s)\end{cases}
$$

Price normalization. In order to produce a normalized score candidate service prices in a discovery process, we use a simple normalization formula (Equation 4.10) for $s_{i} \in$ [Candidate service list].

$$
\text { score }_{\text {price }}=P^{*}\left(s_{i}, q\right)=\frac{P\left(s_{i}, q\right)}{\max \left(P\left(s_{i}, q\right)\right)}
$$

\subsubsection{Reputation score}

Similar to the price calculated in Section 4.6.3, we defined the computation of reputation after the $k$-th interaction of service $s R_{k}(s)$ for a service $s$, which is defined as the average feedback $F$ of all previous transactions during its lifetime (De Biase et al., 2018b). When a transaction between two services $s$ and $s^{\prime}$ finishes, service $s$ receives two feedback scores: $F\left(s, s^{\prime}\right)$ from service $s^{\prime}$ and 
$F\left(s, s_{b}^{\prime}\right)$ from the broker of service $s^{\prime}$. Conversely, service $s^{\prime}$ receives scores $F\left(s^{\prime}, s\right)$ and $F\left(s^{\prime}, s_{b}\right)$. The value of feedback $F$ belongs to the interval $[0,0.5]$ and, by definition, all services enter the network with maximum reputation $R_{0}(s)=1$.

$$
\text { score }_{\text {reputation }}=R_{k}(s)=\frac{1}{k} \sum_{i=1}^{k} R_{i-1}(s) F_{i}\left(s, s^{\prime}\right)+\left[1-R_{i-1}(s)\right] F_{i}\left(s, s_{b}^{\prime}\right)
$$

The value of reputation is stored in a Blockchain structure, in order to guarantee the soundness of its value.

\subsubsection{Combined score}

In Equations 4.1, 4.8, 4.10, and 4.11 we defined the partial scores used to define a combined score for all the criteria in set $C=\{$ service, operation, expects, returns, geo, price, reputation $\}$. For each criterion $c \in C$ there is a score $_{c}$ and an associated weight. The latter serves to give priority to a specific criterion in the computation of the combined score, which is defined in Equation 4.12 .

$$
\text { score }=\frac{\sum \text { score }_{c} \times \text { weight }}{\text { c }}
$$

\subsection{Code generation module}

The code generation module was created to reduce the effort of creating new services for the Swarm, by using a declarative notation. The main objectives for the definition of these module are listed below.

- The agile creation of Swarm services from a declarative service description.

- Support for several hardware platforms and programming languages.

- Democratize the development of IoT applications, by reducing the complexity of getting a service ready to run in a real IoT network.

In an early work (Calcina-Ccori et al., 2017) we explored the idea of code generation from a declarative service description, nonetheless, that initial effort was based on the Swagger ${ }^{13}$ project for API description, which lacks support for the use of semantic data types. In a recent work

\footnotetext{
${ }^{13}$ https://swagger.io/
} 
(Calcina-Ccori et al., 2020) we re-engineered the previous work to take advantage of the current semantic service description, presented in Section 4.1.

\subsubsection{Structure}

In Figure 4.7 we show the high-level architecture of the module, whose components are described below.

Service Description Parser. This component is responsible for parsing the service description file, particularly the sections operations and root. From the parsed structure, an internal representation is created to be used by the Service Generator component.

Dependency resolver. This component analyses and determines the language specific packages necessary to execute the project. It also uses a repository of template files for specific target platforms.

Service Generator. This component merges the outcomes of components above and produces a project structure for the specified target, whose structure is described below.

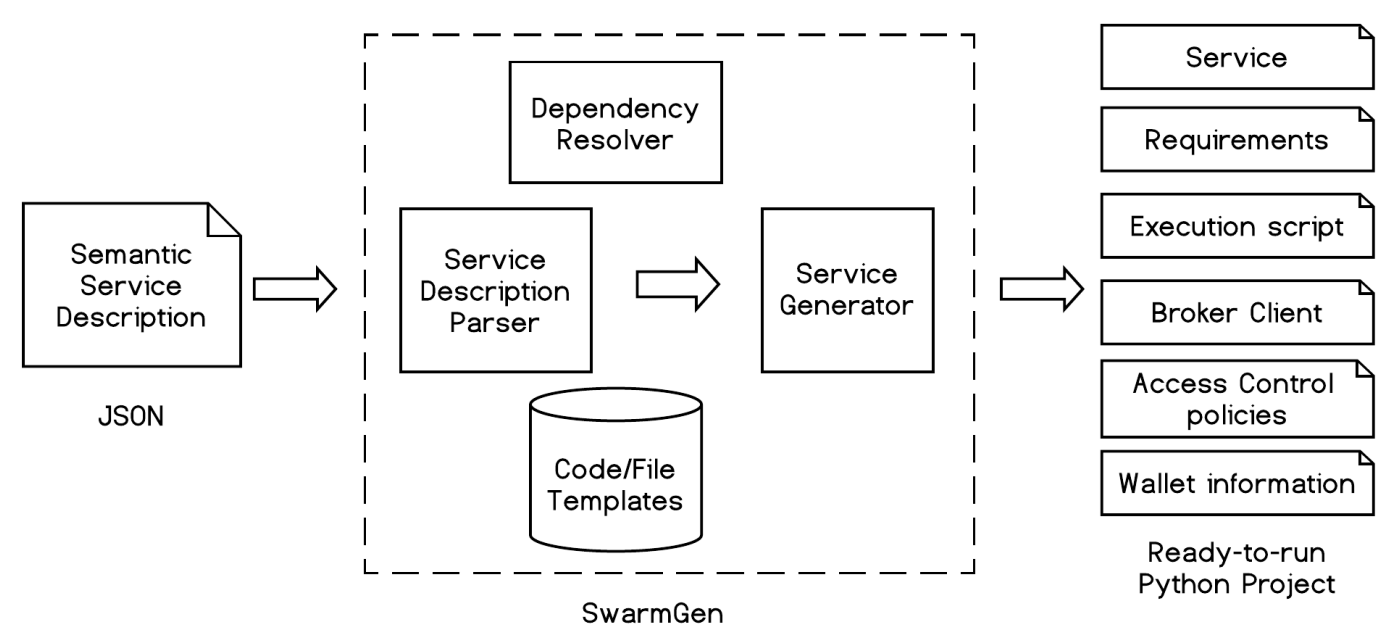

Figure 4.7: Architecture of the Swarm code generator.

\subsubsection{Outcome}

Service. It represents the executable file of the project, that contains the Web service class ready to be used in the Swarm. Spaces for the business logic implementation are indicated with comments. 
Requirements. This file contains the output of dependency resolver with the specification of dependencies, according to the target platform.

Execution script It automates common tasks of the project, such as compilation, test and execution of the service, according to the target platform.

Broker Client. This is software component that encapsulates the communication between the service and the Swarm network, through the Swarm Broker component (Section 2.1.2). The interaction represented in steps (1) (registry) and (2) (service request) from Figure 4.5.

The Broker Client module provides an API to establish this communication among service and broker. Additionally, it provides a method to perform a service level agreement (SLA), and contracting the service provider, including payment and evaluation.

Access Control policies. This file contains the default policies to access the service, as explained in Section 2.1.6 The access control policies create rules based on triples (object, permission, subject), which determine which service or group of services can access a specific operation.

Wallet information. That file contains the private and public keys used by the service to sign transactions with other services. As explained in Section 2.1.3, a transaction represents the interaction between a service consumer and service provider, through the payment for the service use and attribution of a reputation score at the end of the transaction. Both payment and reputation are stored in a Blockchain structure.

\subsection{Service invocation}

The purpose of the automatic execution module is to eliminate the need of writing specific code for invoking a service. Thus, used in conjunction with the discovery mechanism, the only requirements for a developer is the description of the target service to be found in the network. The service invocation mechanism is responsible for performing step 5 from Figure 4.5. After the service candidate is selected, the service description information is used to invoke the service.

The service invocation module decouples the specification of a searched service from the actual implementation of its consumption. This is a necessary step for a future implementation of automatic service composition, as arbitrary services can be composed without the need of writing 
specific code to invoke them, in advance.

Figure 4.8 shows the conjoint use of service invocation and service discovery modules. The effort of the developer to have the desired response relies on writing a query description and specifying the input, thus avoiding the need of knowing the details of the API in advance.

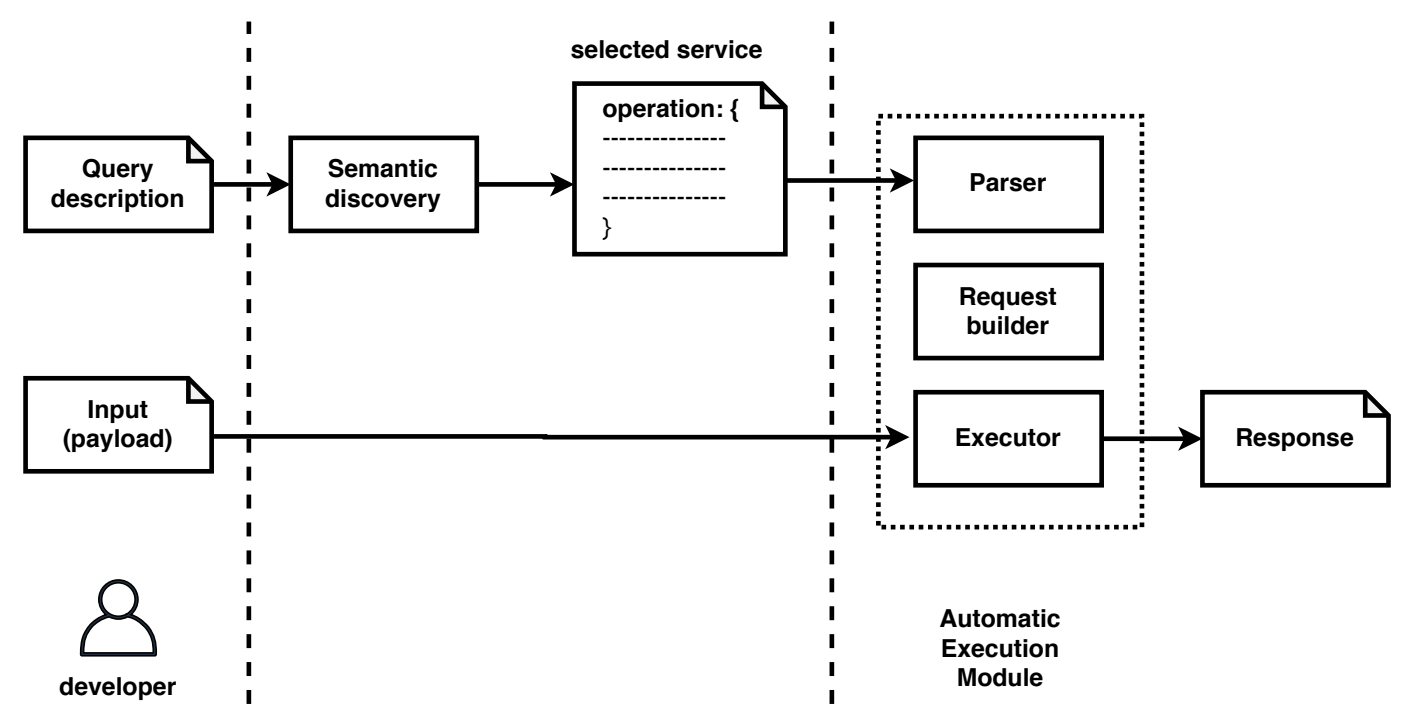

Figure 4.8: Functioning of Service invocation module.

\subsection{Mediation mechanism}

The mediation mechanism consists in using an intermediary service that converts the output of a service $A\left(A_{\text {range }}\right)$ into the input of another service $B\left(B_{\text {domain }}\right)$. Consider a composition plan expressed as:

$$
\begin{array}{r}
A:\left\{o p_{A}, i n_{A}, \text { out }_{\mathbf{A}}\right\} \\
B:\left\{\text { op }_{B}, \text { out }_{\mathbf{A}}, \text { out }_{B}\right\}
\end{array}
$$

so that output $=B(A($ input $))$. If no service is found with the characteristics of $B$, then, the mediation mechanism proposes the discovery of the following two services:

$$
\begin{gathered}
M:\left\{\text { op }_{M}, \text { out }_{A}, *\right\} \\
B^{\prime}:\left\{\text { op }_{B}, *, \text { out }_{B}\right\}
\end{gathered}
$$

The mediator service $M$, by definition, only performs a data transformation, so that, the resulting object is equivalent to the original. Regarding service $B^{\prime}$, as it keeps the same operation type $o p_{B}$, and the output type $o u t_{B}$ from service $B$, it is assumed that $B^{\prime}$ is equivalent to $B$, since the main factor to classify the action of an operation is its type. As an example, consider the query 
expression to search services $A$ and $B$ :

$$
\begin{aligned}
& A:\{\text { Grayscale converter, PNG Image, PNG Image }\} \\
& B:\{\text { Object detection, PNG Image, Object Coordinates }\}
\end{aligned}
$$

Then, in the absence of a service with the characteristics of $B$, a new pair of services are searched, following the definition in Equation 4.13. Then, a new pair of services $M$ e $B^{\prime}$ is found:

$$
\begin{aligned}
& M:\{\text { Mediator, PNG Image, JPEG Image }\} \\
& B^{\prime}:\{\text { Object detection, JPEG Image, Object Coordinates }\}
\end{aligned}
$$

Thus, the new composition of services will be $B^{\prime}(M(A($ input $)))$. Figure 4.9 illustrates the example above.

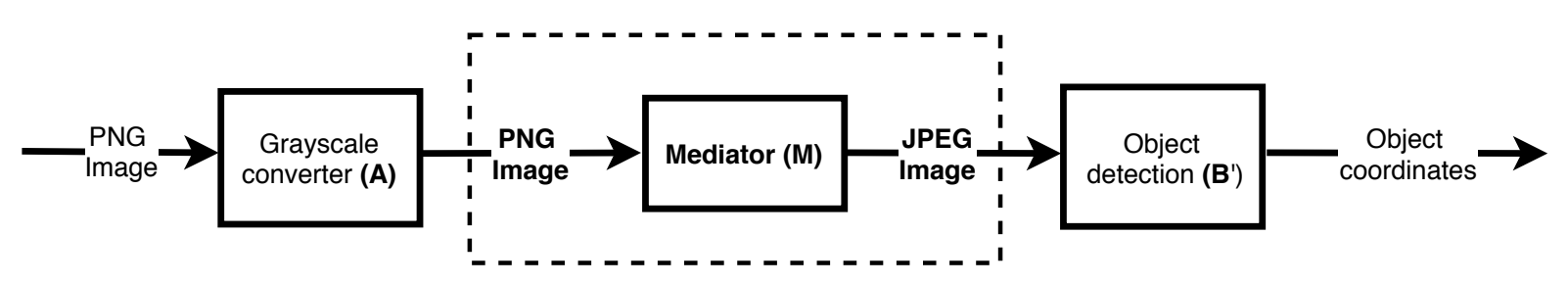

Figure 4.9: An example of the mediation mechanism.

\subsection{Service composition}

The service composition mechanism constitutes the first stage of the automatic composition of service for the Swarm, which will be developed in the short-term (see Section 7.2). At this moment, the service composition concept is closely related to the service creation stage. With the use of a declarative notation for service composition it is possible to define the characteristics of the services that will be searched, executed and composed, without the need of effectively writing code in a specific programming language or target platform. This is very useful for creating services which main functionality is based on the successive execution of other services, as shown in the case study presented in Section 5.2.

Figure 4.10 shows the flow of declarative service composition. The compose field in a service description file contains a list of labeled services to be discovered, specified with the Query description model (Section 4.3). Then, the Code generator tool (Section 4.7) parses the instructions and generates executable code in the selected target. At the end of this process, not only an skeleton of the service file is generated, but the actual business logic code, which consists in a sequencial invocation of the searched services. 


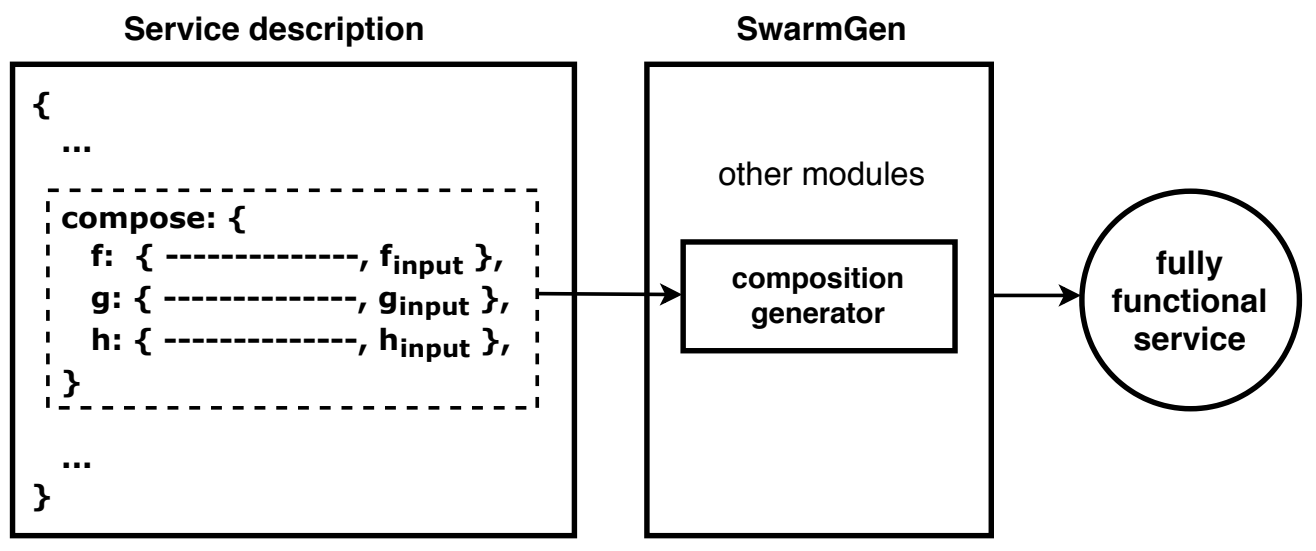

Figure 4.10: Declarative composition of services. 
Chapter 5

\section{Implementation and use case}

In this chapter we describe a reference implementation of the modules introduced in Chapter 4. Additionally, we show the integration points with the Swarm framework and illustrate the use of the proposed framework through a use case applied to an IoT application of surveillance.

\subsection{Implementation}

This reference implementation of the framework was carried out considering the current status of the Swarm platform (Section 2.1). The design decisions of this implementation are based on the Swarm architecture. The main programming language used for the implementation of this framework was Python ${ }^{1}$, based on its versatility; ease to learn, code and debug; availability across different platforms; abundance of libraries; and open source license (Hillar, 2016; Madisetti and Bahga, 2014). Other technologies we use are detailed in the next sections, when explaining the corresponding framework module.

\subsubsection{Code generator}

As the purpose of the code generator is to help programmers to kick-start the development of Swarm Services, we implemented this module in the form of a command line tool that receives a JSON-LD service description as input and produces a ready to run project in the selected target platform. For this reference implementation the initial target is Python 3.0 (and all the platforms that support this version) using the Flask ${ }^{2}$ Web framework (Lokhande et al., 2015).

Generated code. The concrete instances of the generated files for the current implementation are briefly described as follows. The service. py file is a standalone Web service based on Flask. The generated file includes a function definition for each declared operation, with annotations for the corresponding HTTP methods and access control policies, as shown in Figure 5.1.

\footnotetext{
${ }^{1}$ https://www.python.org/

${ }^{2}$ https://palletsprojects.com/p/flask/
} 


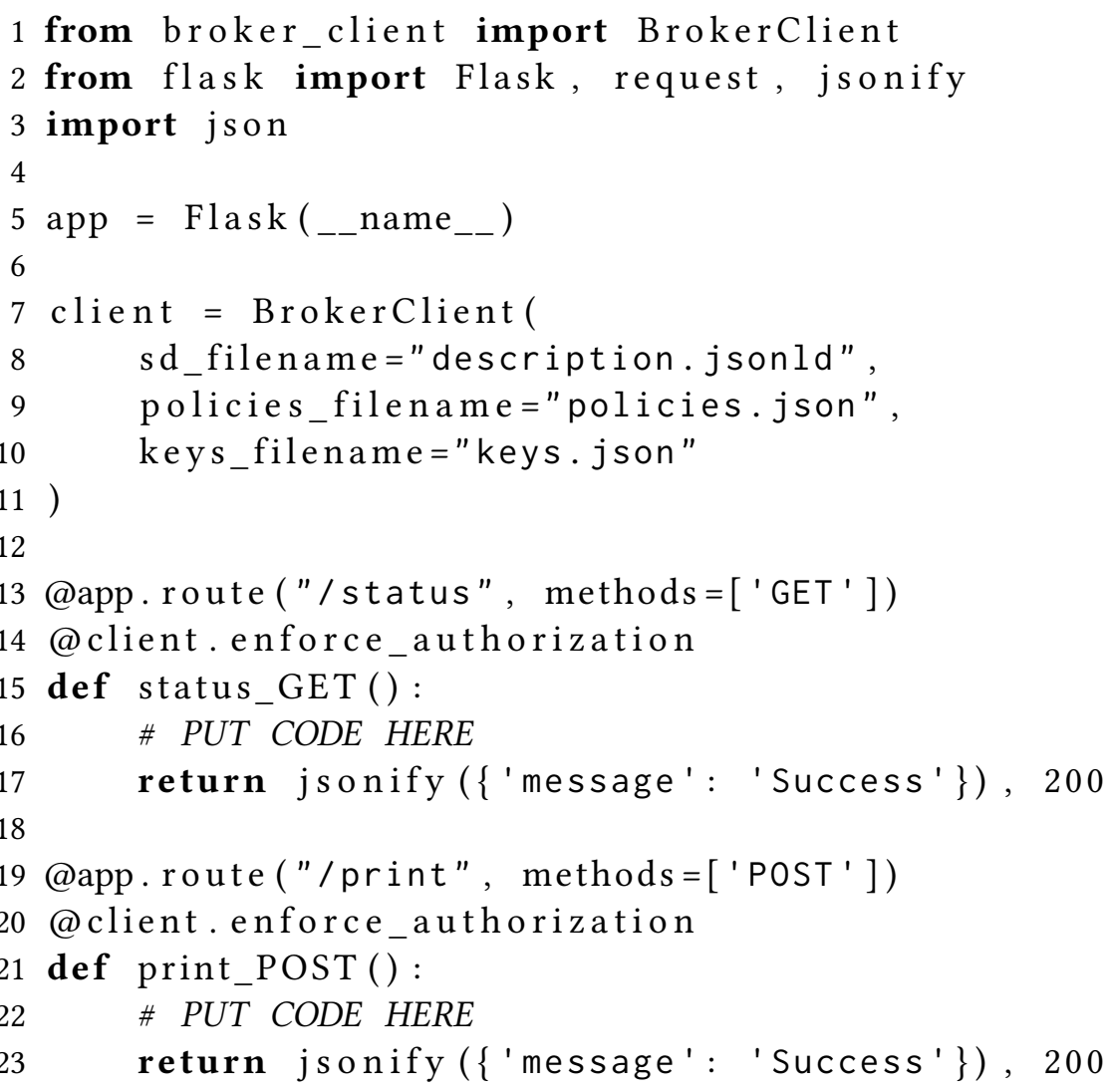

Figure 5.1: Excerpt from the generated service code, showing the operation implementation.

Accordingly, requirements.txt contains the necessary packages to execute the service, imported from the Python Package Index ${ }^{3}$. The execution script corresponds to a properly configured Makefile. The access control policies are written in a triple-based JSON file, whose format is specified in Fedrecheski et al. (2018). Finally, the key.json file stores the private and public key for service, according to De Biase et al. (2018b).

\subsubsection{Semantic registry}

Following the service-oriented architecture of the Swarm, the semantic registry module was implemented as a Web service that offers the registry functionality to the network. At the beginning of its lifetime it registers itself as a special service into the Swarm broker. The semantic registry service provides a REST API that supports locate and register operations. The implementation of the query generator module consists in a set of templates that produce an SPARQL file. An example of the generated file is shown in Appendix A.

\footnotetext{
${ }^{3}$ https://pypi.org
} 


\subsubsection{Swarm ontology}

The Swarm ontology (Section 4.2) provides a basis for integration with further ontologies. From the possible languages to write the ontology, such as RDF-XML, RDF-Turtle, OWL and N3, we selected JSON-LD for two reasons. The first one is that JSON-LD permits an straightforward conversion to RDF, which is the default format the semantic database (Apache Fuseki) accepts. The second reason is the facility to use it in other semantic documents that use JSON-LD, which only requires the inclusion of the respective URL into the @context field. The current version of the Swarm ontology can be found at http://iotswarm.info/swarm-ontology.jsonld and is reproduced in Appendix B.

\subsection{Use case}

In a recent work we presented a use case for the Swarm that gathers resources in an IoT network, in order to identify targets in a surveillance system (De Biase et al., 2020). In this thesis we use the same use case for demonstration the use of our framework.

\subsubsection{Use case description: Lost cat}

This use case aims to find a lost cat in a specific geographic area, by gathering resources that cooperate to build a surveillance system. Figure 5.2 shows an overview of the expected functionality, which is stated below:

A cat has been reported lost from its house, then the owner asks the network to find it, by issuing her personal assistant with the command find my cat, which is analysed and translated into a command find(cat).

The personal assistant will request access to security cameras around the house in order to gather recent images and process them with a computer-vision based detection service, which in turn will detect the presence of a cat in those videos. If cats are identified, the location of the respective cameras is marked in a map, with a photo of the detected target, for user confirmation.

\subsubsection{Participants}

Figure 5.2 illustrates the participants of the use case, which are summarized as follows: 


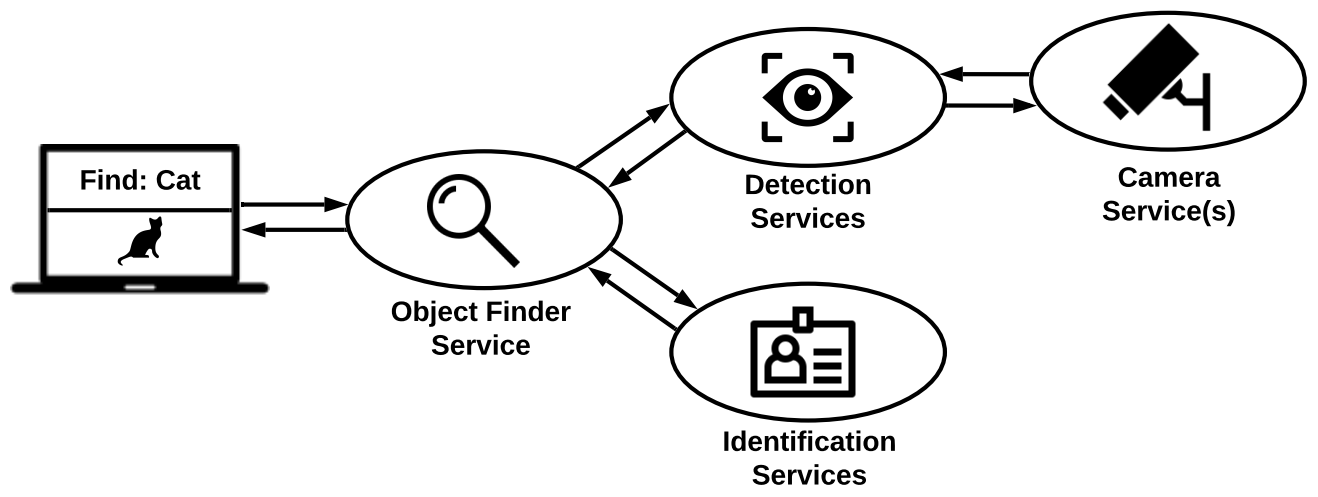

Figure 5.2: Services that participate in the use case.

- Personal assistant - This service has direct communication with the user. The personal assistant receives and maps the user intentions into commands to other services to perform the required task.

- Object finder - This finder is a service responsible for recruiting and combining the functionalities of other services to accomplish the desired task.

- Detection service - This service receives an image and detects a specific target. In this case, it uses a computer vision algorithm specialized on identifying cats in an image.

- Camera service - This service represents a camera that provides images on request.

- Identification services - This service is specialized in identifying if a given cat is the cat being searched.

\subsubsection{Using the framework}

Service description. In this section we will illustrate the service description for the VisionFinder service. Figure 5.3 shows an example of the service description for finding a target from an image.

Service composition. An example of service composition is given in Figure 5.4, where two services are composed through a declarative composition specification defined in Chapter 4.

Ontology. In Figure 5.5 we show an excerpt of the ontology defined for the Lost cat use case. 


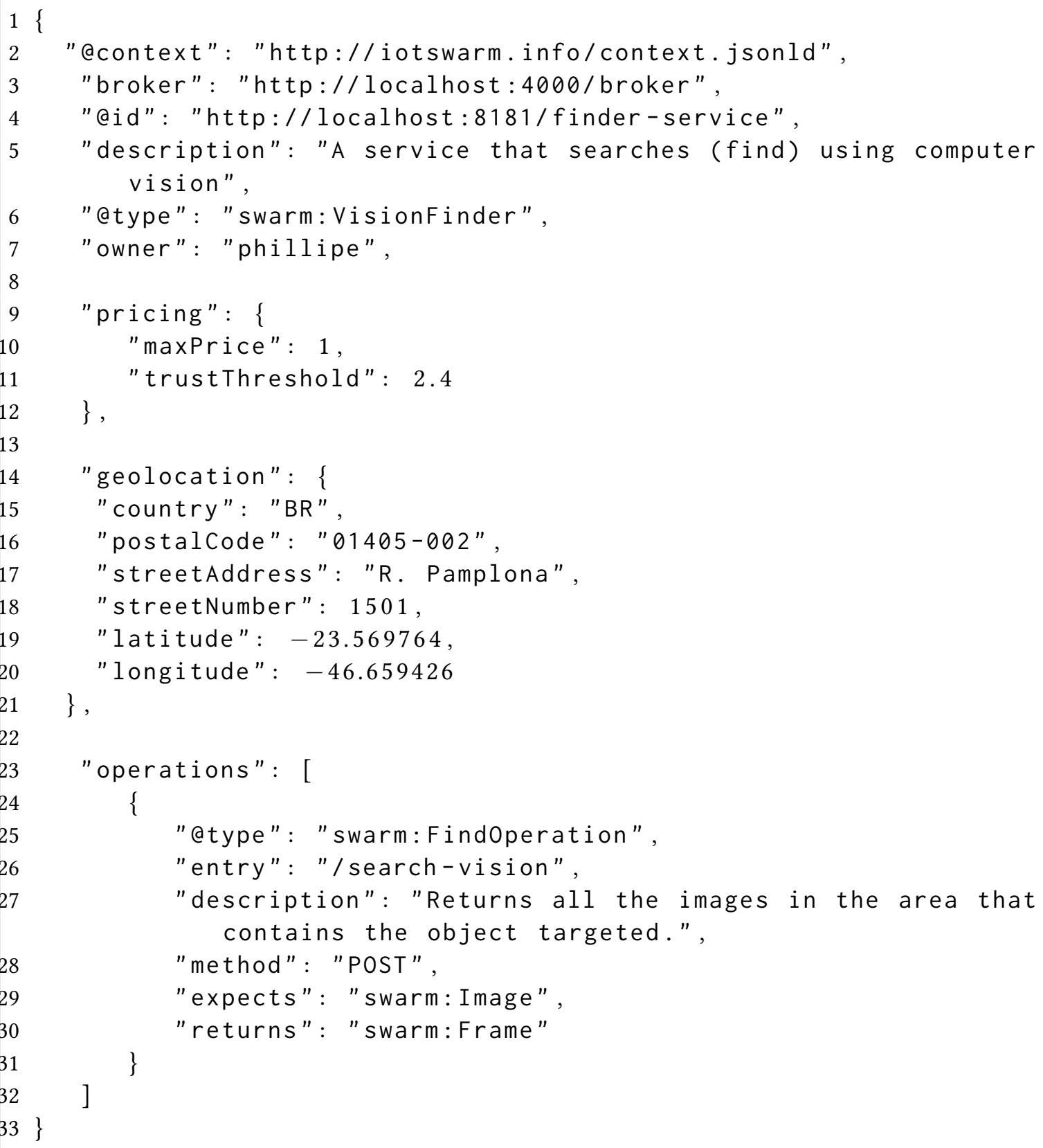

Figure 5.3: Service description of the VisionFinder service. 


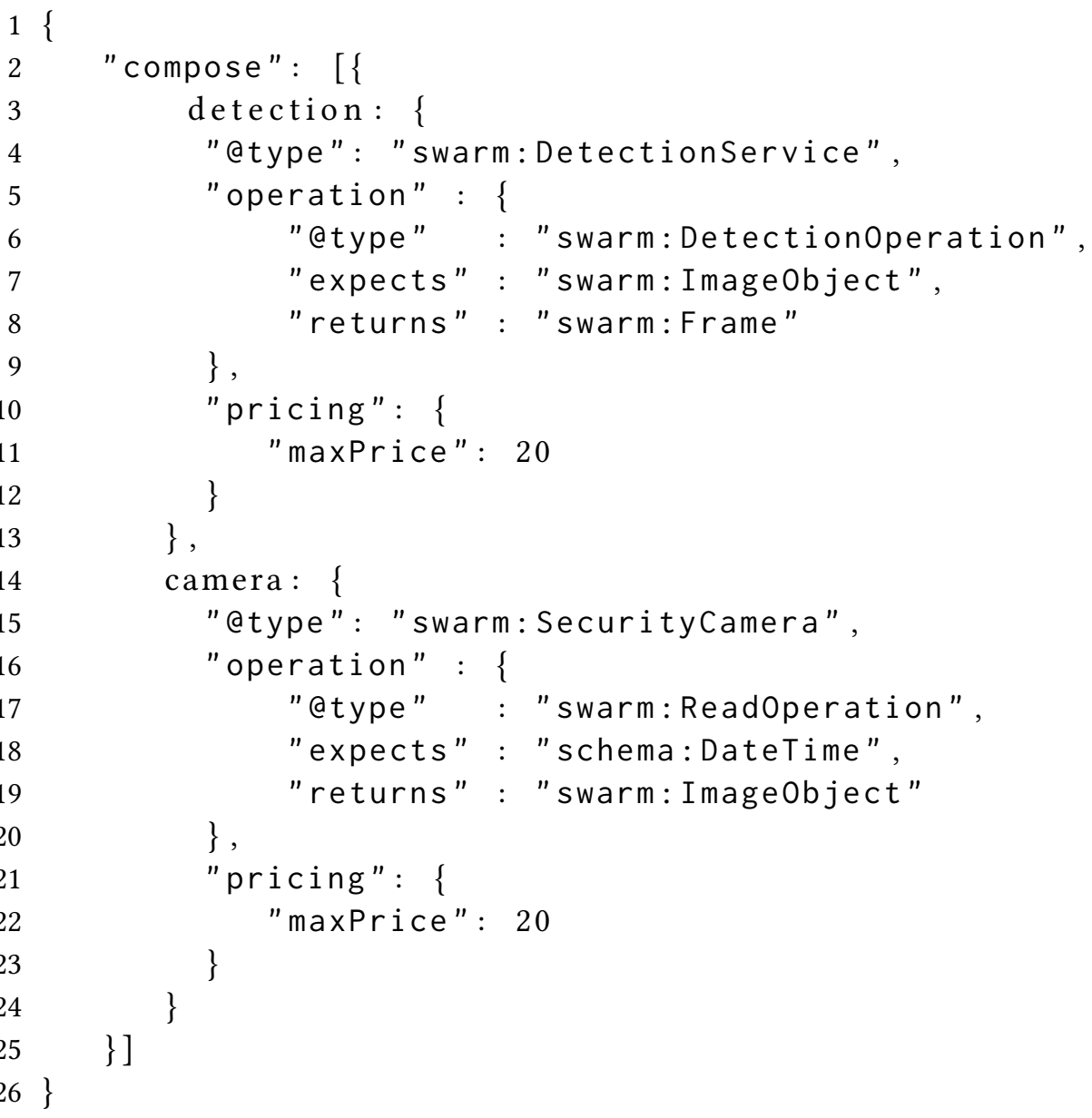

Figure 5.4: Declarative composition of services in the Swarm. 


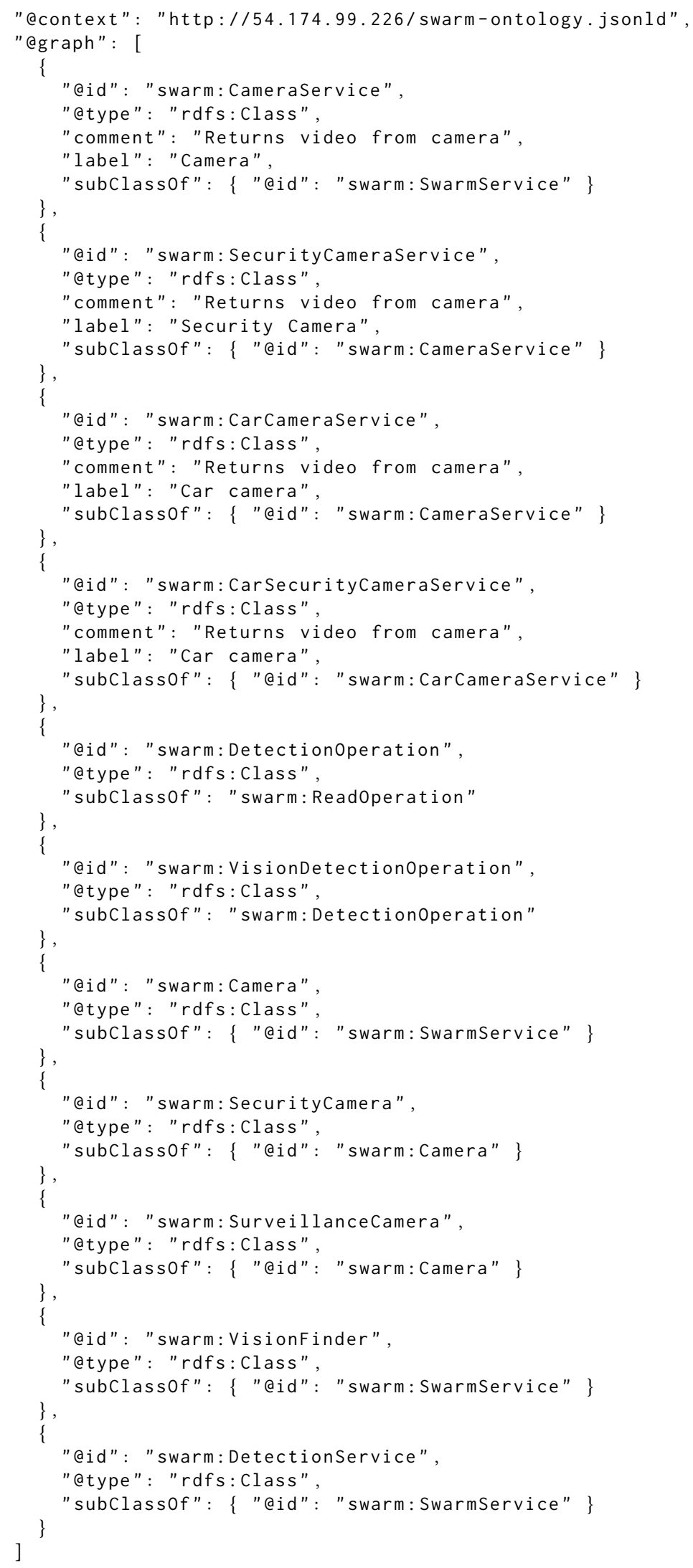

Figure 5.5: Excerpt from the ontology used in the Lost cat use case. 


\section{Results}

The main result of our thesis was the integration of the interaction framework into the Swarm platform, thus contributing to have an IoT platform with a set of components for device interaction.

\subsection{Evaluation}

In order to evaluate our framework we measured three main aspects: programming effort, execution time, and storage space. Additionally, for execution time evaluation, we used two different hardware configurations, that represent a common Internet of Things and Desktop hardware platforms.

- Caninos Labrador ${ }^{1}$ board, with a 64-bit quad-core ARM CPU and 2GB of RAM. This singleboard computer is used in the development of IoT applications.

- Laptop with Quad-core Intel Core i7-6500U CPU and 8GB of RAM. This configuration represents a common configuration for a cloud service.

\subsubsection{Programming effort}

We argue that our framework contributes to the reduction of programming effort to create IoT applications. To measure this we use the number of lines of code as an indicator of programming effort of performing selected task in the creation of an IoT application. To accomplish this, we analyze the use case presented in Section 5.2, and compare the lines of code before and after the adoption of our framework. The selected tasks correspond to the different stages in the creation of the application. Table 6.1 shows the number of lines of code for each task, and Figure 6.1 depicts the relationship between these measurements.

- Service skeleton - The minimum code necessary to create a service capable of registering

\footnotetext{
${ }^{1}$ https://caninosloucos.org/en/labrador-64-en/
} 
in the Swarm network. It includes the implementation of the API and communication with Swarm Broker for registering.

- Service description - The service description model (Section 4.1) is evaluated in terms of the equivalent code necessary to describe the service using semantic annotation. Thus, we compare the description written using our JSON-LD-based model to the equivalent using the RDF format, which is the default notation to describe a semantic object. Although with this comparison we analyze the effort reduction in written code, it is important to note that the reduction of dimension from a triple (subject, predicate, object) to a pair (key, value) greatly contributes to reducing the effort also.

- Query description - Similar to the service description case, we compare the query description model (Section 4.3) to the equivalent raw query written using the SPARQL query language. SPARQL is used to declaratively search triples in a graph of objects.

- Service composition - To measure the effort in writing a service composition list we consider the composition section in service description (Section 4.10) used to declaratively write the services involved. We compare this measure to the actual programming code necessary to write the equivalent task. As a reference we use the same use case with the implementation in Python programming language.

- Service mediation - We compare the effort of creating a service mediation by comparing to the equivalent programming effort without the framework, which includes manually performing two discoveries and matching the input and output parameters, given that the mediator service already exists.

\begin{tabular}{|l|c|c|}
\hline Interaction type & with framework & without framework \\
\hline Service skeleton & 25 & 350 \\
Service description & 25 & 307 \\
Query description & 18 & 94 \\
Service composition & 35 & 70 \\
Service mediation & 0 & 85 \\
\hline
\end{tabular}

Table 6.1: Comparison of lines of code between the different tasks performed in the framework.

By analyzing both Table 6.1 and Figure 6.1 we observe a significant difference between the re- 


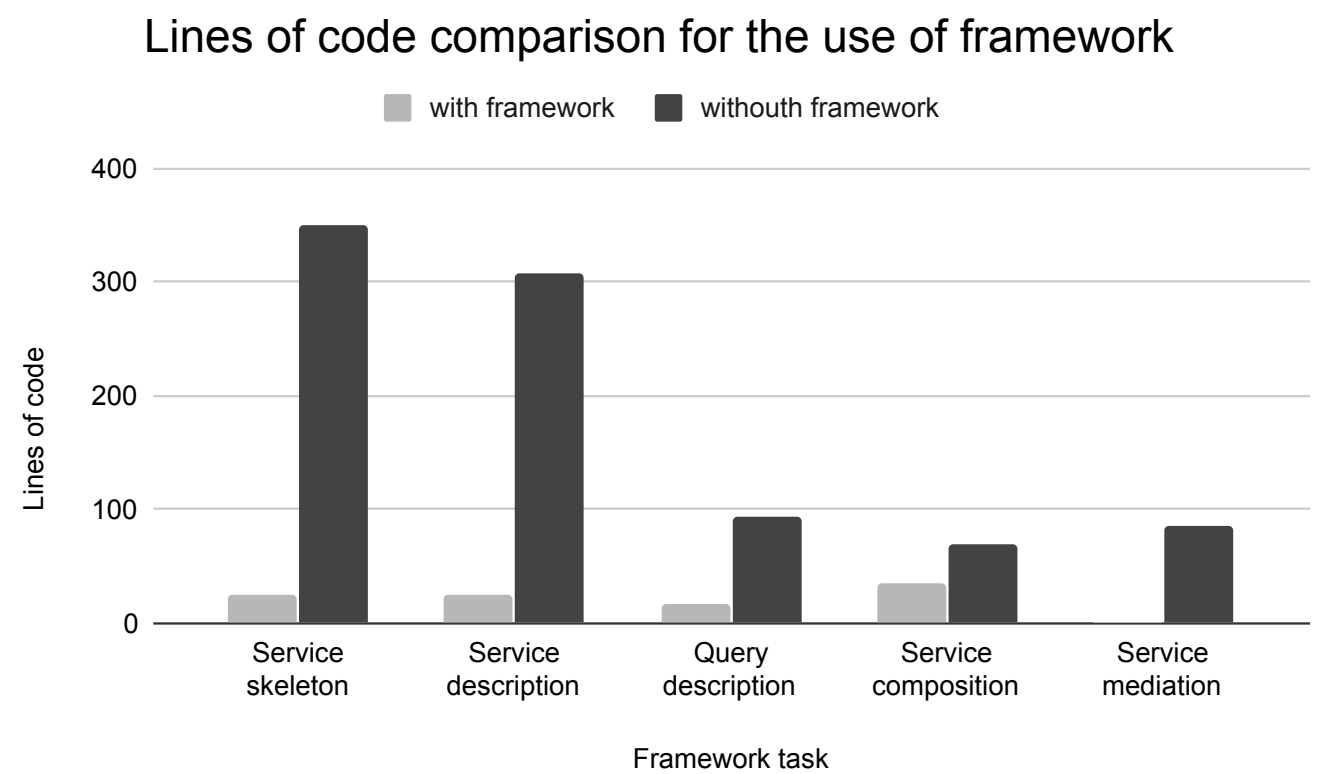

Figure 6.1: Comparison of lines of code for the same application before and after using the framework.

quired lines of code for implementing the same use case, with and without our framework. The approximated ratio for this values is $\frac{906}{103}=8.79$, which we interpret as the factor of reduction of programming effort when using our framework.

\subsubsection{Execution time}

To compare the execution time, we computed the average of 40 executions of different services of our use case in three configurations, as shown in Figure 6.2.

- The first measure, with no semantic registry is when our framework is not used. In this case, a simple storage and recovery is made by the SwarmBroker from an in-memory data structure.

- The second measure correspond to the use of a semantic registry running on an embedded device commonly used for IoT applications. With this configuration the additional overhead is approximately $270 \%$ of the time when the framework is not used.

- In the third measure, semantic registry runs on a more powerful computer, comparable to a cloud service. In this configuration, the additional overhead is about $50 \%$ of the time without using the framework.

In the case of composition, the execution time depends on the amount of composed services, since 
for each one, a discovery process must be performed. Given the computation above, the approximate overhead would grow linearly by a factor of 2.7 (Labrador board) or 0.5 (x86 architecture). In this case, we considered a minimum case of two composed services.

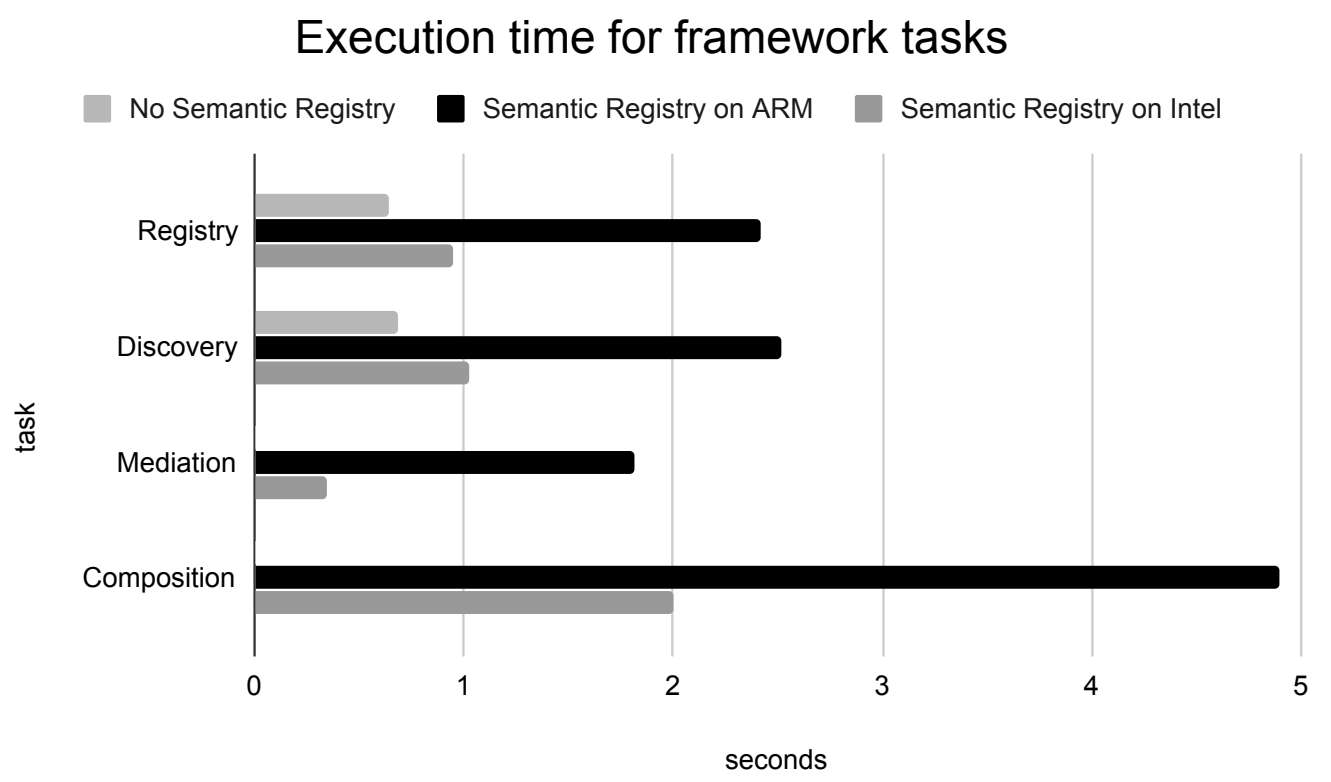

Figure 6.2: Comparison of execution time for tasks with and without the use of the framework.

\subsubsection{Storage}

The computation of the storage needed by our framework strongly depends on the underlying database of choice. In our case, the Apache Jena Fuseki server ${ }^{2}$ stores the complete RDF graph in a set of files that store the actual nodes, prefixes, and indexes. In our implementation we use a single dataset named semantic-registry, which is in the same directory where the semanticregistry service runs. An empty dataset in the Fuseki database uses 201.3 MB. This includes 24 fixed-size files each one starting with 8.4 MB. Those files grow on demand, doubling their size as the number of elements increases. Given that behavior, we were not able to deduce a linear relation between the number of tuples or services and the total size used by the dataset. In our tests, we inserted 10000 services in the dataset and the total size of the database grew up to $211 \mathrm{MB}$, which was mainly due to two files: node2id.dat, which doubled its size to $16.8 \mathrm{MB}$ and nodes. dat, which increased up to $1.6 \mathrm{MB}$ from an initial size of 0 bytes. For this reference implementation, we consider that size is not being excessively large, as it still can run on the Caninos Labrador board, which is a computer targeted for IoT applications.

\footnotetext{
${ }^{2}$ https://jena.apache.org/documentation/fuseki2/
} 


\subsection{Comparison with other works}

In Section 2.3 we presented related frameworks that aim to help in the development of IoT applications, or more generally, Web services, through the inclusion of Semantic Web technologies. In this section we aim to provide a qualitative comparison between our framework and those works. In Table 6.2, we summarize the comparison to these works, that is commented in more detail below.

The main differences between our framework compared to OWL-S and WSMO consist in the adoption of the REST paradigm for Web services. Also, the choice of a format for service and query description in our framework aims to simplify the semantic annotation of services by isolating the complexity into the @context section of our model. This differs from OWL-S, which uses a XML-based notation and WSMO, which uses the frame logic-based WSML language. Further works provided mechanism for automatic service composition and mediation for both projects, as shown in Table 6.2, which are still under development in our framework. Furthermore, in contrast to OWL-S, which provides a rich set of tools, we offer simple but helpful tools for code generation and libraries for service interaction. Additional tools are being developed to facilitate the development of Swarm applications.

Similar to ReLL proposed by Alarcon et al. (2011), we adopted the RESTful service paradigm for our framework and included a static service composition mechanism, although the Petri Nets based composition results in a more sophisticated solution. Besides these two main features, our framework provides additional features, such as code generation, composition, and mediation. Additionally, the use of JSON-LD instead of XML favors the legibility of service description files.

In contrast to the OSF project (Mayer et al., 2017), our framework still does not provide a visualization tool. Also, a feature inspired by the knowledge packs of OSF is to be included into our framework, as it provides an easy way to manage knowledge from different domains. In contrast to our project, the OSF framework is mainly oriented to managing the knowledge base for IoT applications, without going further to service composition and mediation.

Among the related work we considered in this section, the SWoTPAD (Silva et al., 2019) is the one 
of the most recent efforts and embraces RESTful services. One of the main highlights of SWoT$\mathrm{PAD}$ is the implementation of automatic service composition. Although there is an important convergence between the ideas proposed by the SWoTPAD project and our framework, some features are out of the scope of that work, such as the inclusion of Social IoT concepts and the mediation of services. Another key difference between both projects is the service description format. While the emphasis in the Swarm project was in simplicity to reach a wide number of new programmers, the SWoTPADL language provides more powerful features, such as preconditions, postconditions and compAbility constructor for creating composite services.

The Hydra Core Vocabulary (Lanthaler, 2013) constitutes one of the main inspirations to create the service description model proposed in this thesis. Although the Hydra project did not aim to provide a complete framework for Web services, the vision of its creators is to leverage the automatic invocation of RESTful services through the HATEOAS paradigm. The HATEOAS restriction (Hypermedia as the Engine of Application State) defined by Fielding and Taylor (2002) proposes that the application interface (API) is defined dynamically through the successive interaction with a Web resource (service) and depends on the current state of the resource, in contrast to fixed interfaces defined by other protocols such as CORBA IDL. Although that objective is not completely withing the scope of this thesis, the advances of Hydra in creating a common vocabulary for Web APIs are relevant for our work. 


\begin{tabular}{|c|c|c|c|c|c|c|c|c|}
\hline Criteria $\backslash$ Work & Swarm & OWL-S & WSMO & oneM2M & OSF & ReLL & SWoTPAD & Hydra \\
\hline \hline Prog. environment & own tools & - & WSMT & own tools & OSF & - & SWoTPAD & Hydrus \\
Code generation & Yes & - & - & - & - & - & Yes \\
Service description & JSON-LD based & OWL-S & WSML & OWL & RDF & ReLL (XML-based) & SWoTPADL & JSON-LD based \\
Query description & JSON-LD based & SPARQL & WSML & SPARQL & SPARQL & SPARQL & SWoTPADL & - \\
Service composition & Static & Automatic & Yes & & - & - & Static & Automatic \\
Mediation & Restricted & Yes $^{3}$ & Yes & - & - & - & - \\
Ontology & SwarmOntology & OWL-S & WSMO & BaseOntology & & ReLL Model & WSMO-based & Hydra Core \\
Visualization & - & - & WSMX & - & Yes & - & - & - \\
\hline
\end{tabular}

1. For example, the proposed by Sirin et al. (2004) and Klusch et al. (2005)

2. For example, the proposed by Farnaghi and Mansourian (2013)

3. For example, the proposed by Vaculín and Sycara (2007)

Table 6.2: Comparison of the features of our framework with similar works. 
Chapter 7

\section{Conclusions}

\subsection{Synthesis}

In this work we have proposed a framework for device communication in a network of things, such as the Swarm. In Chapters 3 and 4 we presented in detail the rationale of the framework, the main components and its relationship. We described a reference implementation and integration with the Swarm in Chapter 5, and evaluated the results in Chapter 6.

This work serves as a first step towards the creation of an intelligent network that provides automatic cooperation of devices (See Section 7.2). The main contributions of this work are summarized as follows:

- The definition and implementation of a framework for discovery, execution, and composition of services in an Internet of Things network.

- A simple description format for declarative description of semantic services.

- A basic ontology for semantic service description.

- The concept of service mediators, that permit communication among otherwise incompatible services.

- A declarative mechanism for service composition.

- A tool for code generation from declarative service description.

- A ranking mechanism for choosing the most suitable device in an interaction process.

- An ontology for geolocation of devices in an IoT network.

Additionally, during the development of this thesis we successfully produced the following publications: 
- In Semantic Swarm (Costa et al., 2015a), we discussed the use of semantics to help solving the problem of interoperability in the Swarm, identifying key technologies and providing an illustrative example. The results of this initial exploration helped to define the type matching of the ranking algorithm in Section 4.6.1.

- In the two articles The semantic mediation for the Swarm: An adaptable and organic solution for the Internet of Things (De Biase et al., 2017) and Enabling semantic discovery in the Swarm (Calcina-Ccori et al., 2018), we defined the basis for semantic discovery in the Swarm, introduced in Section 4.4. Additionally, we proposed the architecture for the Semantic Registry module, presented in Section 4.5.

- In Agile servient integration with the Swarm: Automatic code generation for nodes in the Internet of Things (Calcina-Ccori et al., 2017) and SwarmGen: a framework for automatic generation of semantic services in an IoT network (Calcina-Ccori et al., 2020), we presented two stages of the development of a code generation tool for services in the Swarm, explained in Section 4.7.

- In the article Swarm economy: a model for transactions in a distributed and organic IoT platform (De Biase et al., 2018b), we introduced a model for price and reputation in the Swarm, used in the ranking algorithm of candidate services, in Sections 4.6.3 and 4.6.4.

- In Describing services geolocation in IoT context (Calcina-Ccori et al., 2019a) and Locationaware discovery of services in the IoT: a Swarm approach (Calcina-Ccori et al., 2019b), we proposed an ontology for geolocation of services in a Swarm network, used in the computation of the geolocation score, in Section 4.6.2.

- In Surveillance Swarm: Gathering resources for finding targets in a distributed global network of smart devices (De Biase et al., 2020), we defined the architecture of a surveillance systems, used a case study of our framework, in Section 5.2. 


\subsection{Future work}

\section{Automatic service composition}

The declarative mechanism we proposed for service composition (Section 4.10) constitute a first step towards a more intelligent network that is capable to self-organize to respond to a user request. The declarative notation proposed in this work will be used as a set of building blocks for the automatic generation of service compositions, based on the analysis of the graph of services in the Swarm network. In order to implement this feature we will analyze techniques related to automated planning (Ghallab et al., 2004), to explore the graph of service in search for relevant compositions.

\section{Code generator}

Expected future functionality for this module include:

- More target platforms - It consists in implementing the code generation for other other programming languages and hardware platforms.

- Two-way edition - Currently the code generation works in one-way, i.e. semantic description to code, though, if the developer changes the generated code, those changes will not be automatically reflected in the original service description. The purpose of this functionality is to synchronize changes in service description and generated code.

- Human-readable documentation generation - Similar to other tools, such as Swagger $^{1}$, we aim to generate documentation of the REST service for human consumption, using our service description model.

\section{Graphical user interface}

We consider the creation of a graphical user interface (GUI) or an plugin for an existing integrated development environment (IDE) that permits the developer to easily navigate through the graph of existing ontology concepts. In the case of the IDE, an autocompletion tool would contribute to an agile application development.

\footnotetext{
${ }^{1}$ https://swagger.io
} 


\section{Distributed semantic discovery}

In order to reflect more the principles of the Swarm platform, we aim to create a distributed semantic discovery mechanism, which include decentralized storage and reasoning. One possible strategy to accomplish this is to include advances in Linked Data Fragments (Verborgh et al., 2014, 2016), which consists in distributing the workload of a query between different servers. 


\section{Appendix A: Generated query}

1 PREFIX rdf: <http://www. w3.org/1999/02/22-rdf-syntax-ns\#>

2 PREFIX rdfs: <http://www.w3.org/2000/01/rdf-schema\#>

3 PREFIX swarm: <http://iotswarm. info/ontology\#>

4 PREFIX schema: <http://schema.org/>

5 PREFIX hydra: < http://www.w3.org/ns/hydra/core\#>

6 PREFIX xsd: <http://www.w3.org/2001/XMLSchema\#>

7 SELECT ?service ?serviceType ?operationType ?operationDescription ?broker ?description ?entry ?method

8 ?expects ?returns ?country ?city ?state ?street ?postalCode ?streetNumber ?roomName

9 ? latitude ?longitude ?minPrice ?maxPrice ?trustThreshold ?walletId ?owner ?latitudeScore ?longitudeScore

10 ?serviceSubsumptionScore ?servicePluginScore ?serviceExactScore

11 ?operationSubsumptionScore ?operationPluginScore ?operationExactScore

12 ?returnsSubsumptionScore ?returnsPluginScore ?returnsExactScore

13 ?expectsSubsumptionScore ?expectsPluginScore ?expectsExactScore

15 WHERE \{

17 ?serviceType rdfs:subClassOf* swarm:SwarmService

18 ?service swarm:operations ?operation

19 ?service swarm:geolocation ?geolocation

?service swarm:broker ?broker

?service swarm:description ?description

OPTIONAL \{ ?service swarm:owner ?owner. \}

?operation swarm: entry ?entry

?operation rdf:type ?operationType

?operation swarm:method ?method

?operation swarm: returns ?returns

OPTIONAL \{ ?operation swarm:expects ?expects. \}

OPTIONAL \{ ?operation swarm:description ?operationDescription . \}

OPTIONAL \{ ?geolocation swarm: country ?country . \}

OPTIONAL \{ ?geolocation swarm: city ?city . \}

OPTIONAL \{ ?geolocation swarm: state ?state. \}

OPTIONAL \{ ?geolocation swarm:street ?street. \}

OPTIONAL \{ ?geolocation swarm:postalCode ?postalCode.

OPTIONAL \{?geolocation swarm:streetNumber ?streetNumber. \}

OPTIONAL \{ ?geolocation swarm:latitude ?latitude

?geolocation swarm:longitude ?longitude . \}

OPTIONAL \{ ?geolocation swarm:roomName ?roomName . \}

OPTIONAL \{ ?service swarm:pricing ?pricing . \}

OPTIONAL \{ ?pricing swarm:minPrice ?minPrice. \}

OPTIONAL \{ ?pricing swarm:maxPrice ?maxPrice . \}

OPTIONAL \{?pricing swarm:trustThreshold ?trustThreshold . \}

OPTIONAL \{ ?pricing swarm: walletId ?walletId . \}

OPTIONAL\{ swarm:SecurityCameraService rdfs:subClassOf+ ?serviceType

$\operatorname{BIND}(1$ as ?serviceSubsumptionScore) .

OPTIONAL\{ ? serviceType rdfs:subClassOf + swarm: SecurityCameraService $\operatorname{BIND}(3$ as ?servicePluginScore).

OPTIONAL $\{$ FILTER (? serviceType = swarm: SecurityCameraService $)$

$\operatorname{BIND}(5$ as ?serviceExactScore).

OPTIONAL \{ swarm:VisionDetectionOperation $\mathrm{rdfs}$ : subClassOf + ?operationType $\operatorname{BIND}(1$ as ?operationSubsumptionScore). 
OPTIONAL\{ ?operationType $\mathrm{rdfs}$ : subClassOf+ swarm:VisionDetectionOperation . $\operatorname{BIND}(3$ as ?operationPluginScore).

OPTIONAL \{ FILTER (?operationType = swarm: VisionDetectionOperation). $\operatorname{BIND}(5$ as ?operationExactScore).

OPTIONAL\{ swarm:StreetVideoObject rdfs:subClassOf+ ?expects.

BIND (3 as ?expectsSubsumptionScore). \}

OPTIONAL \{ ?expects rdfs : subClassof + swarm: StreetVideoObject $\operatorname{BIND}(1$ as ?expectsPluginScore). \}

OPTIONAL $\{$ FILTER $($ expects = swarm: StreetVideoObject $)$.

$\operatorname{BIND}(5$ as ?expectsExactScore).

OPTIONAL $\{$ swarm: CatImageObject $\mathrm{rdfs}$ : subClassOf+ ? returns. $\operatorname{BIND}(1$ as ?returnsSubsumptionScore). $\}$

OPTIONAL\{ ?returns rdfs: subClassOf + swarm: CatImageObject . $\operatorname{BIND}(3$ as ?returnsPluginScore).

OPTIONAL $\{$ FILTER ( returns $=$ swarm $:$ CatImageObject $)$. $\operatorname{BIND}(5$ as ?returnsExactScore).

FILTER (STR (? country) = "Argentina")

FILTER (STR(? city) = "Buenos Aires")

$\operatorname{FILTER}(\operatorname{STR}($ ? state $)=$ "BA")

FILTER(? minPrice $>=0$ )

FILTER $($ maxPrice $<=20$ )

FILTER (?operationSubsumptionScore $>0 \quad \|$ ?operationPluginScore || ?operationExactScore) .

FILTER (? serviceSubsumptionScore $>0 \quad||$ ?servicePluginScore || ?serviceExactScore) .

FILTER (? expectsSubsumptionScore $>0 \quad \|$ ? expectsPluginScore || ?expectsExactScore) .

FILTER(?returnsSubsumptionScore $>0 \quad||$ ?returnsPluginScore || ?returnsExactScore) . 


\section{Appendix B: Swarm ontology}

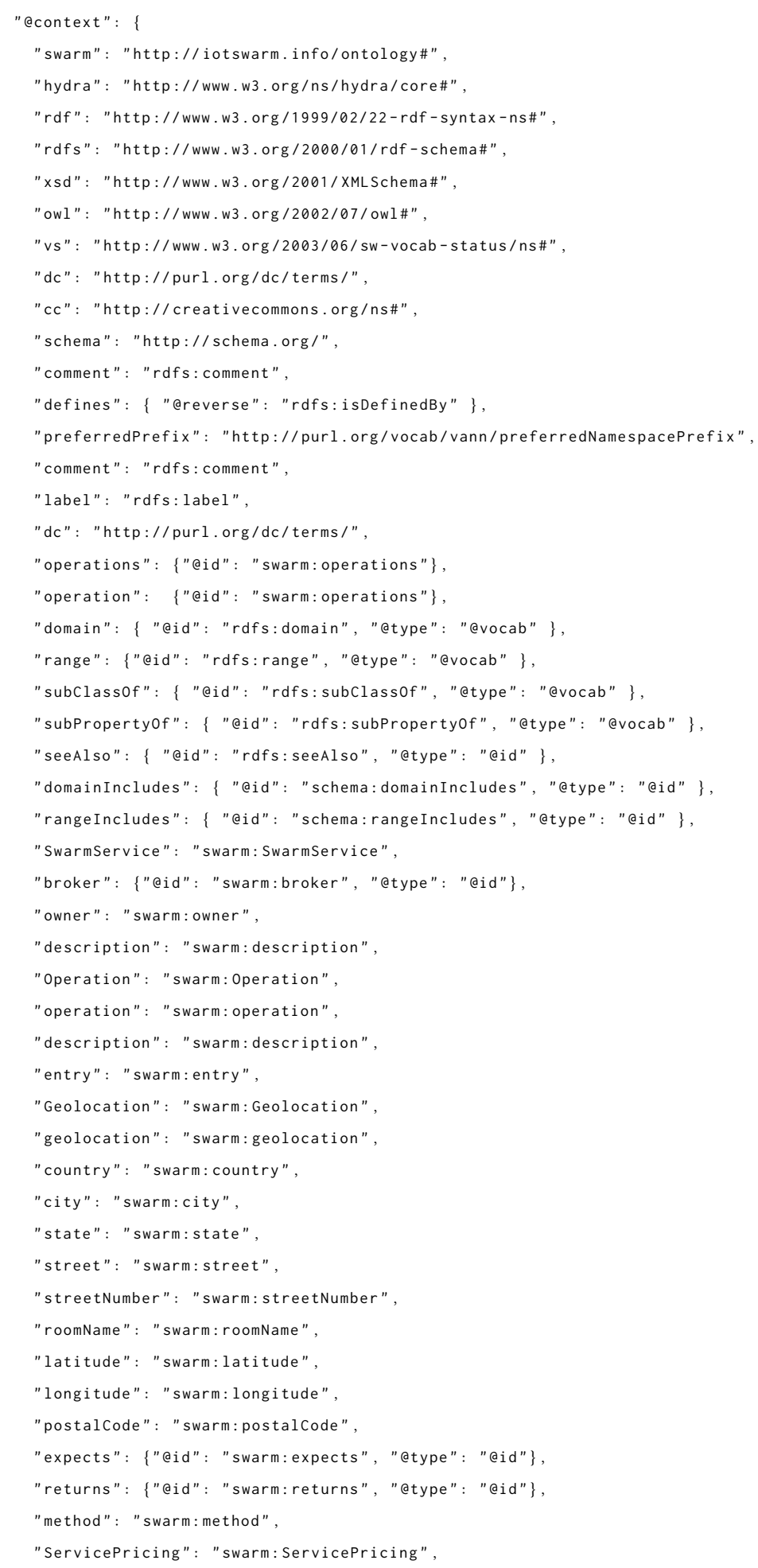




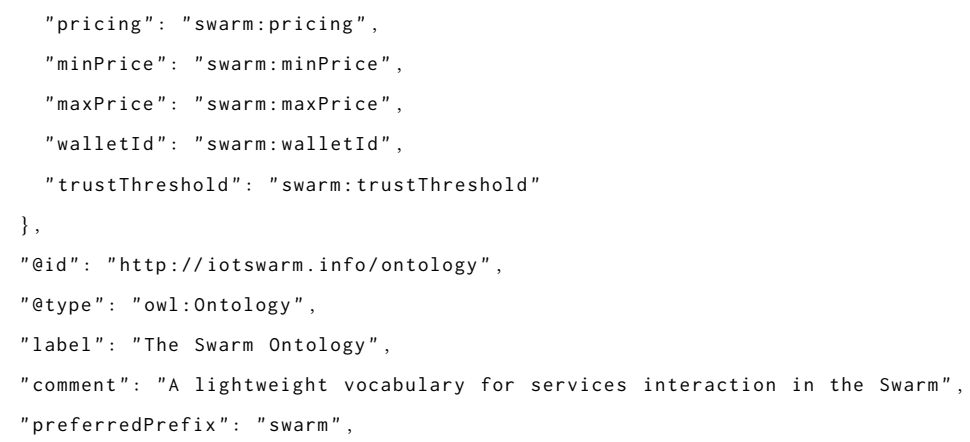


$111\}$,

112

113

(1)

"@id": "swarm: description",

"@type": "hydra:Property",

"label": "description",

"comment": "Human-friendly description of an operation or service",

"domainIncludes": ["swarm:Operation", "swarm: SwarmService"],

"range": "xsd:string"

\} ,

\{

"@id": "swarm: entry",

"@type": "rdf: Property",

"label": "entry",

"comment": "The path part of a URL",

"domain": "swarm: Operation",

"range": "xsd:string"

\} ,

\{

"@id": "swarm: Geolocation",

"@type": "rdfs: Class",

"label": "Geolocation",

"comment": "Service geolocation"

\} ,

\{

"@id": "swarm: geolocation",

"@type": "rdf: Property",

"label": "geolocation",

"comment": "The geolocation related to a Swarm service",

"domain": "swarm: SwarmService",

"range": "swarm:Geolocation"

\} ,

\{

"@id": "swarm: country",

"@type": "rdf: Property",

"subPropertyof": "schema: addresscountry",

"label": "country",

"comment": "The country where the service is physically running",

"domain": "swarm: Geolocation",

"range": "schema: Country"

\} ,

\{

"@id": "swarm:city",

"@type": "rdf: Property",

"subPropertyof": "schema: addressLocality",

"label": "city",

"comment": "The city where the service is physically running",

"domain": "swarm:Geolocation",

"range": "xsd:string"

\} ,

\{

"@id": "swarm:state",

"@type": "rdf:Property",

"subPropertyof": "schema: addressRegion",

"label": "state",

"comment": "The state where the service is physically running",

"domain": "swarm:Geolocation",

"range": "xsd:string"

\} ,

\{

"@id": "swarm:street",

"@type": "rdf:Property", 


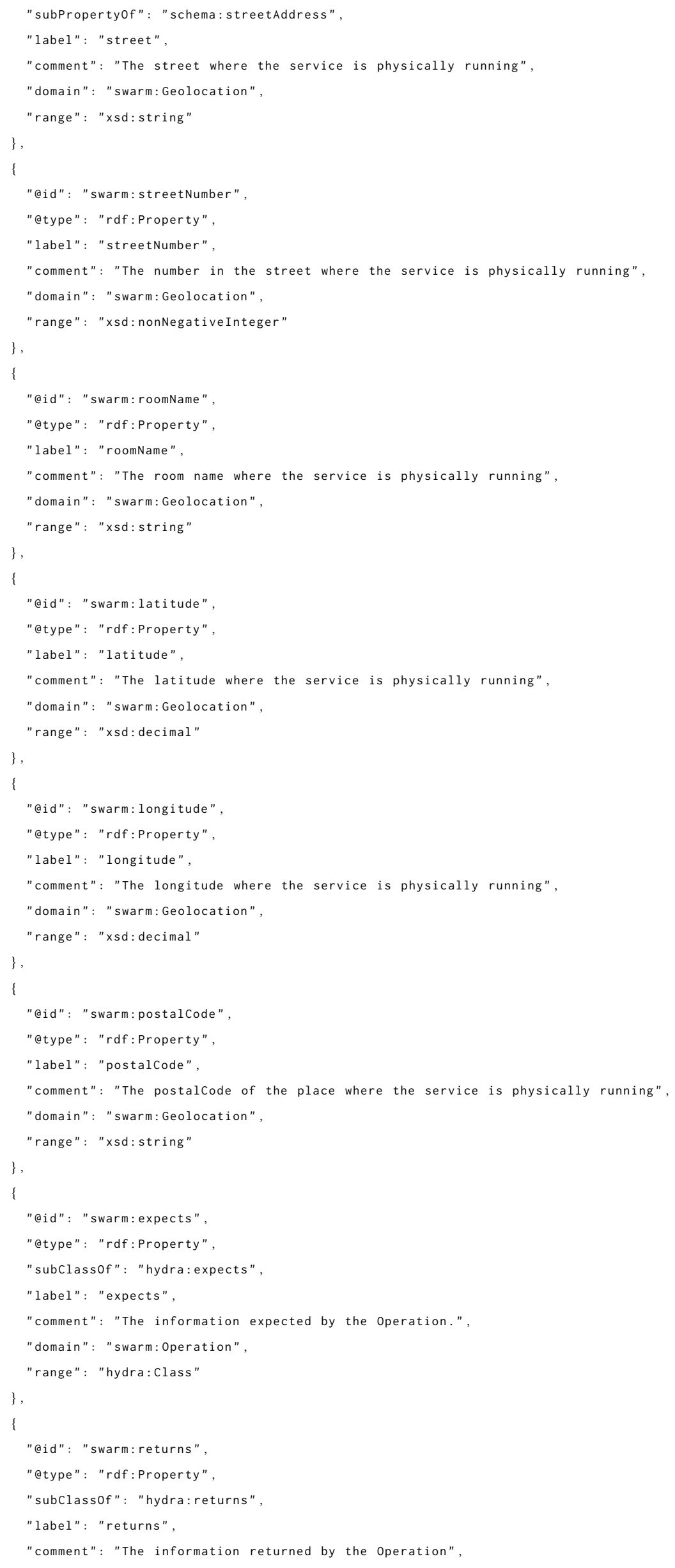




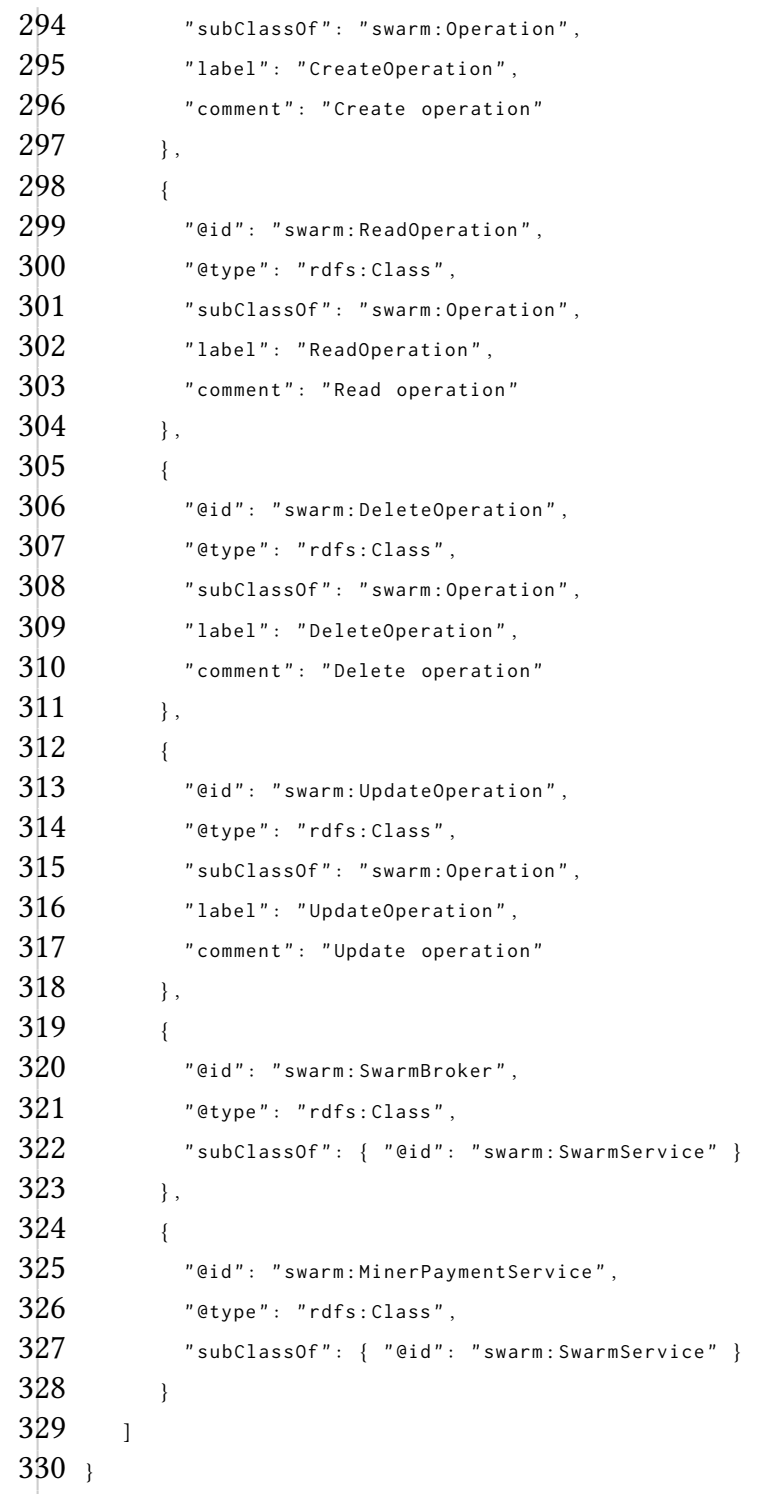




\section{Bibliography}

Aggarwal, C. C., Ashish, N., and Sheth, A. The Internet of Things: A Survey from the DataCentric Perspective. In Aggarwal, C. C., editor, Managing and Mining Sensor Data, pages 383428. Springer US, January 2013. ISBN 978-1-4614-6308-5 978-1-4614-6309-2. URL http://link. springer.com/chapter/10.1007/978-1-4614-6309-2_12.00058. 1

Alarcon, R., Wilde, E., and Bellido, J. Hypermedia-driven restful service composition. In Maximilien, E. M., Rossi, G., Yuan, S.-T., Ludwig, H., and Fantinato, M., editors, Service-Oriented Computing, pages 111-120, Berlin, Heidelberg, 2011. Springer Berlin Heidelberg. ISBN 978-3642-19394-1. 19, 65

Atzori, L., Iera, A., and Morabito, G. The Internet of Things: A survey. Computer Networks, 54 (15):2787-2805, October 2010. ISSN 1389-1286. doi: 10.1016/j.comnet.2010.05.010. URL http: //www.sciencedirect.com/science/article/pii/S1389128610001568. 02552. 1

Atzori, L., Iera, A., Morabito, G., and Nitti, M. The Social Internet of Things (SIoT) - When social networks meet the Internet of Things: Concept, architecture and network characterization. Computer Networks, 56(16):3594-3608, November 2012. ISSN 1389-1286. doi: 10.1016/j.comnet. 2012.07.010. URL http://www.sciencedirect.com/science/article/pii/S1389128612002654. 16

Bogliolo, A., Polidori, P., Aldini, A., Moreira, W., Mendes, P., Yildiz, M., Ballester, C., and Seigneur, J.-M. Virtual currency and reputation-based cooperation incentives in user-centric networks. In Wireless Communications and Mobile Computing Conference (IWCMC), 2012 8th International, pages 895-900. IEEE, 2012. 46

Boley, H. and Chang, E. Digital ecosystems: Principles and semantics. In Digital EcoSystems and Technologies Conference, 2007. DEST'07. Inaugural IEEE-IES, pages 398-403. IEEE, 2007. 15

Bonomi, F., Milito, R., Zhu, J., and Addepalli, S. Fog computing and its role in the internet of things. In Proceedings of the first edition of the MCC workshop on Mobile cloud computing, pages 13-16. ACM, 2012. 17

Calcina-Ccori, P., Costa, L., Fedrecheski, G., Esquiagola, J., Zuffo, M., and da Silva, F. C. Agile servient integration with the swarm: Automatic code generation for nodes in the internet of things. In Proceedings of the International Conference on Future Networks and Distributed Systems, page 22. ACM, 2017. 47, 70

Calcina-Ccori, P. C., De Biase, L. C. C., Fedrecheski, G., da Silva, F. S. C., and Zuffo, M. K. Enabling semantic discovery in the swarm. IEEE Transactions on Consumer Electronics, 65(1):57-63, 2018. 39,70

Calcina-Ccori, P. C., De Biase, L. C., De Oliveira, C. E. L., Fedrecheski, G., da Silva, F. C., and Zuffo, M. K. Describing services geolocation in iot context. In 2019 IEEE International Conference on Consumer Electronics (ICCE), pages 1-2. IEEE, 2019a. 45, 70 
Calcina-Ccori, P. C., De Biase, L. C., De Oliveira, C. E. L., Fedrecheski, G., da Silva, F. C., and Zuffo, M. K. Location-aware discovery of services in the iot: a swarm approach. In 2019 Global IoT Summit (GIoTS), pages 1-6. IEEE, 2019b. 45, 70

Calcina-Ccori, P. C., De Biase, L. C., Fedrecheski, G., da Silva, F. C., and Zuffo, M. K. SwarmGen: a framework for automatic generation of semantic services in an IoT network. In 2020 IEEE International Conference on Consumer Electronics (ICCE). IEEE, 2020. 48, 70

Costa, L., Calcina, P., Rabaey, J., Zuffo, M., and Wolisz, A. Semantic Swarm. In Second International Workshop on the Swarm at the Edge of the Cloud (Article In Press), Seattle, USA, 2015a. 44, 70

Costa, L., Rabaey, J., Wolisz, A., Calixto, G., and Zuffo, M. Swarm OS control plane: An architecture proposal for heterogeneous and organic networks. In 2015 IEEE International Conference on Consumer Electronics (ICCE), pages 245-246, January 2015b. doi: 10.1109/ICCE.2015.7066398. 2,7

De Biase, C. L., Ccori, C. P., Fedrecheski, G., Navarro, D., Lino, R. Y., and Zuffo, M. K. Swarm minimum broker: an approach to deal with the internet of things heterogeneity. In 2018 Global Internet of Things Summit (GIoTS), pages 1-6. IEEE, 2018a. 13

De Biase, L. C., Calcina-Ccori, P. C., Silva, F. S., and Zuffo, M. K. The semantic mediation for the swarm: An adaptable and organic solution for the internet of things. In 2017 IEEE International Conference on Consumer Electronics (ICCE), pages 78-79. IEEE, 2017. 70

De Biase, L. C., Calcina-Ccori, P. C., Fedrecheski, G., Duarte, G. M., Rangel, P. S., and Zuffo, M. K. Swarm economy: a model for transactions in a distributed and organic iot platform. IEEE Internet of Things fournal, 2018b. 11, 12, 46, 54, 70

De Biase, L. C., Fedrecheski, G., Calcina-Ccori, P. C., De Deus Lopes, R., and K., Z. M. Surveillance Swarm: Gathering Resources for Finding Targets in a Distributed Global Network of Smart Devices. In 2020 IEEE International Conference on Consumer Electronics (ICCE). IEEE, 2020. 55, 70

Esquiagola, J., Costa, L., Calcina, P., and Zuffo, M. Enabling coap into the swarm: A transparent interception coap-http proxy for the internet of things. In Global Internet of Things Summit (GIoTS), 2017, pages 1-6. IEEE, 2017. 12

Farnaghi, M. and Mansourian, A. Automatic composition of wsmo based geospatial semantic web services using artificial intelligence planning. Journal of Spatial Science, 58(2):235-250, 2013. 67

Fedrecheski, G., De Biase, L. C. C., Calcina-Ccori, P. C., and Zuffo, M. K. Attribute-based access control for the swarm with distributed policy management. IEEE Transactions on Consumer Electronics, 65(1):90-98, 2018. 13, 29, 54

Fielding, R. T. and Taylor, R. N. Principled Design of the Modern Web Architecture. ACM Trans. Internet Technol., 2(2):115-150, May 2002. ISSN 1533-5399. doi: 10.1145/514183.514185. URL http://doi.acm.org/10.1145/514183.514185. 01687. 21, 30, 38, 66

Fowler, M. and Lewis, J. Microservices a definition of this new architectural term, 2014. URL http://martinfowler.com/articles/microservices.html. 8

Gamma, E., Helm, R., Johnson, R., and Vlissides, J. Design patterns: elements of reusable objectoriented software. Addison-Wesley Professional, 1995. ISBN 0201633612. 23, 25 
Ghallab, M., Nau, D., and Traverso, P. Automated Planning: theory and practice. Elsevier, 2004. 71

Giang, N. K., Blackstock, M., Lea, R., and Leung, V. C. Developing iot applications in the fog: A distributed dataflow approach. In 2015 5th International Conference on the Internet of Things (IOT), pages 155-162. IEEE, 2015. 3

Giusto, D., Iera, A., Morabito, G., and Atzori, L. The internet of things: 20th Tyrrhenian workshop on digital communications. Springer Science \& Business Media, 2010. 1

Hillar, G. C. Internet of Things with Python. Packt Publishing Ltd, 2016. 53

Horn, P. Autonomic computing: IBM's Perspective on the State of Information Technology. Technical report, 2001. 15

Hu, V., Ferraiolo, D., Kuhn, D., Schnitzer, A., Sandlin, K., Miller, R., and Scarfone, K. Guide to attribute based access control (abac) definition and considerations. Technical report, National Institute of Standards and Technology, 012014.14

Hu, V. C., Kuhn, D. R., Ferraiolo, D. F., and Voas, J. Attribute-based access control. Computer, 48 (2):85-88, 2015. 13

Jos de Bruijn, Christoph Bussler, John Domingue, Dieter Fensel, and Martin Hepp. Web Service Modeling Ontology (WSMO). Technical report, 2005. URL https://www.w3.org/Submission/ $\mathrm{WSMO} / .17$

Klusch, M., Gerber, A., and Schmidt, M. Semantic web service composition planning with owlsxplan. In AAAI Fall Symposium: Agents and the Semantic Web, pages 55-62, 2005. 17, 67

Kolbe, N., Kubler, S., Robert, J., Le Traon, Y., and Zaslavsky, A. Linked vocabulary recommendation tools for internet of things: a survey. ACM Computing Surveys (CSUR), 51(6):1-31, 2019. 5

Kopeckỳ, J., Gomadam, K., and Vitvar, T. hrests: An html microformat for describing restful web services. In 2008 IEEE/WIC/ACM International Conference on Web Intelligence and Intelligent Agent Technology, volume 1, pages 619-625. IEEE, 2008. 18

Lanthaler, M. and Gutl, C. A semantic description language for RESTful Data Services to combat Semaphobia. In 2011 Proceedings of the 5th IEEE International Conference on Digital Ecosystems and Technologies Conference (DEST), pages 47-53, May 2011. doi: 10.1109/DEST.2011.5936597. 34

Lanthaler, M. Creating 3rd generation web apis with hydra. In Proceedings of the 22Nd international conference on world wide web, pages 35-38, 2013. 18, 30, 32, 66

Lanthaler, M. Third Generation Web APIs. PhD thesis, Graz University of Technology, Austria, 2014. 36

Lee, E. A., Rabaey, J., Hartmann, B., Kubiatowicz, J., Pister, K., Sangiovanni-Vincentelli, A., Seshia, S. A., Wawrzynek, J., Wessel, D., Rosing, T. S., Blaauw, D., Dutta, P., Fu, K., Guestrin, C., Taskar, B., Jafari, R., Jones, D., Kumar, V., Mangharam, R., Pappas, G. J., Murray, R. M., and Rowe, A. The Swarm at the Edge of the Cloud. IEEE Design \& Test, 31(3):8-20, June 2014. ISSN 21682356, 2168-2364. doi: 10.1109/MDAT.2014.2314600. URL http://ieeexplore.ieee.org/document/ $6781658 / .2,7$ 
Li, L. and Horrocks, I. A software framework for matchmaking based on semantic web technology. International fournal of Electronic Commerce, 8(4):39-60, 2004. doi: 10.1080/10864415. 2004.11044307. URL https://doi.org/10.1080/10864415.2004.11044307. 44

Lokhande, P., Aslam, F., Hawa, N., Munir, J., and Gulamgaus, M. Efficient way of web development using python and flask. International fournal of Advanced Research in Computer Science, 2015. 53

Madisetti, V. and Bahga, A. Internet of Things. VPT, 1st edition, 2014. ISBN 978-0-9960255-1-5. 53

Martin, D., Burstein, M., Hobbs, J., Lassila, O., McDermott, D., McIlraith, S., Narayanan, S., Paolucci, M., Parsia, B., Payne, T., Sirin, E., Srinivasan, N., and Sycara, K. OWL-S: Semantic Markup for Web Services, 2004. URL http://www.w3.org/Submission/OWL-S/. 17

Mayer, S., Hodges, J., Yu, D., Kritzler, M., and Michahelles, F. An open semantic framework for the industrial internet of things. IEEE Intelligent Systems, 32(1):96-101, 2017. 19, 65

Noura, M., Atiquzzaman, M., and Gaedke, M. Interoperability in internet of things: Taxonomies and open challenges. Mobile Networks and Applications, 24(3):796-809, 2019. 5

O'Sullivan, J., Edmond, D., and ter Hofstede, A. What's in a Service? Distributed and Parallel Databases, 12(2):117-133, September 2002. ISSN 1573-7578. doi: 10.1023/A:1016547000822. URL https://doi.org/10.1023/A:1016547000822. 29

O’Sullivan, J., Edmond, D., and ter Hofstede, A. H. Formal description of non-functional service properties. Technical report, Centre for Information Technology, Queensland University of Technology, 2005. xv, 29, 30

Rabaey, J. The swarm at the edge of the cloud - A new perspective on wireless. In 2011 Symposium on VLSI Circuits (VLSIC), pages 6-8, June 2011. 7

Sethi, P. and Sarangi, S. R. Internet of Things: Architectures, Protocols, and Applications. Journal of Electrical and Computer Engineering, 2017:1-25, 2017. ISSN 2090-0147, 2090-0155. doi: 10. 1155/2017/9324035. URL https://www.hindawi.com/journals/jece/2017/9324035/. 1

Shi, W., Cao, J., Zhang, Q., Li, Y., and Xu, L. Edge computing: Vision and challenges. IEEE Internet of Things fournal, 3(5):637-646, 2016. 17

Silva, A. L. M., Pérez-Alcázar, J. d. J., and Kofuji, S. T. Interoperability in semantic web of things: Design issues and solutions. International fournal of Communication Systems, 32(6):e3911, 2019. 18,65

Sirin, E., Parsia, B., Wu, D., Hendler, J., and Nau, D. Htn planning for web service composition using shop2. Journal of Web Semantics, 1(4):377-396, 2004. 17, 67

Siviero, A., Bisht, A., MacGillivray, C., and Torchia, M. Worldwide internet of things spending guide. Technical report, International Data Corporation (IDC), 07 2019. URL https://www.idc. com/getfile.dyn?containerId=IDC_P29475\&attachmentId=47356047. 1

Taivalsaari, A. and Mikkonen, T. A roadmap to the programmable world: Software challenges in the iot era. IEEE Software, 34(1):72-80, Jan 2017. ISSN 1937-4194. doi: 10.1109/MS.2017.26. 1

Tsiatsis, V., Karnouskos, S., Holler, J., Boyle, D., and Mulligan, C. Internet of Things: Technologies and Applications for a New Age of Intelligence. Academic Press, 2 edition, 2019. 2 
UN HABITAT. Urbanization and Development. Emerging Futures. Technical report, United Nations, 2016. 2

Vaculín, R. and Sycara, K. Towards automatic mediation of owl-s process models. In IEEE International Conference on Web Services (ICWS 2007), pages 1032-1039. IEEE, 2007. 17, 67

Verborgh, R., Vander Sande, M., Colpaert, P., Coppens, S., Mannens, E., and Van de Walle, R. Web-scale querying through linked data fragments. In LDOW. Citeseer, 2014. 72

Verborgh, R., Vander Sande, M., Hartig, O., Van Herwegen, J., De Vocht, L., De Meester, B., Haesendonck, G., and Colpaert, P. Triple pattern fragments: a low-cost knowledge graph interface for the web. Journal of Web Semantics, 37:184-206, 2016. 72

Vitvar, T., Kopeckỳ, J., Viskova, J., and Fensel, D. Wsmo-lite annotations for web services. In European Semantic Web Conference, pages 674-689. Springer, 2008. 18

Von Rosing, M., Laurier, W., and Polovina, S. M. The Value of Ontology. In The complete business process handbook: body of knowledge from process modeling to BPM, volume 1, pages 91-199. Morgan Kaufmann, 2014. 39

Weiser, M. Human-computer interaction. chapter The Computer for the 21st Century, pages 933-940. Morgan Kaufmann Publishers Inc., San Francisco, CA, USA, 1995. ISBN 1-55860-2461. URL http://dl.acm.org/citation.cfm?id=212925.213017. 15

Zambonelli, F. Toward Sociotechnical Urban Superorganisms. Computer, 45(8):76-78, August 2012. ISSN 0018-9162. doi: 10.1109/MC.2012.280. 16

Zorgati, H., Djemaa, R. B., and Amor, I. A. B. Service discovery techniques in internet of things: a survey. In 2019 IEEE International Conference on Systems, Man and Cybernetics (SMC), pages 1720-1725. IEEE, 2019. 5 